\title{
The Direct Rh(III)-Catalyzed C-H Amidation of Aniline Derivatives Using a Pyrimidine Directing Group: The Selective Solvent Controlled Synthesis of 1,2- Diaminobenzenes and Benzimidazoles
}

\author{
Shrikant M. Khake and Naoto Chatani* \\ Department of Applied Chemistry, Faculty of Engineering, Suita, Osaka-565-0871, Japan \\ chatani@chem.eng.osaka-u.ac.jp
}

1 General Information $\quad$ S3

2 Materials $\quad$ S3

$3 \quad$ Synthesis of Starting Materials $\quad$ S3

3.1 General Procedure for the Preparation of $N$-Phenylpyrimidin-2-amine S3 Derivatives

$\begin{array}{ll}\text { 3.2 Procedure for the Synthesis of }[\mathrm{D}]-\mathbf{1 a} & \text { S7 }\end{array}$

4 Optimization of the Reactions Conditions $\quad$ S9

5 General Procedure for the Rh-Catalyzed C-H Amidation $\quad$ S10

Procedure for Gram Scale Synthesis of 3aa $\quad$ S11

6 Characterization Data of Amidated Compounds S11

$\begin{array}{ll}\text { Characterization Data of Compounds } 3 & \text { S11 }\end{array}$

7 General Procedure for the Rh-Catalyzed $\mathrm{C}-\mathrm{H}$ Amidation followed S27 Cyclization

$\begin{array}{lr}\text { Characterization Data of Compounds } 5 & \text { S28 }\end{array}$

8 General Procedure for the Rh-Catalyzed C-H Diamidation followed S32 Cyclization

8.1 Characterization Data of Cyclized Product $6 \quad$ S33

$\begin{array}{ll}\text { 8.2 Characterization Data for Cyclized Product } 7 & \text { S35 }\end{array}$ 
9 Chemoselective Deprotection $\quad$ S36

9.1 Deprotection of Amide Group of 3aa to Form 8

9.2 Deprotection of Pyrimidinyl Group of 5 to Form 9

9.3 Deprotection of Amide as well as Pyrimidinyl Group of $\mathbf{6 b k}$ to Form S38

10

10 Control Experiments $\quad$ S39

$\begin{array}{ll}\text { 10.1 Procedure for Reaction of } \mathbf{5 b j} \text { with } \mathbf{2} \mathbf{j} & \text { S39 }\end{array}$

$\begin{array}{lr}\text { 10.2 External Additive Experiments } & \text { S39 }\end{array}$

$\begin{array}{lr}10.3 \text { Competition Experiment } & \text { S39 }\end{array}$

10.4 Procedure for Kinetic Isotope Effect Experiment $\quad$ S40

10.5 Procedure for H/D Scrambling Experiment $\quad$ S41

11 X-ray Crystallographic Data $\quad$ S43

12 References $\quad$ S61

$13{ }^{1} \mathrm{H}$ and ${ }^{13} \mathrm{C}$ NMR Spectra of Compounds

13.1 Spectra of Compounds 1 $\quad$ S62

13.2 Spectra of Compounds $3 \quad$ S68

13.3 Spectra of Compounds $5 \quad$ S96

$\begin{array}{lr}\text { 13.4 Spectra of Compound } 6 & \text { S105 }\end{array}$

$\begin{array}{lr}\text { 13.5 Spectra of Compound } 7 & \text { S109 }\end{array}$

$\begin{array}{lr}\text { 13.6 Spectra of Compound } 8 & \text { S110 }\end{array}$

13.7 Spectra of Compound $9 \quad$ S111

$\begin{array}{lr}\text { 13.8 Spectra of Compound } \mathbf{1 0} & \text { S114 }\end{array}$ 
1. General Information: ${ }^{1} \mathrm{H}$ NMR and ${ }^{13} \mathrm{C}$ NMR spectra were recorded on a JEOL ECS-400 spectrometeror VARIAN UNITY INOVA-600 spectrometer in $\mathrm{CDCl}_{3}$. All the data are reported as we see follow: chemical shift $(\delta)$ peaking in ppm, multiplicity $(\mathrm{s}=$ singlet, $\mathrm{d}=$ doublet, $\mathrm{t}=$ triplet, $\mathrm{q}=$ quartet, quin = quintet, $\mathrm{sx}=$ sextuplet and $\mathrm{m}=$ multiple), coupling constant $(J)$ in Hz, and integration. Infrared spectra (IR) were obtained using a JASCO FT/IR4200 spectrometer; absorptions are reported in reciprocal centimetres with the following relative intensities: $\mathrm{s}$ (strong), $\mathrm{m}$ (medium), or w (weak). Mass spectra and high resolution mass spectra (HRMS) were obtained using a JEOL JMS-700 spectrometer with a time-of-flight mass analyzer. Melting points were determined using a Opti Melt MPA 100 Automated melting point system (temperature measurement accuracy is $0.1^{\circ} \mathrm{C}$ ). Column chromatography was performed with $\mathrm{SiO}_{2}$ (Silicycle Silica Flash F60 (230-400 mesh)). Also, we proceed the isolation for some product using there is compound was purified by LC-908HPLC(GPC).

2. Materials: $\left[\mathrm{Cp}^{*} \mathrm{RhCl}_{2}\right]_{2}(\mathrm{CAS} 12354-85-7)$ was purchased from Sigma Aldrich. AgSbF 6 (CAS 26042-64-8) was purchased from Tokyo Chemical Industry Co., Ltd. Hexafluoroisopropanol (HFIP) (CAS 920-66-1) and trifluoroethanol (CAS 75-89-8) were purchased from Tokyo Chemical Industry Co., Ltd.

\section{Synthesis of Starting Materials:}

$N$-Phenylpyrimidin-2-amine derivatives (1a-1h), as well as the dioxozolone derivatives (2a, $\mathbf{2 b}, \mathbf{2 d}, \mathbf{2 e}, \mathbf{2 j}){ }^{2}(\mathbf{2 c}, \mathbf{2 f}, \mathbf{2 g}, \mathbf{2 h}),{ }^{3}(\mathbf{2 i}, \mathbf{2 k}, \mathbf{2 l}, \mathbf{2 m}, \mathbf{2 o})^{4}$ and $\mathbf{1} \mathbf{n}^{5}$ were synthesized by previously reported procedures. ${ }^{1}$

\subsection{General Procedure for the Preparation of $N$-Phenylpyrimidin-2-amine Derivatives: ${ }^{1}$}

$N$-Phenylpyrimidin-2-amine derivatives (1i-1n) were synthesized following a procedure similar to that used for the synthesis of $N$-(o-tolyl)pyrimidin-2-amine (1a). ${ }^{1}$ To a stirred solution of 2-chloropyrimidine $(2.5 \mathrm{~g}, 21.9 \mathrm{mmol})$ and 4-methoxy-2-methylaniline (3.0 g, 21.9 $\mathrm{mmol})$ in $80 \mathrm{~mL}$ of $\mathrm{EtOH} / \mathrm{H}_{2} \mathrm{O}(4 / 1)$ was added conc. $\mathrm{HCl}(2.2 \mathrm{~mL})$, and resulting reaction mixture was stirred for $8 \mathrm{~h}$ at $100{ }^{\circ} \mathrm{C}$ in preheated oil bath. The reaction mixture was then allowed to cool to room temperature, and the solvent was evaporated under reduced pressure. The residue was poured into $\mathrm{H}_{2} \mathrm{O}(200 \mathrm{~mL})$ and the resulting solution extracted with EtOAc (4 x $100 \mathrm{~mL}$ ). The combined organic layers were dried over $\mathrm{MgSO}_{4}$. After filtration and evaporation of the solvent under reduced pressure, the crude product was purified by column 
chromatography on silica gel (eluent: petroleum ether/EtOAc=1/1) to give $\mathbf{1 i}(1.5 \mathrm{~g}, 32 \%)$ as a light yellow solid.<smiles>COc1ccc(Nc2ncccn2)c(C)c1</smiles>

\section{$N$-(4-methoxy-2-methylphenyl)pyrimidin-2-amine (1i)}

$32 \%$ yield. (eluent: petroleum ether $/$ EtOAc $=1 / 1, R_{f}=0.342$ ). Light yellow solid.

$\mathrm{Mp}=114-115^{\circ} \mathrm{C}$.

${ }^{1} \mathbf{H}$ NMR $\left(\mathrm{CDCl}_{3}, 400 \mathrm{MHz}\right): \delta 8.34(\mathrm{~d}, \mathrm{~J}=4.8 \mathrm{~Hz}, 2 \mathrm{H}), 7.51(\mathrm{~d}, \mathrm{~J}=8.2 \mathrm{~Hz}, 1 \mathrm{H}), 6.80-6.77(\mathrm{~m}$, $3 \mathrm{H}), 6.62(\mathrm{t}, \mathrm{J}=4.8 \mathrm{~Hz}, 1 \mathrm{H}), 3.80(\mathrm{~s}, 3 \mathrm{H}), 2.27$ (s, 3H).

${ }^{13} \mathrm{C}$ NMR $\left(\mathrm{CDCl}_{3}, 100 \mathrm{MHz}\right): \delta 161.6,158.3,157.2,134.0,130.1,126.3,116.2,111.8,55.5$, 18.4 .

IR (neat) cm $\mathrm{cm}^{-1}: 3238 \mathrm{w}, 3002 \mathrm{w}, 2968 \mathrm{w}, 2951 \mathrm{w}, 2834 \mathrm{w}, 2361 \mathrm{~s}, 2339 \mathrm{~s}, 1583 \mathrm{~s}, 1520 \mathrm{~s}, 1501$ s, 1446 s, 1405 s, 1374 w, 1284 m, 1260 m, 1225 s, 1159 m, 1118 w, 1046 m, 991 w, 850 w, $799 \mathrm{~m}, 714 \mathrm{w}, 667 \mathrm{w}, 656 \mathrm{w}$.

HRMS (EI) m/z: [M] $]^{+}$Calcd for $\mathrm{C}_{12} \mathrm{H}_{13} \mathrm{~N}_{3} \mathrm{O}$ : 215.1059; Found: 215.1062 .<smiles>Cc1ccc(Nc2ncccn2)cc1</smiles>

\section{$N$-(4-tolyl)pyrimidin-2-amine (1j)}

This compound was prepared by reacting 2-chloropyrimidine (3.2 g, $28.0 \mathrm{mmol})$, p-toluidine (3 g, $28.0 \mathrm{mmol})$ and conc. $\mathrm{HCl}(2.8 \mathrm{~mL})$. Purification by column chromatography on silica gel (eluent: petroleum ether/EtOAc $\left.=1 / 1, \mathrm{R}_{\mathrm{f}}=0.46\right)$ gave $\mathbf{1 j}(2.23 \mathrm{~g}, 43 \%)$ as a yellow solid. $\mathrm{Mp}$ $=131-132{ }^{\circ} \mathrm{C}$.

${ }^{1} \mathbf{H}$ NMR $\left(\mathrm{CDCl}_{3}, 400 \mathrm{MHz}\right): \delta 8.40(\mathrm{~d}, \mathrm{~J}=4.8 \mathrm{~Hz}, 2 \mathrm{H}), 7.49-7.46(\mathrm{~m}, 3 \mathrm{H}), 7.15(\mathrm{~d}, \mathrm{~J}=8.2 \mathrm{~Hz}$, $2 \mathrm{H}), 6.67(\mathrm{t}, \mathrm{J}=4.8 \mathrm{~Hz}, 1 \mathrm{H}), 2.33(\mathrm{~s}, 3 \mathrm{H})$.

${ }^{13} \mathrm{C}$ NMR $\left(\mathrm{CDCl}_{3}, 100 \mathrm{MHz}\right): \delta 160.6,158.1,136.8,132.6,129.6,120.2,112.3,20.9$. 
IR (neat) cm $\mathrm{cm}^{-1}: 3259$ w, $3173 \mathrm{w}, 3100 \mathrm{w}, 3056 \mathrm{w}, 2970 \mathrm{w}, 2861 \mathrm{w}, 2361 \mathrm{~s}, 2339 \mathrm{~m}, 1614 \mathrm{~m}$, 1584 s, 1574 s, 1511 s, 1450 s, 1421 s, 1364 m, 1313 w, 1293 w, 1252 m, 1228 m, 1215 m, 1177 w, 1157 w, 1112 w, 1082 w, 996 m, 847 w, 815 m, 793 m, 781 m, 672 w.

HRMS (EI) m/z: [M] $]^{+}$Calcd for $\mathrm{C}_{11} \mathrm{H}_{11} \mathrm{~N}_{3}$ : 185.0953; Found: 185.0953.<smiles>COc1ccc(Nc2ncccn2)cc1</smiles>

\section{$N$-(4-methoxyphenyl)pyrimidin-2-amine (1k)}

This compound was prepared by reacting 2-chloropyrimidine (2.8 g, $24.4 \mathrm{mmol})$, 4methoxyaniline (3 g, $24.4 \mathrm{mmol})$ and conc. $\mathrm{HCl}(2.4 \mathrm{~mL})$. Purification by column chromatography on silica gel (eluent: petroleum ether $\left./ \mathrm{EtOAc}=2 / 1, \mathrm{R}_{\mathrm{f}}=0.36\right)$ gave $1 \mathrm{k}(1.5 \mathrm{~g}$, $30 \%)$ as a light brown solid. $\mathrm{Mp}=129-130^{\circ} \mathrm{C}$.

${ }^{1} \mathbf{H}$ NMR $\left(\mathrm{CDCl}_{3}, 400 \mathrm{MHz}\right): \delta 8.37(\mathrm{~d}, \mathrm{~J}=4.8 \mathrm{~Hz}, 2 \mathrm{H}), 7.47(\mathrm{td}, \mathrm{J}=9.0,3.4 \mathrm{~Hz}, 2 \mathrm{H}), 7.24$ (brs, 1H), $6.91(\mathrm{dt}, \mathrm{J}=9.0,3.4 \mathrm{~Hz}, 2 \mathrm{H}), 6.66(\mathrm{t}, \mathrm{J}=4.8 \mathrm{~Hz}, 1 \mathrm{H}), 3.80(\mathrm{~s}, 3 \mathrm{H})$.

${ }^{13} \mathbf{C} \mathbf{N M R}\left(\mathrm{CDCl}_{3}, 100 \mathrm{MHz}\right): \delta 160.8,158.2,155.9,132.4,122.4,114.4,112.1,55.6$.

IR (neat) cm $\mathrm{cm}^{-1}: 3262$ w, 3198 w, 3170 w, 3009 w, 2969 w, 2835 w, 2360 s, 1739 m, 1610 m, 1582 s, 1533 s, 1508 s, 1449 s, 1423 s, 1366 m, 1297 w, 1237 s, 1179 w, 1109 w, 1031 m, 825 m, $997 \mathrm{~m}, 730 \mathrm{w}, 671 \mathrm{w}$.

HRMS (EI) m/z: [M] $]^{+}$Calcd for $\mathrm{C}_{11} \mathrm{H}_{11} \mathrm{~N}_{3} \mathrm{O}$ : 201.0902; Found: 201.0901 .<smiles>Cc1cccc(Nc2ncccn2)c1</smiles>

\section{$N$-(m-tolyl)pyrimidin-2-amine (11)}

This compound was prepared by reacting 2-chloropyrimidine (3.2 g, $28.0 \mathrm{mmol}), \mathrm{m}$-toluidine $(3 \mathrm{~g}, 28.0 \mathrm{mmol})$ and conc. $\mathrm{HCl}(2.8 \mathrm{~mL})$. Purification by column chromatography on silica gel (eluent: petroleum ether/EtOAc=2/1, $\left.\mathrm{R}_{\mathrm{f}}=0.42\right)$ gave $\mathbf{1 l}(2.5 \mathrm{~g}, 48 \%)$ as a yellow solid. Mp $=97-98^{\circ} \mathrm{C}$.

${ }^{1} \mathbf{H}$ NMR $\left(\mathrm{CDCl}_{3}, 400 \mathrm{MHz}\right): \delta 8.41(\mathrm{~d}, \mathrm{~J}=4.9 \mathrm{~Hz}, 2 \mathrm{H}), 7.60$ (brs, 1H), 7.44-7.42 (m, 2H), $7.22(\mathrm{td}, \mathrm{J}=7.6,1.1 \mathrm{~Hz}, 1 \mathrm{H}), 6.88-6.66(\mathrm{~m}, 1 \mathrm{H}), 6.67(\mathrm{t}, \mathrm{J}=4.8 \mathrm{~Hz}, 1 \mathrm{H}), 2.36(\mathrm{~s}, 3 \mathrm{H})$. 
${ }^{13} \mathbf{C ~ N M R}\left(\mathrm{CDCl}_{3}, 100 \mathrm{MHz}\right): \delta 160.4,158.1,139.4,128.9,123.8,120.4,116.9,112.4,21.7$.

IR (neat) cm $\mathrm{cm}^{-1}: 3272$ w, 3179 w, 3107 w, 3203 w, 2970 w, 2946 w, 2863 w, 2360 s, 2339 m, 1739 m, 1578 s, 1535 s, 1490m, 1444 s, 1362 w, 1306 w, 1228 w, 1215 w, 1167 w, 992 w, 889 w, $865 \mathrm{w}, 796 \mathrm{~m}, 777 \mathrm{~m}, 749 \mathrm{w}, 694 \mathrm{~m}, 671 \mathrm{w}$.

HRMS (EI) m/z: [M] $]^{+}$Calcd for $\mathrm{C}_{11} \mathrm{H}_{11} \mathrm{~N}_{3}$ : 185.0953; Found: 185.0953.<smiles>COc1cccc(Nc2ncccn2)c1</smiles>

\section{$N$-(3-methoxyphenyl)pyrimidin-2-amine (1m)}

This compound was prepared by reacting 2-chloropyrimidine (1.8 g, $16.2 \mathrm{mmol})$, 4methoxyaniline (2 $\mathrm{g}, 16.2 \mathrm{mmol})$ and conc. $\mathrm{HCl}(1.5 \mathrm{~mL})$. Purification by column chromatography on silica gel (eluent: petroleum ether $\left./ \mathrm{EtOAc}=2 / 1, \mathrm{R}_{\mathrm{f}}=0.33\right)$ gave $1 \mathbf{m}(1.6 \mathrm{~g}$, $49 \%$ ) as a yellow solid. $\mathrm{Mp}=87-88^{\circ} \mathrm{C}$.

${ }^{1} \mathbf{H}$ NMR $\left(\mathrm{CDCl}_{3}, 400 \mathrm{MHz}\right): \delta 8.42(\mathrm{~d}, \mathrm{~J}=4.9 \mathrm{~Hz}, 2 \mathrm{H}), 7.41(\mathrm{t}, \mathrm{J}=2.2 \mathrm{~Hz}, 2 \mathrm{H}), 7.23(\mathrm{t}, \mathrm{J}=$ $7.9 \mathrm{~Hz}, 1 \mathrm{H}), 7.10(\mathrm{ddd}, \mathrm{J}=8.1,2.0,0.9 \mathrm{~Hz}, 1 \mathrm{H}), 6.71(\mathrm{t}, \mathrm{J}=4.8 \mathrm{~Hz}, 1 \mathrm{H}), 6.61(\mathrm{ddd}, \mathrm{J}=8.2$, 2.5, $0.9 \mathrm{~Hz}, 1 \mathrm{H}), 3.83(\mathrm{~s}, 3 \mathrm{H})$.

${ }^{13} \mathrm{C}$ NMR ( $\left.\mathrm{CDCl}_{3}, 100 \mathrm{MHz}\right): \delta 160.4,160.3,158.1,140.8,129.7,112.7,108.0,105.7,55.4$.

IR (neat) cm $\mathrm{cm}^{-1}: 3274$ w, 3181 w, 3109 w, 3065 w, 2361 m, 2339 m, 1739 m, 1605 m, 1574 s, 1533 s, 1492 m, 1444 s, 1427 s, 1405 s, 1263 w, 1249 w, 1199 m, 1157 s, 1079 w, 1047 m, 994 w, 968 w, 852 w, 797 m, 769 m, 690 m.

HRMS (EI) m/z: [M] $]^{+}$Calcd for $\mathrm{C}_{11} \mathrm{H}_{11} \mathrm{~N}_{3} \mathrm{O}$ : 201.0902; Found: 201.0901.<smiles>Cc1cc(C)cc(Nc2ncccn2)c1</smiles>

\section{$N$-(3,5-dimethylphenyl)pyrimidin-2-amine (1n)}

This compound was prepared by reacting 2-chloropyrimidine (2.8 g, $24.7 \mathrm{mmol}), 3,5-$ dimethylaniline (3 g, $24.7 \mathrm{mmol})$ and conc. $\mathrm{HCl}(2.5 \mathrm{~mL})$. Purification by column chromatography on silica gel (eluent: petroleum ether/EtOAc $\left.=3 / 1, \mathrm{R}_{\mathrm{f}}=0.46\right)$ gave $1 \mathrm{n}(1.7 \mathrm{~g}$, $34 \%$ ) as a light brown solid. $\mathrm{Mp}=93-94{ }^{\circ} \mathrm{C}$.

${ }^{1} \mathbf{H}$ NMR $\left(\mathrm{CDCl}_{3}, 400 \mathrm{MHz}\right): \delta 8.40(\mathrm{~d}, \mathrm{~J}=4.8 \mathrm{~Hz}, 2 \mathrm{H}), 7.27$ (brs, 1H), 7.23 (s, 2H), 6.71-6.70 $(\mathrm{m}, 1 \mathrm{H}), 6.68(\mathrm{t}, \mathrm{J}=4.9 \mathrm{~Hz}, 1 \mathrm{H}), 2.32(\mathrm{~s}, 6 \mathrm{H})$. 
${ }^{13} \mathrm{C}$ NMR $\left(\mathrm{CDCl}_{3}, 100 \mathrm{MHz}\right): \delta 160.4,158.1,139.3,138.7,124.8,117.6,112.4,21.6$.

IR (neat) cm $\mathrm{cm}^{-1}: 3272$ w, 3183 w, 3107 w, 3014 w, 2969 w, 2945 w, 2917 w, 2360 s, 2339 m, 1739 m, 1613 m, 1578 s, 1536 s, 1443 s, 1403 m, 1376 m, 1300 w, 1244 w, 1215 w, 1172 w, 1039 w, 991 w, 884 w, 837 m, 797 m, 719 w, 684 m.

HRMS (EI) m/z: [M] $]^{+}$Calcd for $\mathrm{C}_{12} \mathrm{H}_{13} \mathrm{~N}_{3}$ : 199.1109; Found: 199.1113.

All dioxazolone derivatives 2a-2o were synthesized accordingly to previously described procedure. . $^{2,3,4,5,6}$

\subsection{Procedure for the Synthesis of $N$-(o-tolyl)pyrimidin-2-amine[D]-1a:}

To an oven dried screw cap tube equipped with a magnetic stir bar were added $N$-(otolyl)pyrimidin-2-amine $1 \mathrm{a}\left(60 \mathrm{mg}, 0.32 \mathrm{mmol} \text {, [Cp* } \mathrm{RhCl}_{2}\right]_{2}(50 \mathrm{mg}, 0.08 \mathrm{mmol})$, $\mathrm{AgSbF}_{6}(27.7 \mathrm{mg}, 0.08 \mathrm{mmol}), 1,2$ dichloroethane $(\mathrm{DCE})(1 \mathrm{~mL})$ and $\mathrm{D}_{2} \mathrm{O}(0.5 \mathrm{~mL})$ in a glove box. The reaction mixture was heated at $100{ }^{\circ} \mathrm{C}$ for $8 \mathrm{~h}$ on aluminum heating block (Thermo Mighty Stirrer HHE-19G-USIII (KPI)), then allowed to cool at room temperature, the resulting mixture was filtered through a celite pad, and the organic phase was then concentrated in vacuo. The residue was purified by column chromatography on silica gel (eluent: petroleum ether/EtOAc $=5 / 1)$ to afford the desired product, deuterated 1 a $(51 \mathrm{mg}, 84 \%)$. Based on ${ }^{1} \mathrm{H}$ NMR spectra, a $87 \%$ deuterium incorporation was achieved.

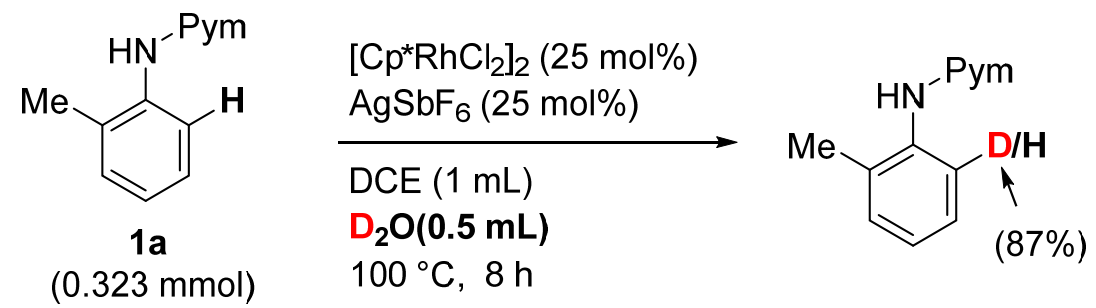




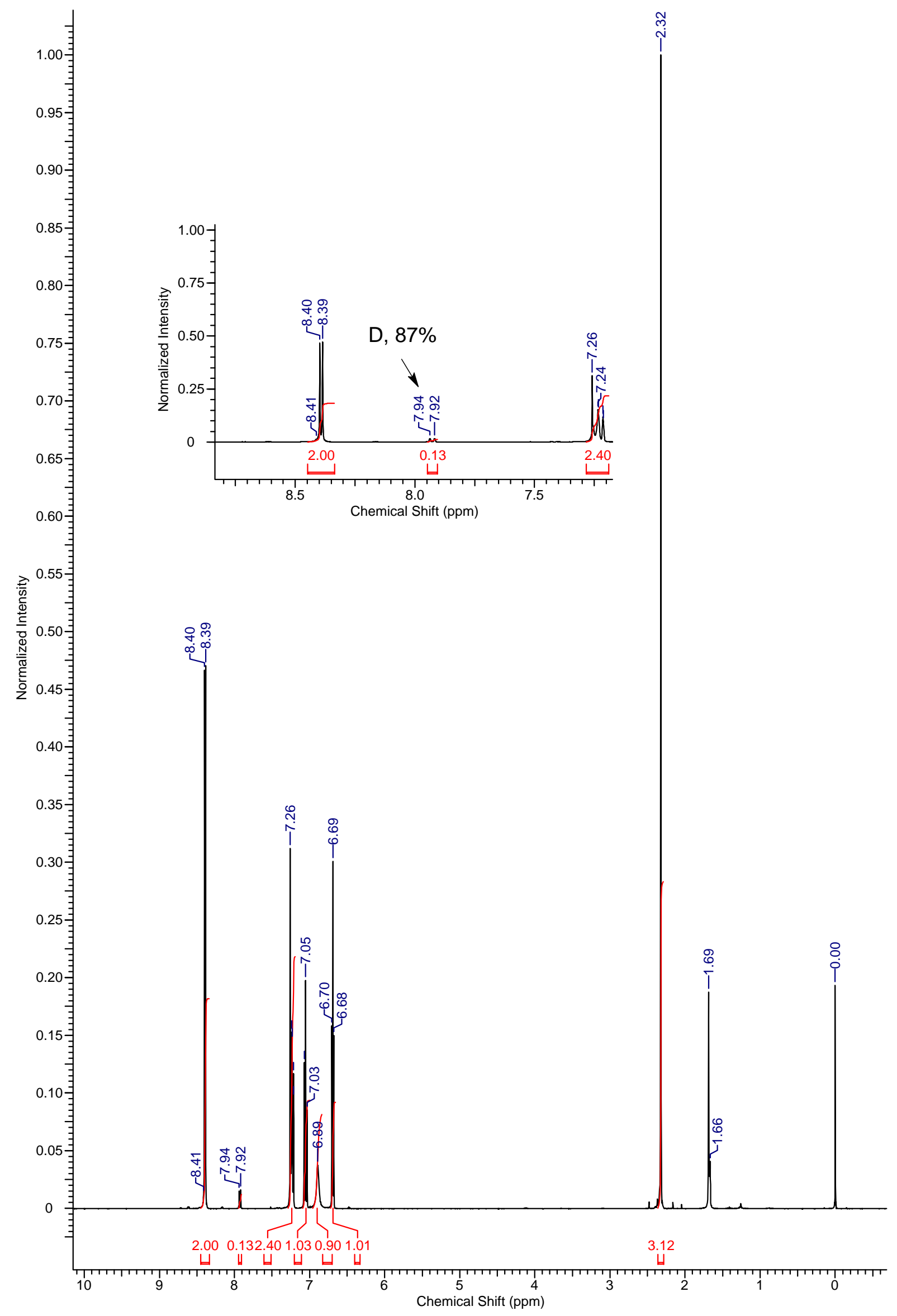




\section{Optimization of the Reactions Conditions}

We started optimizing the reaction conditions for $\mathrm{C}-\mathrm{H}$ bond amidation using $\mathrm{N}-(2-$ tolyl)pyrimidin-2-amine (1a) as a model substrate with 3-phenyl-1,4,2-dioxazol-5-one (2a) (3 equiv) as an amidating reagent (Table S1). When the reaction was carried out in presence of $\left[\mathrm{Cp}^{*} \mathrm{RhCl}_{2}\right]_{2}(5 \mathrm{~mol} \%)$ as the catalyst and $\mathrm{AgSbF}_{6}(10 \mathrm{~mol} \%)$ as an additive in 1,2dichloroethane (DCE, $1 \mathrm{~mL}$ ) as a solvent at $100^{\circ} \mathrm{C}$ for $8 \mathrm{~h}$ on aluminum heating block (Thermo Mighty Stirrer HHE-19G-USIII (KPI)) under a nitrogen atmosphere, N-(3-methyl-2(pyrimidin-2-ylamino)phenyl)benzamide (3aa) was obtained in $25 \%$ isolated yield (entry 1 ). This result encouraged us to further pursue optimization studies with an initial focus on solvents (entries 1-6). Among the solvents examined, hexafluoro-2-propanol (HFIP) proved to be the optimal solvent (entry 6). An examination of the effect of silver salts (entries 6-8) indicated that $\mathrm{AgSbF}_{6}$ was the most efficient salt for the reaction (entry 6). The reaction at $80{ }^{\circ} \mathrm{C}$ gave 3aa only in $10 \%$ (entry 9). Increasing the reaction to $120^{\circ} \mathrm{C}$ did not improve the product yield (entry 10). Next, different molar ratios of 2a were examined (entries 11 and 12). It was found that 2 equivalents of $\mathbf{2 a}$ gave the best result (entry 12). A decrease in catalyst loading from 5 to 2 or $3 \mathrm{~mol} \%$ had no effect on the yield of 3aa (entries 13 and 14), while an increase in the catalyst loading to $10 \mathrm{~mol} \%$ resulted in a decreased yield of $\mathbf{3 a a}$ (entry 15). As a control experiment, the reaction was carried out in the absence of $\left[\mathrm{Cp}^{*} \mathrm{RhCl}_{2}\right]_{2}$ or $\mathrm{AgSbF} 6$ and the desired product 3aa was not formed (entries 16 and 17). Thus, the optimal reaction conditions were determined to be as follows; $1 \mathbf{a}(0.15 \mathrm{mmol}), \mathbf{2 a}(0.3 \mathrm{mmol}),\left[\mathrm{Cp}^{*} \mathrm{RhCl}_{2}\right]_{2}(5$ $\mathrm{mol} \%)$ and $\mathrm{AgSbF}_{6}(10 \mathrm{~mol} \%)$ in $\operatorname{HFIP}(1 \mathrm{~mL})$ at $100{ }^{\circ} \mathrm{C}$ for $8 \mathrm{~h}$ (entry 12$)$.

Table S1. Optimization of Reaction Conditions ${ }^{a}$

\begin{tabular}{|c|c|c|c|c|}
\hline & & $\begin{array}{l}\mathrm{Cp}^{*} \mathrm{RhCl}_{2} \\
\mathrm{AgSbF}_{6}\end{array}$ & $\begin{array}{l}\mathrm{mol} \% \\
\mathrm{~mol} \%\end{array}$ & Pym \\
\hline 1a $0.15 \mathrm{mmol}$ & 2a $0.45 \mathrm{~mm}$ & Pym $=p y$ & & 3aа \\
\hline entry & solvent & temp & time & isolated \\
\hline & & $\left({ }^{\circ} \mathrm{C}\right)$ & (hrs) & yield $(\%)^{b}$ \\
\hline 1 & DCE & 100 & 8 & $25(33)$ \\
\hline 2 & 1,4 dioxane & 100 & 8 & trace \\
\hline 3 & toluene & 100 & 8 & trace \\
\hline
\end{tabular}




$\begin{array}{ccccc}4 & \mathrm{CH}_{3} \mathrm{CN} & 100 & 8 & \text { trace } \\ 5 & \mathrm{CF}_{3} \mathrm{CH}_{2} \mathrm{OH} & 100 & 8 & (30) \\ 6 & \text { HFIP } & 100 & 8 & 80(84) \\ 7^{\mathrm{c}} & \text { HFIP } & 100 & 8 & \mathrm{NR} \\ 8^{\mathrm{d}} & \text { HFIP } & 100 & 8 & 72 \\ 9 & \text { HFIP } & 80 & 8 & (10) \\ 10 & \text { HFIP } & 120 & 4 & 81(84) \\ 11^{\mathrm{e}} & \text { HFIP } & 100 & 8 & (87) \\ 12^{\mathrm{f}} & \text { HFIP } & 100 & 8 & 88(94) \\ 13^{\mathrm{g}} & \text { HFIP } & 100 & 8 & (91) \\ 14^{\mathrm{h}} & \text { HFIP } & 100 & 8 & (93) \\ 15^{\mathrm{i}} & \text { HFIP } & 100 & 8 & (50) \\ 16^{\mathrm{j}} & \text { HFIP } & 100 & 8 & \mathrm{NR} \\ 17^{\mathrm{k}} & \text { HFIP } & 100 & 8 & \mathrm{NR}\end{array}$

${ }^{a}$ Reaction conditions: 1a $(0.15 \mathrm{mmol}), \mathbf{2 a}(0.45 \mathrm{mmol}),\left[\mathrm{Cp}^{*} \mathrm{RhCl}_{2}\right]_{2}(5 \mathrm{~mol} \%), \mathrm{AgSbF}_{6}(10 \mathrm{~mol} \%)$ in solvent $(1 \mathrm{~mL}) .{ }^{b} \mathrm{~A}$ number in parenthesis refers to the NMR yield. ${ }^{c}$ Use of AgOAc (10mol\%). ${ }^{d}$ Use of $\mathrm{AgNF}_{2}(10 \mathrm{~mol} \%)$. ${ }^{e} \mathrm{Use}$ of 1.5 equiv of 2a. ${ }^{f} \mathrm{Use}$ of 2 equiv of 2a. ${ }^{g}\left[\mathrm{Cp}^{*} \mathrm{RhCl}_{2}\right]_{2}(2 \mathrm{~mol} \%) .{ }^{h}\left[\mathrm{Cp}^{*} \mathrm{RhCl}_{2}\right]_{2}$ (3 mol \%). ${ }^{i}\left[\mathrm{Cp}^{*} \mathrm{RhCl}_{2}\right]_{2}(10 \mathrm{~mol} \%) .{ }^{j} \mathrm{No}\left[\mathrm{Cp}^{*} \mathrm{RhCl}_{2}\right]_{2} \cdot{ }^{k} \mathrm{No} \mathrm{AgSbF}$.

\section{General Procedure for Rh-Catalyzed C-H Amidation (Scheme 2):}

General procedure for the $\mathrm{C}-\mathrm{H}$ amidation of $\mathrm{N}$-(o-tolyl)pyrimidin-2-amine (1a) with dioxazolone 2a: To an oven dried screw capped tube equipped with magnetic stir bar in a glove box were added $N$-(o-tolyl)pyrimidin-2-amine (1a) $(27.7 \mathrm{mg}, 0.15 \mathrm{mmol}), 3$-phenyl-1,4,2dioxazol-5-one (2a) (48.9 mg, $0.3 \mathrm{mmol}$ ), [Cp* $\left.\mathrm{RhCl}_{2}\right]_{2}$ (4.64 mg, $\left.0.0075 \mathrm{mmol}\right), \mathrm{AgSbF}_{6}(5.15$ $\mathrm{mg}, 0.015 \mathrm{mmol})$ and hexafluoroisopropanol (HFIP) $(1 \mathrm{~mL})$. The resulting reaction mixture was stirred at $100{ }^{\circ} \mathrm{C}$ for $8 \mathrm{~h}$ on aluminum heating block (Thermo Mighty Stirrer HHE-19GUSIII (KPI)), then allowed to cool at room temperature and the resulting mixture was filtered through a celite pad, and the organic phase was then removed by concentration invacuo. The residue was purified by column chromatography on silica gel (eluent: petroleum ether/EtOAc $=2 / 1)$ to afford the desired product 3aa $(41 \mathrm{mg}, 88 \%)$ as a white solid. 


\section{Procedure for Gram Scale Synthesis of 3aa}

To an oven dried screw capped tube equipped with magnetic stir bar in a glove box were added $\mathrm{N}$-(o-tolyl)pyrimidin-2-amine (1a) $(555.7 \mathrm{mg}, 3 \mathrm{mmol}), 3$-phenyl-1,4,2-dioxazol-5-one (2a) (978.8 mg, $6 \mathrm{mmol}),\left[\mathrm{Cp}^{*} \mathrm{RhCl}_{2}\right]_{2}(92.7 \mathrm{mg}, 0.15 \mathrm{mmol}), \mathrm{AgSbF}_{6}(1023 \mathrm{mg}, 0.3 \mathrm{mmol})$ and hexafluoroisopropanol (HFIP) $(2 \mathrm{~mL})$. The resulting reaction mixture was stirred at $100{ }^{\circ} \mathrm{C}$ for $8 \mathrm{~h}$ on aluminum heating block (Thermo Mighty Stirrer HHE-19G-USIII (KPI)), then allowed to cool at room temperature and the resulting mixture was filtered through a celite pad, and the organic phase was then removed by concentration in vacuo. The residue was purified by column chromatography on silica gel (eluent: petroleum ether/EtOAc=2/1) to afford the desired product $\mathbf{3 a a}(750 \mathrm{mg}, 82 \%)$ as a white solid.

\section{Characterization Data of Amidated Compounds}

\section{Characterization Data of Compounds 3}<smiles>Cc1cccc(NC(=O)c2ccccc2)c1Nc1ncccn1</smiles>

\section{$N$-(3-methyl-2-(pyrimidin-2-ylamino)phenyl)benzamide (3aa):}

$88 \%$ yield. (hexane/EtOAc $=2 / 1, \mathrm{R}_{\mathrm{f}}=0.15$ ). White solid. $\mathrm{Mp}=193-194^{\circ} \mathrm{C}$.

${ }^{1} \mathbf{H}$ NMR $\left(\mathrm{CDCl}_{3}, 400 \mathrm{MHz}\right): \delta 8.9$ (brs, $\left.1 \mathrm{H}\right), 8.40(\mathrm{~d}, \mathrm{~J}=6.1 \mathrm{~Hz}, 2 \mathrm{H}), 8.03(\mathrm{~d}, \mathrm{~J}=9.7 \mathrm{~Hz}, 1 \mathrm{H})$, 7.72-7.70 (m, 2H), 7.50-7.46 (m, 1H), 7.41-7.37 (m, 2H), 7.29 (t, J= 7.8 Hz, 1H), 7.11 (d, J = $7.1 \mathrm{~Hz}, 1 \mathrm{H}), 6.76-6.73(\mathrm{~m}, 2 \mathrm{H}), 2.30$ (s, 3H).

${ }^{13} \mathrm{C}$ NMR $\left(\mathrm{CDCl}_{3}, 100 \mathrm{MHz}\right): \delta 165.6,161.6,158.9,135.1,134.9,131.8,128.8,127.5,127.2$, 127.1, 121.8, 112.8, 18.8.

IR (neat) cm $\mathrm{cm}^{-1}: 3262$ w, 3060 w, 2923 w, 2365 w, 2338 w, 1735 w, 1658 m, 1583 s, 1513 s, 1470 m, 1446 s, 1402 s, 1307 m, 1252 m, 1180 w, 1124 w, 990 w, 847 w, 800 m, 780 m, 707 m.

HRMS (EI) m/z: [M] $]^{+}$Calcd for $\mathrm{C}_{18} \mathrm{H}_{16} \mathrm{~N}_{4} \mathrm{O}$ : 304.1323; Found: 304.1319 . 
<smiles>O=C(Nc1ccccc1Nc1ncccn1)c1ccccc1</smiles>

\section{$N$-(2-(pyrimidin-2-ylamino)phenyl)benzamide (3ba):}

This compound was prepared by following general procedure for $\mathrm{C}-\mathrm{H}$ amidation, using $\mathbf{1 b}$ (25.7 $\mathrm{mg}, 0.15 \mathrm{mmol}$ ) and dioxozolone 2a (48.9 $\mathrm{mg}, 0.3 \mathrm{mmol})$. Purification by column chromatography on silica gel (eluent: petroleum ether/EtOAc: $2 / 1, R_{f}=0.14$ ) yielded 3 ba (27 $\mathrm{mg}, 62 \%$ ) as a White solid. $\mathrm{Mp}=172-173^{\circ} \mathrm{C}$.

${ }^{1} \mathbf{H}$ NMR $\left(\mathrm{CDCl}_{3}, 400 \mathrm{MHz}\right): \delta 9.02$ (brs, 1H), 8.42 (d, J = 5.3 Hz, 2H), 7.95 (d, J = $7.0 \mathrm{~Hz}$, $1 \mathrm{H}), 7.81-7.79(\mathrm{~m}, 2 \mathrm{H}), 7.53-7.40(\mathrm{~m}, 4 \mathrm{H}), 7.31(\mathrm{td}, \mathrm{J}=7.3 \mathrm{~Hz}$ and $1.3 \mathrm{~Hz}, 1 \mathrm{H}), 7.23(\mathrm{td}, \mathrm{J}=$ $7.6 \mathrm{~Hz}$ and $1.2 \mathrm{~Hz}, 1 \mathrm{H}), 7.18(\mathrm{brs}, 1 \mathrm{H}), 6.76(\mathrm{t}, \mathrm{J}=4.8 \mathrm{~Hz}, 1 \mathrm{H})$.

${ }^{13} \mathbf{C ~ N M R}\left(\mathrm{CDCl}_{3}, 100 \mathrm{MHz}\right): \delta 165.9,161.1,158.5,134.8,132.2,131.8,131.4,128.7,127.3$, $126.2,125.8,125.2,125.1,112.7$.

IR (neat) $\mathrm{cm}^{-1}: 3263 \mathrm{w}, 3063 \mathrm{w}, 2928 \mathrm{w}, 2856 \mathrm{w}, 1655 \mathrm{~m}, 1581 \mathrm{~s}, 1515 \mathrm{~s}, 1476 \mathrm{~m}, 1441 \mathrm{~s}$, 1406 m, 1306 m, 1267 w, 1222 w, 1183 w, 1027 w, 991 w, 903 w, 798 m, 754 m, 707 m.

HRMS (EI) m/z: [M] $]^{+}$Calcd for $\mathrm{C}_{17} \mathrm{H}_{14} \mathrm{~N}_{4} \mathrm{O}$ : 290.1168; Found: 290.1166 .<smiles>O=C(Nc1ccccc1-c1nc2ccccc2n1-c1ccccn1)c1ccccc1</smiles>

$\mathrm{N}$-(2-(1-(pyrimidin-2-yl)-1H-benzo[d]imidazol-2-yl)phenyl)benzamide (4ba):

$4 \mathbf{b a}\left(5.9 \mathrm{mg}, 10 \%\right.$ yield). (eluent: petroleum ether/EtOAc $\left.=1 / 1, \mathrm{R}_{\mathrm{f}}=0.11\right)$. Yellow solid. $\mathrm{Mp}=198-199^{\circ} \mathrm{C}$.

${ }^{1} \mathbf{H}$ NMR $\left(\mathrm{CDCl}_{3}, 400 \mathrm{MHz}\right): \delta 12.16(\mathrm{brs}, 1 \mathrm{H}), 8.85(\mathrm{~d}, \mathrm{~J}=8.2 \mathrm{~Hz}, 1 \mathrm{H}), 8.79(\mathrm{~d}, \mathrm{~J}=5.0 \mathrm{~Hz}$, 2H), $8.14(\mathrm{dd}, \mathrm{J}=8.0 \mathrm{~Hz}, 1.7 \mathrm{~Hz}, 2 \mathrm{H}), 7.92-7.88(\mathrm{~m}, 2 \mathrm{H}), 7.57-7.49$ (m, 3H), 7.46-7.38 (m, $3 \mathrm{H}), 7.33(\mathrm{t}, \mathrm{J}=4.8 \mathrm{~Hz}, 1 \mathrm{H}), 6.89(\mathrm{~d}, \mathrm{~J}=4.4 \mathrm{~Hz}, 2 \mathrm{H})$.

${ }^{13} \mathbf{C} \mathbf{N M R}\left(\mathrm{CDCl}_{3}, 100 \mathrm{MHz}\right): \delta 165.6,159.1,157.3,151.2,142.3,138.2,135.2,134.7,131.8$, 130.6, 130.3, 128.7, 127.6, 124.9, 124.4, 122.4, 121.8, 119.8, 119.5, 118.2, 112.5 .

IR (neat) cm $\mathrm{cm}^{-1}: 3347$ w, 3136 w, 3059 w, 2365 w, 2335 w, 1672 w, 1594 s, 1567 s, 1541 s, 1496 s, 1450 m, 1418 s, 1309 m, 1177 w, 1076 w, 926 w, 851 w, 825 w, 795 w, 752 m, 700 m, $669 \mathrm{w}$.

HRMS (EI) m/z: [M] $]^{+}$Calcd for $\mathrm{C}_{24} \mathrm{H}_{18} \mathrm{~N}_{5} \mathrm{O}$ : 392.1511; Found: 392.1509 . 
<smiles>COc1cccc(NC(=O)c2ccccc2)c1Nc1ncccn1</smiles>

\section{$N$-(3-methoxy-2-(pyrimidin-2-ylamino)phenyl)benzamide (3ca)}

This compound was prepared by following general procedure for $\mathrm{C}-\mathrm{H}$ amidation, using $\mathbf{1 c}$ (30.2 $\mathrm{mg}, 0.15 \mathrm{mmol})$ and dioxozolone 2a $(48.9 \mathrm{mg}, 0.3 \mathrm{mmol})$. Purification by column chromatography on silica gel (eluent: petroleum ether/EtOAc: $\left.2 / 1, \mathrm{R}_{\mathrm{f}}=0.13\right)$ yielded 3ca (45.6 $\mathrm{mg}, 95 \%$ ) as a white solid. $\mathrm{Mp}=156-157^{\circ} \mathrm{C}$.

${ }^{1} \mathbf{H}$ NMR $\left(\mathrm{CDCl}_{3}, 400 \mathrm{MHz}\right): \delta 9.88$ (brs, $\left.1 \mathrm{H}\right), 8.42$ (d, J = 4.8 Hz, 2H), 7.79-7.74 (m, 3H), 7.46, (tt, J=7.46 Hz, 2.2 Hz, 1H), 7.40-7.36 (m, 2H), 7.26 (t, J=8.3 Hz, 1H), 7.21 (brs, 1H), 6.78-7.74 (m, 2H), 3.85 (s, 3H).

${ }^{13} \mathbf{C}$ NMR $\left(\mathrm{CDCl}_{3}, 100 \mathrm{MHz}\right): \delta 165.6,161.7,158.5,153.1,135.4,133.9,131.61,128.6,127.2$, 126.2, 120.6, 117.3, 112.8, 107.1, 56.0.

IR (neat) cm $\mathrm{cm}^{-1}: 3386 \mathrm{w}, 3246 \mathrm{w}, 3030 \mathrm{w}, 3005 \mathrm{w}, 2963 \mathrm{w}, 2837 \mathrm{w}, 1709$ w, $1669 \mathrm{~m}, 1599 \mathrm{~m}$, 1582 s, 1509 m, 1472 m, 1446 s, 1435 s, 1404 m, 1359 w, 1304 m, 1268 s, 1251 s, 1181 w, 1126 m, 1073 s, 942 w, 908 w, 800 m, 779 m, 754 w, 706 m.

HRMS (EI) m/z: [M] $]^{+}$Calcd for $\mathrm{C}_{18} \mathrm{H}_{16} \mathrm{~N}_{4} \mathrm{O}_{2}: 320.1273$; Found: 320.1274 .<smiles>O=C(Nc1cccc(Cl)c1Nc1ncccn1)c1ccccc1</smiles>

$N$-(3-chloro-2-(pyrimidin-2-ylamino)phenyl)benzamide (3da):

This compound was prepared by following general procedure for $\mathrm{C}-\mathrm{H}$ amidation, using 1d (30.8 $\mathrm{mg}, 0.15 \mathrm{mmol})$ and dioxozolone 2a $(48.9 \mathrm{mg}, 0.3 \mathrm{mmol})$. Purification by column chromatography on silica gel (eluent: petroleum ether/EtOAc: $2 / 1, \mathrm{R}_{\mathrm{f}}=0.13$ ) yielded 3da (45.6 $\mathrm{mg}, 95 \%$ ) as a white solid. $\mathrm{Mp}=177-178^{\circ} \mathrm{C}$.

${ }^{1} \mathbf{H}$ NMR $\left(\mathrm{CDCl}_{3}, 400 \mathrm{MHz}\right): \delta 9.48$ (brs, $\left.1 \mathrm{H}\right), 8.47$ ( d J=4.8 Hz, 2H), 8.13 (q, J = 8.6 Hz, 4.8 $\mathrm{Hz}, 1 \mathrm{H}), 7.74-7.72(\mathrm{~m}, 2 \mathrm{H}), 7.49$ (tt, J = 7.2 Hz, 2.0 Hz, 1H), 7.42-7.38 (m, 2H), 7.30 (s, 1H), 7.29 (d, J=1.0 Hz, 1H), 6.98 (brs, 1H), 6.84 (t, J = 4.8 Hz, 1H).

${ }^{13} \mathbf{C ~ N M R}\left(\mathrm{CDCl}_{3}, 100 \mathrm{MHz}\right): \delta 165.7,161.2,158.8,135.7,134.8,131.9,130.5,128.8,128.2$, $127.3,127.2,125.7,123.2,113.5$. 
IR (neat) cm $\mathrm{cm}^{-1}: 3267$ w, 3037 w, 2979 w, 2921 w, 2859 w, 2357 w, 2340 w, 1706 w, 1655 m, 1607 w, 1583 s, 1526 w, 1502 s, 1469 w, 1445 s, 1401 m, 1357 w, 1304 m, 1254 m, 1185 w, 1118 w, 1090 w, 1021 w, 989 w, 833 w, 800 m, 777 m, 747 m, 684 w.

HRMS (EI): m/z: [M] $]^{+}$Calcd for $\mathrm{C}_{18} \mathrm{H}_{16} \mathrm{~N}_{4} \mathrm{O}_{2}$ : 324.0778; Found: 324.0780 .<smiles>O=C(Nc1cccc(Br)c1Nc1ncccn1)c1ccccc1</smiles>

\section{$N$-(3-bromo-2-(pyrimidin-2-ylamino)phenyl)benzamide (3ea):}

This compound was prepared by following general procedure for $\mathrm{C}-\mathrm{H}$ amidation, using $1 \mathbf{e}$ (37.5 $\mathrm{mg}, 0.15 \mathrm{mmol}$ ) and dioxozolone 2a (48.9 $\mathrm{mg}, 0.3 \mathrm{mmol})$. Purification by column chromatography on silica gel (eluent: petroleum ether/EtOAc: $2 / 1, \mathrm{R}_{\mathrm{f}}=0.3$ ) yielded 3ea (36 $\mathrm{mg}, 65 \%)$ as a white solid.

$\mathrm{Mp}=161-162^{\circ} \mathrm{C}$.

${ }^{1} \mathbf{H}$ NMR $\left(\mathrm{CDCl}_{3}, 400 \mathrm{MHz}\right): \delta 9.37$ (brs, $\left.1 \mathrm{H}\right), 8.45(\mathrm{~d}, \mathrm{~J}=5.1 \mathrm{~Hz}, 2 \mathrm{H}), 8.20(\mathrm{~d}, \mathrm{~J}=8.3 \mathrm{~Hz}$, 1H), 7.72-7.69 (m, 2H), 7.50-7.45 (m, 2H), 7.40-7.36 (m, 2H), 7.24-7.20 (m, 2H), 6.82 (t, J= $4.9 \mathrm{~Hz}, 1 \mathrm{H})$.

${ }^{13} \mathrm{C}$ NMR ( $\left.\mathrm{CDCl}_{3}, 100 \mathrm{MHz}\right): \delta 165.6,161.28,158.8,136.0,134.9,131.9,129.4,128.8,128.7$, $127.9,127.2,123.7,121.2,113.5$.

IR (neat) cm $\mathrm{cm}^{-1}: 3237 \mathrm{w}, 3060 \mathrm{w}, 2923 \mathrm{w}, 2361 \mathrm{w}, 2339 \mathrm{w}, 1710 \mathrm{w}, 1671 \mathrm{~m}, 1580 \mathrm{~s}, 1519 \mathrm{~m}$, 1490 w, 1443 s, 1402 m, 1361 w, 1302 m, 1181 w, 1100 w, 1027 w, 992 w, 798 m, 776 m, 743 w, $707 \mathrm{~m}$.

HRMS (EI) m/z: [M] $]^{+}$Calcd for $\mathrm{C}_{17} \mathrm{H}_{13} \mathrm{BrN}_{4} \mathrm{O}$ : 368.073; Found: 368.0167 .<smiles>O=C(Nc1cccc(C(F)(F)F)c1Nc1ncccn1)c1ccccc1</smiles>

$N$-(2-(pyrimidin-2-ylamino)-3-(trifluoromethyl)phenyl)benzamide (3fa):

This compound was prepared by following general procedure for $\mathrm{C}-\mathrm{H}$ amidation, using $1 \mathbf{f}(35.9$ $\mathrm{mg}, 0.15 \mathrm{mmol})$ and dioxozolone $2 \mathrm{a}(48.9 \mathrm{mg}, 0.3 \mathrm{mmol})$. Purification by column chromatography on silica gel (eluent: petroleum ether/EtOAc: $2 / 1, R_{f}=0.15$ ) yielded 3fa (36 mg, 39\%) as a light yellow solid.

$\mathrm{Mp}=146-147^{\circ} \mathrm{C}$. 
${ }^{1} \mathbf{H}$ NMR ( $\left.\mathrm{CDCl}_{3}, 400 \mathrm{MHz}\right): \delta 9.08$ (brs, $\left.1 \mathrm{H}\right), 8.48$ (s, 1H), 8.45 (d, J = 5.6 Hz, 2H), 7.68-7.65 (m, 2H), 7.54-7.46 (m, 3H), 7.41-7.36 (m, 2H), 6.97 (brs, 1H), 6.83 (t, J = 4.8 Hz, 1H).

${ }^{13} \mathbf{C}$ NMR $\left(\mathrm{CDCl}_{3}, 100 \mathrm{MHz}\right): \delta 165.7,161.2,158.9,136.8,134.6,132.1,128.8,128.1,127.3$, $127.1,122.5(\mathrm{q}, \mathrm{J}=4.9), 113.7$.

${ }^{19} \mathrm{~F} \mathrm{NMR}\left(\mathrm{CDCl}_{3}, 400 \mathrm{MHz}\right): \delta-61.18$.

IR (neat) cm $\mathrm{cm}^{-1}: 3411 \mathrm{w}, 3250 \mathrm{w}, 3061 \mathrm{w}, 2961 \mathrm{w}, 2917 \mathrm{w}, 2373 \mathrm{w}, 2347 \mathrm{w}, 1958 \mathrm{w}, 1894 \mathrm{w}$, 1663 m, 1580 s, 1529 m, 1492 w, 1478 w, 1444 m, 1403 m, 1326 s, 1258 w, 1166 m, 1126 s, 1100 w, 990 w, 910 w, 851 w, 798 m, 762 w, 707 m, 694 w.

HRMS (EI) m/z: [M] $]^{+}$Calcd for $\mathrm{C}_{18} \mathrm{H}_{13} \mathrm{~F}_{3} \mathrm{~N}_{4} \mathrm{O}$ : 358.1041; Found: 358.1037.<smiles>O=C(Nc1ccc2ccccc2c1Nc1ncccn1)c1ccccc1</smiles>

\section{$N$-(1-(pyrimidin-2-ylamino)naphthalen-2-yl)benzamide (3ga):}

This compound was prepared by following general procedure for $\mathrm{C}-\mathrm{H}$ amidation, using $\mathbf{1 g}$ (33.2 $\mathrm{mg}, 0.15 \mathrm{mmol}$ ) and dioxozolone $\mathbf{2 a}(48.9 \mathrm{mg}, 0.3 \mathrm{mmol})$. Purification by column chromatography on silica gel (eluent: petroleum ether/EtOAc: $2 / 1, \mathrm{R}_{\mathrm{f}}=0.14$ ) yielded 3ga (35 $\mathrm{mg}, 68 \%$ ) as a white solid.

$\mathrm{Mp}=213-215^{\circ} \mathrm{C}$.

${ }^{1} \mathbf{H}$ NMR ( $\left.\mathrm{CDCl}_{3}, 400 \mathrm{MHz}\right): \delta 9.23$ (brs, $\left.1 \mathrm{H}\right), 8.43(\mathrm{~d}, \mathrm{~J}=6.4 \mathrm{~Hz}, 2 \mathrm{H}), 8.32(\mathrm{~d}, \mathrm{~J}=8.4 \mathrm{~Hz}$, 1H), 7.98-7.96 (m, 1H), 7.90-7.88 (m, 2H), 7.80-7.77 (m, 2H), 7.53-7.40 (m, 5H), 7.24 (brs, 1H), 6.77 (t, J=4.7 Hz, 1H).

${ }^{13} \mathbf{C} \mathbf{N M R}\left(\mathrm{CDCl}_{3}, 100 \mathrm{MHz}\right): \delta 165.8,161.8,158.9,134.9,132.4,132.0,131.9,130.5,128.8$, $128.6,127.6,127.2,126.9,125.6,124.4,123.2,122.3,112.6$.

IR (neat) cm $\mathrm{cm}^{-1}: 3231 \mathrm{w}, 3059$ w, 3005 w, 2929 w, 1708 w, 1662 m, 1629 w, 1582 s, 1524 w, 1502 s, 1488 w, 1446 w, 1429 w, 1415 w, 1383 w, 1289 m, 802 m, 748 w, 708 m, 671 w.

HRMS (EI) m/z: [M] $]^{+}$Calcd for $\mathrm{C}_{21} \mathrm{H}_{16} \mathrm{~N}_{4} \mathrm{O}$ : 340.1324; Found: 340.1329.<smiles>Cc1cc(C)c(NC(=O)c2ccccc2)c(Nc2ncccn2)c1</smiles>

$N$-(3,5-dimethyl-2-(pyrimidin-2-ylamino)phenyl)benzamide (3ha) 
This compound was prepared by following general procedure for $\mathrm{C}-\mathrm{H}$ amidation, using $\mathbf{1 h}$ (29.9 $\mathrm{mg}, 0.15 \mathrm{mmol})$ and dioxozolone 2a $(48.9 \mathrm{mg}, 0.3 \mathrm{mmol})$. Purification by column chromatography on silica gel (eluent: petroleum ether/EtOAc: $\left.2 / 1, \mathrm{R}_{\mathrm{f}}=0.13\right)$ yielded 3 ha $(35.8$ $\mathrm{mg}, 75 \%)$ as a white solid.

$\mathrm{Mp}=159-160^{\circ} \mathrm{C}$.

${ }^{1} \mathbf{H}$ NMR ( $\left.\mathrm{CDCl}_{3}, 400 \mathrm{MHz}\right): \delta 8.77$ (brs, $\left.1 \mathrm{H}\right), 8.39$ (d, J = 6.1 Hz, 2H), 7.89 (s, 1H), 7.71-7.69 (m, 2H), 7.48 (tt, J = 7.4 Hz, 1.3 Hz, 1H), 7.39 (tt, J = 7.7 Hz, 2.0 Hz, 2H), 6.93 (m, 1H), 6.72 (t, J = 4.8 Hz, 1H), 6.58 (brs, 1H), 2.37 (s, 3H), 2.25 (s, 3H).

${ }^{13} \mathbf{C}$ NMR $\left(\mathrm{CDCl}_{3}, 100 \mathrm{MHz}\right): \delta 165.5,161.8,158.8,137.4,135.1,135.0,134.7,131.7,128.7$, $127.9,127.1,126.0,121.8,112.5,21.4,18.6$.

IR (neat) cm $\mathrm{cm}^{-1}: 3257 \mathrm{w}, 3058 \mathrm{w}, 3029 \mathrm{w}, 3004 \mathrm{w}, 2920 \mathrm{w}, 2858 \mathrm{w}, 1710 \mathrm{w}, 1657 \mathrm{~m}, 1584 \mathrm{~s}$, 1530 m, 1507 m, 1445 s, 1403 w, 1360 w, 1271 m, 1158 w, 1127 w, 1034 w, 851w, 799 m, $752 \mathrm{w}, 708 \mathrm{~m}, 672 \mathrm{w}$.

HRMS (EI) m/z: [M] $]^{+}$Calcd for $\mathrm{C}_{19} \mathrm{H}_{18} \mathrm{~N}_{4} \mathrm{O}: 318.1481$; Found: 318.1479 .<smiles>COc1cc(C)c(NC(=O)c2ccccc2)c(Nc2ncccn2)c1</smiles>

$N$-(5-methoxy-3-methyl-2-(pyrimidin-2-ylamino)phenyl)benzamide (3ia):

This compound was prepared by following general procedure for $\mathrm{C}-\mathrm{H}$ amidation, using $\mathbf{1 i}$ (33.2 $\mathrm{mg}, 0.15 \mathrm{mmol})$ and dioxozolone $2 \mathrm{a}(48.9 \mathrm{mg}, 0.3 \mathrm{mmol})$. Purification by column chromatography on silica gel (eluent: petroleum ether/EtOAc: $2 / 1, \mathrm{R}_{\mathrm{f}}=0.26$ ) yielded 3ia (42 $\mathrm{mg}, 83 \%$ ) as a light yellow solid.

$\mathrm{Mp}=163-164{ }^{\circ} \mathrm{C}$.

${ }^{1} \mathbf{H}$ NMR $\left(\mathrm{CDCl}_{3}, 400 \mathrm{MHz}\right): \delta 8.76$ (brs, $\left.1 \mathrm{H}\right), 8.39(\mathrm{~d}, \mathrm{~J}=4.2 \mathrm{~Hz}, 2 \mathrm{H}), 7.86(\mathrm{~d}, \mathrm{~J}=3.1 \mathrm{~Hz}$, 1H), 7.71-7.68 (m, 2H), 7.48 (tt, J = 7.2 Hz, 1.3 Hz, 1H), 7.38 (tt, J = 7.4 Hz, 1.4 Hz, 2H), 6.72 $(\mathrm{t}, \mathrm{J}=4.8 \mathrm{~Hz}, 1 \mathrm{H}), 6.65(\mathrm{~d}, \mathrm{~J}=2.8 \mathrm{~Hz}, 1 \mathrm{H}), 6.59$ (brs, 1H), 3.85 (s, 3H), $2.23(\mathrm{~s}, 3 \mathrm{H})$.

${ }^{13} \mathbf{C ~ N M R}\left(\mathrm{CDCl}_{3}, 100 \mathrm{MHz}\right): \delta 165.5,162.1,158.8,158.6,136.8,136.5,135.0,131.8,128.7$, $127.0,120.7,113.0,112.5,104.9,55.5,18.8$.

IR (neat) cm $\mathrm{cm}^{-1}: 3270$ w, 3059 w, 3000 w, 2959 w, 2924 w, 1709 w, 1661 m, 1582 s, 1528 w, 1466 w, 1442 s, 1400 m, 1358 w, 1333 w, 1260 m, 1222 w, 1195 w, 1031 w, 1016 w, 988 w, $954 \mathrm{w}, 850 \mathrm{w}, 799 \mathrm{~m}, 707 \mathrm{~m}, 662 \mathrm{w}$. 
HRMS (EI) m/z: [M] Calcd for $\mathrm{C}_{19} \mathrm{H}_{18} \mathrm{~N}_{4} \mathrm{O}_{2}$ : 334.1430 ; Found: 334.1421.<smiles>Cc1ccc(Nc2ncccn2)c(NC(=O)c2ccccc2)c1</smiles>

$N$-(5-methyl-2-(pyrimidin-2-ylamino)phenyl)benzamide (3ja):

This compound was prepared by following general procedure for $\mathrm{C}-\mathrm{H}$ amidation, using $\mathbf{1} \mathbf{j}$ (27.7 $\mathrm{mg}, 0.15 \mathrm{mmol})$ and dioxozolone $2 \mathrm{a}(48.9 \mathrm{mg}, 0.3 \mathrm{mmol})$. Purification by column chromatography on silica gel (eluent: petroleum ether/EtOAc: $2 / 1, \mathrm{R}_{\mathrm{f}}=0.18$ ) yielded $\mathbf{3 j a}(36$ $\mathrm{mg}, 79 \%$ ) as a light yellow solid.

$\mathrm{Mp}=173-174^{\circ} \mathrm{C}$.

${ }^{1} \mathbf{H}$ NMR $\left(\mathrm{CDCl}_{3}, 400 \mathrm{MHz}\right): \delta 9.02$ (brs, $\left.1 \mathrm{H}\right), 8.39$ (d, J = $\left.4.8 \mathrm{~Hz}, 2 \mathrm{H}\right), 7.82$ (s, 1H), 7.78-7.76 (m, 2H), 7.51-7.47 (m, 1H), 7.42-7.38 (m, 3H), $7.27(\mathrm{~d}, \mathrm{~J}=7.7 \mathrm{~Hz}, 1 \mathrm{H}), 7.01(\mathrm{dd}, \mathrm{J}=8.0 \mathrm{~Hz}$, $1.7 \mathrm{~Hz}, 1 \mathrm{H}), 6.72(\mathrm{t}, \mathrm{J}=5.4 \mathrm{~Hz}, 1 \mathrm{H}), 2.38(\mathrm{~s}, 3 \mathrm{H})$.

${ }^{13} \mathbf{C ~ N M R}\left(\mathrm{CDCl}_{3}, 100 \mathrm{MHz}\right): \delta 165.8,161.4,158.5,136.6,134.9,132.5,131.8,128.7,128.6$, $127.2,126.5,125.4,125.3,112.5,21.2$.

IR (neat) cm $\mathrm{cm}^{-1}: 3272$ w, 3030 w, 2969 w, 2920 w, 2360 s, 2339 m, 1737 w, 1714 w, 1651 w, 1583 s, 1520 s, 1477 w, 1445 s, 1406 m, 1362 w, 1301 w, 1266 w, 1224 w, 1027 w, 991 w, $911 \mathrm{w}, 870 \mathrm{w}, 800 \mathrm{~m}, 706 \mathrm{~m}, 970 \mathrm{w}$.

HRMS (EI) m/z: [M] $]^{+}$Calcd for $\mathrm{C}_{18} \mathrm{H}_{16} \mathrm{~N}_{4} \mathrm{O}$ : 304.1324; Found: 304.1327.<smiles>COc1ccc(Nc2ncccn2)c(NC(=O)c2ccccc2)c1</smiles>

$N$-(5-methoxy-2-(pyrimidin-2-ylamino)phenyl)benzamide (3ka):

This compound was prepared by following general procedure for $\mathrm{C}-\mathrm{H}$ amidation, using $\mathbf{1 k}$ (30.2 $\mathrm{mg}, 0.15 \mathrm{mmol})$ and dioxozolone 2a $(48.9 \mathrm{mg}, 0.3 \mathrm{mmol})$. Purification by column chromatography on silica gel (eluent: petroleum ether/EtOAc: $\left.1 / 1, R_{f}=0.36\right)$ yielded 3ka (36 mg, $75 \%$ ) as a light yellow solid.

$\mathrm{Mp}=170-171^{\circ} \mathrm{C}$. 
${ }^{1} \mathbf{H}$ NMR $\left(\mathrm{CDCl}_{3}, 400 \mathrm{MHz}\right): \delta 9.02$ (brs, $\left.1 \mathrm{H}\right), 8.39$ (d, J = 5.3 Hz, 2H), 7.81 (d, J = $2.9 \mathrm{~Hz}$, 1H), 7.75 (d, J = 7.6 Hz, 2H), 7.49 (tt, J = 7.2 Hz, 1.1 Hz, 1H), 7.40 (t, J = 7.5 Hz, 2H), 7.26$7.23(\mathrm{~m}, 2 \mathrm{H}), 6.74(\mathrm{td}, \mathrm{J}=8.5 \mathrm{~Hz}, 2.8 \mathrm{~Hz}, 2 \mathrm{H}), 3.85$ (s, 3H).

${ }^{13} \mathbf{C} \mathbf{N M R}\left(\mathrm{CDCl}_{3}, 100 \mathrm{MHz}\right): \delta 165.7,161.8,158.7,158.5,134.9,134.8,131.9,128.8,127.2$, $127.1,122.9,112.6,111.9,108.5,55.7$.

IR (neat) cm $\mathrm{cm}^{-1}: 3251 \mathrm{w}, 3062 \mathrm{w}, 3004 \mathrm{w}, 2959 \mathrm{w}, 2835 \mathrm{w}, 2356 \mathrm{w}, 2338 \mathrm{w}, 1709 \mathrm{w}, 1660 \mathrm{~m}$, 1584 s, 1521 s, 1479 w, 1446 s, 1408 w, 1362 w, 1303 w, 1268 m, 1222 w, 1169 w, 1129 w, 1075 w, 1038 m, 967 w, 894 w, 800 m, 753 w, 706 m, 667 w.

HRMS (EI) m/z: [M] Calcd for $\mathrm{C}_{18} \mathrm{H}_{16} \mathrm{~N}_{4} \mathrm{O}_{2}$ : 320.1273 ; Found: 320.1269 .<smiles>Cc1ccc(NC(=O)c2ccccc2)c(Nc2ncccn2)c1</smiles>

$N$-(4-methyl-2-(pyrimidin-2-ylamino)phenyl)benzamide (3la):

This compound was prepared by following general procedure for $\mathrm{C}-\mathrm{H}$ amidation, using 11 (27.7 $\mathrm{mg}, 0.15 \mathrm{mmol}$ ) and dioxozolone $\mathbf{2 a}(48.9 \mathrm{mg}, 0.3 \mathrm{mmol})$. Purification by column chromatography on silica gel (eluent: petroleum ether/EtOAc: $2 / 1, R_{f}=0.14$ ) followed by GPC yielded 3la (35 mg, 77\%) as a white solid.

$\mathrm{Mp}=183-184{ }^{\circ} \mathrm{C}$.

${ }^{1} \mathbf{H}$ NMR ( $\left.\mathrm{CDCl}_{3}, 400 \mathrm{MHz}\right): \delta 9.03$ (brs, 1H), 8.37 (d, J = 4.3 Hz, 2H), 7.85 (brs, 1H), 7.77 $(\mathrm{d}, \mathrm{J}=7.3 \mathrm{~Hz}, 2 \mathrm{H}), 7.73(\mathrm{~d}, \mathrm{~J}=8.3 \mathrm{~Hz}, 1 \mathrm{H}), 7.47$ (tt, J = 8.0 Hz, 0.9 Hz, 1H), 7.38 (t, J = 7.1 Hz, 2H), $7.25(\mathrm{~d}, \mathrm{~J}=1.3 \mathrm{~Hz}, 1 \mathrm{H}), 7.05(\mathrm{~d}, \mathrm{~J}=6.2 \mathrm{~Hz}, 1 \mathrm{H}), 6.70(\mathrm{t}, \mathrm{J}=5.1 \mathrm{~Hz}, 1 \mathrm{H}), 2.32$ (s, $3 \mathrm{H})$.

${ }^{13} \mathbf{C ~ N M R}\left(\mathrm{CDCl}_{3}, 100 \mathrm{MHz}\right): \delta 165.9,161.1,158.5,135.8,134.8,131.7,131.4,129.5,128.6$, 127.2, 126.9, 125.4, 125.2, 112.5, 21.1 .

IR (neat) cm $\mathrm{cm}^{-1}: 3267 \mathrm{w}, 3057 \mathrm{w}, 2920 \mathrm{w}, 2372 \mathrm{w}, 2341 \mathrm{w}, 1709 \mathrm{w}, 1654 \mathrm{~m}, 1581 \mathrm{~s}, 1522 \mathrm{~s}$, 1481 m, 1445 s, 1417 m, 1359 w, 1309 m, 1253 w, 1221 w, 1182 w, 1075 w, 1028 w, 991 w, $904 \mathrm{w}, 870 \mathrm{w}, 800 \mathrm{~m}, 750 \mathrm{w}, 707 \mathrm{~m}$.

HRMS (EI) m/z: [M] $]^{+}$Calcd for $\mathrm{C}_{18} \mathrm{H}_{16} \mathrm{~N}_{4} \mathrm{O}$ : 304.1324; Found: 304.1325. 
<smiles>COc1ccc(NC(=O)c2ccccc2)c(Nc2ncccn2)c1</smiles>

\section{$N$-(4-methoxy-2-(pyrimidin-2-ylamino)phenyl)benzamide (3ma):}

This compound was prepared by following general procedure for $\mathrm{C}-\mathrm{H}$ amidation, using $\mathbf{1 m}$ (30.2 $\mathrm{mg}, 0.15 \mathrm{mmol})$ and dioxozolone 2a $(48.9 \mathrm{mg}, 0.3 \mathrm{mmol})$. Purification by column chromatography on silica gel (eluent: petroleum ether/EtOAc: $2 / 1, \mathrm{R}_{\mathrm{f}}=0.16$ ) followed by GPC yielded 3ma (22 mg, 46\%) as a white solid.

$\mathrm{Mp}=195-196^{\circ} \mathrm{C}$.

${ }^{1} \mathbf{H}$ NMR $\left(\mathrm{CDCl}_{3}, 400 \mathrm{MHz}\right): \delta 8.74$ (brs, $\left.1 \mathrm{H}\right), 8.41$ (d, J = 4.8 Hz, 2H),7.80 (d, J = $7.5 \mathrm{~Hz}$, 2H), 7.64 (d, J = 8.8 Hz, 1H), 7.55 (brs, 1H), 7.50 (tt, J = 7.2 Hz, 1.5 Hz, 1H), 7.43-7.39 (m, 2H), $7.18(\mathrm{~d}, \mathrm{~J}=2.8 \mathrm{~Hz}, 1 \mathrm{H}), 6.79(\mathrm{dd}, \mathrm{J}=6.1,2.4 \mathrm{~Hz}, 1 \mathrm{H}), 6.74$ (t, J = 4.9 Hz, 1H), 3.81 (s, $3 \mathrm{H})$.

${ }^{13} \mathrm{C}$ NMR $\left(\mathrm{CDCl}_{3}, 100 \mathrm{MHz}\right): \delta 166.1,160.8,158.5,157.9,134.7,133.5,131.8,128.7,127.3$, 126.9, 124.1, 112.8, 111.1, 109.9, 55.7.

IR (neat) cm $\mathrm{cm}^{-1}: 3005$ w, 2969 w, 2914 w, 2360 s, 2339 m, 1867 w, 1844 w, 1712 s, 1650 w, 1556 w, 1540 w, 1520 w, 1509 w, 1488 w, 1420 w, 1359 s, 1219 s, 1092 w, 903 w, 765 w, 719 $\mathrm{w}, 669 \mathrm{w}$.

HRMS (EI) m/z: [M] Calcd for $\mathrm{C}_{18} \mathrm{H}_{16} \mathrm{~N}_{4} \mathrm{O}_{2}$ : 320.1273 ; Found: 320.1268 .<smiles>Cc1ccc(C(=O)Nc2cccc(C)c2Nc2ncccn2)cc1</smiles>

\section{4-methyl- $N$-(3-methyl-2-(pyrimidin-2-ylamino)phenyl)benzamide (3ab):}

This compound was prepared by following general procedure for $\mathrm{C}-\mathrm{H}$ amidation, using $\mathbf{1 a}$ (27.7 $\mathrm{mg}, 0.15 \mathrm{mmol}$ ) and dioxozolone $2 \mathbf{b}(53.2 \mathrm{mg}, 0.3 \mathrm{mmol})$. Purification by column chromatography on silica gel (eluent: petroleum ether/EtOAc: $2 / 1, R_{f}=0.21$ ) yielded 3ab (33 $\mathrm{mg}, 69 \%$ ) as a light brown solid.

$\mathrm{Mp}=200-201^{\circ} \mathrm{C}$. 
${ }^{1} \mathbf{H}$ NMR $\left(\mathrm{CDCl}_{3}, 400 \mathrm{MHz}\right): \delta 8.82$ (brs, $\left.1 \mathrm{H}\right), 8.37$ (d, J = 6.1 Hz, 2H), $8.02(\mathrm{~d}, \mathrm{~J}=8.0 \mathrm{~Hz}$, 1H), 7.59 (d, J = 7.4 Hz, 2H), $7.26(\mathrm{t}, \mathrm{J}=7.8 \mathrm{~Hz}, 1 \mathrm{H}), 7.16$ (d, J = 7.9 Hz, 2H), 7.10-7.08 (m, $2 \mathrm{H}), 6.71(\mathrm{t}, \mathrm{J}=4.9 \mathrm{~Hz}, 1 \mathrm{H}), 2.36(\mathrm{~s}, 3 \mathrm{H}), 2.28(\mathrm{~s}, 3 \mathrm{H})$.

${ }^{13} \mathbf{C} \mathbf{N M R}\left(\mathrm{CDCl}_{3}, 100 \mathrm{MHz}\right): \delta 165.6,161.6,158.8,142.2,135.3,135.1,132.2,129.3,128.7$, $127.4,127.1,126.9,121.5,112.6,21.5,18.7$.

IR (neat) cm $\mathrm{cm}^{-1}: 3259$ w, 3029 w, 2969 w, 2922 w, 1737 w, 1715 w, 1651m, 1607 w, 1583 s, 1523 s, 1504 s, 1470 w, 1445 s, 1401 m, 1318 w, 1305 w, 1186 w, 1118 w, 832 w, 799 w, 778 m, $748 \mathrm{~m}, 691 \mathrm{w}, 676 \mathrm{w}$.

HRMS (EI) m/z: [M] ${ }^{+}$Calcd for $\mathrm{C}_{19} \mathrm{H}_{18} \mathrm{~N}_{4} \mathrm{O}: 318.1481$; Found: 318.1484 .<smiles>Cc1cccc(NC(=O)c2ccc(F)cc2)c1Nc1ncccn1</smiles>

\section{4-fluoro- $N$-(3-methyl-2-(pyrimidin-2-ylamino)phenyl)benzamide (3ac):}

This compound was prepared by following general procedure for $\mathrm{C}-\mathrm{H}$ amidation, using $1 \mathbf{a}$ (27.7 $\mathrm{mg}, 0.15 \mathrm{mmol})$ and dioxozolone $2 \mathrm{c}(54.3 \mathrm{mg}, 0.3 \mathrm{mmol})$. Purification by column chromatography on silica gel (eluent: petroleum ether/EtOAc: $\left.2 / 1, \mathrm{R}_{\mathrm{f}}=0.5\right)$ yielded 3ac (43 $\mathrm{mg}, 89 \%$ ) as a white solid.

$\mathrm{Mp}=159-160^{\circ} \mathrm{C}$.

${ }^{1} \mathbf{H}$ NMR $\left(\mathrm{CDCl}_{3}, 400 \mathrm{MHz}\right): \delta 8.89$ (brs, 1H), $8.40(\mathrm{~d}, \mathrm{~J}=4.7 \mathrm{~Hz}, 2 \mathrm{H}), 7.97(\mathrm{~d}, \mathrm{~J}=7.7 \mathrm{~Hz}$, 1H), 7.73-7.70 (m, 2H), $7.28(\mathrm{t}, \mathrm{J}=8.2 \mathrm{~Hz}, 1 \mathrm{H}), 7.11(\mathrm{~d}, \mathrm{~J}=6.5 \mathrm{~Hz}, 1 \mathrm{H}), 7.09-7.03(\mathrm{~m}, 2 \mathrm{H})$, 6.76-6.74 (m, 2H), $2.3(\mathrm{~s}, 3 \mathrm{H})$.

${ }^{13} \mathbf{C ~ N M R}\left(\mathrm{CDCl}_{3}, 100 \mathrm{MHz}\right): \delta 164.8\left(\mathrm{~d}, \mathrm{~J}_{C-F}=250 \mathrm{~Hz}\right), 164.6,161.6,158.8,135.2,134.8$, $131.3\left(\mathrm{~d}, \mathrm{~J}_{C-F}=3.5 \mathrm{~Hz}\right), 129.5\left(\mathrm{~d}, \mathrm{~J}_{C-F}=10 \mathrm{~Hz}\right), 129.0,127.2\left(\mathrm{~d}, \mathrm{~J}_{C-F}=11.4 \mathrm{~Hz}\right), 121.8,115.7$ $\left(\mathrm{d}, \mathrm{J}_{C-F}=22.9 \mathrm{~Hz}\right), 112.6,18.7$.

${ }^{19}$ F NMR $\left(\mathrm{CDCl}_{3}, 400 \mathrm{MHz}\right): \delta-107.7$.

IR (neat) cm $\mathrm{cm}^{-1}: 3276$ w, 3067 w, 2923 w, 2360 w, 1711 w, 1657 m, 1602 w, 1584 s, 1503 s, 1470 w, 1446 s, 1401 m, 1316 w, 1295 w, 12321 m, 1159 m, 1124 w, 990 w, 909 w, 886 w, $850 \mathrm{~m}, 800 \mathrm{w}, 778 \mathrm{~m}, 759 \mathrm{w}, 662 \mathrm{w}$.

HRMS (EI) m/z: [M] $]^{+}$Calcd for $\mathrm{C}_{18} \mathrm{H}_{15} \mathrm{FN}_{4} \mathrm{O}$ : 322.1230; Found: 322.1233 . 
(c)

4-chloro- $N$-(3-methyl-2-(pyrimidin-2-ylamino)phenyl)benzamide (3ad):

This compound was prepared by following general procedure for $\mathrm{C}-\mathrm{H}$ amidation, using $1 \mathrm{a}$ (27.7 $\mathrm{mg}, 0.15 \mathrm{mmol}$ ) and dioxozolone 2d (59.3 $\mathrm{mg}, 0.3 \mathrm{mmol})$. Purification by column chromatography on silica gel (eluent: petroleum ether/EtOAc: $2 / 1, \mathrm{R}_{\mathrm{f}}=0.34$ ) yielded 3ad (35 $\mathrm{mg}, 69 \%$ ) as a white solid.

$\mathrm{Mp}=131-132{ }^{\circ} \mathrm{C}$.

${ }^{1} \mathbf{H}$ NMR $\left(\mathrm{CDCl}_{3}, 400 \mathrm{MHz}\right): \delta 8.93$ (brs, $\left.1 \mathrm{H}\right), 8.38(\mathrm{~d}, \mathrm{~J}=4.8 \mathrm{~Hz}, 2 \mathrm{H}), 7.96(\mathrm{~d}, \mathrm{~J}=7.8 \mathrm{~Hz}$, 1H), $7.62(\mathrm{dt}, \mathrm{J}=8.7 \mathrm{~Hz}, 1.9 \mathrm{~Hz}, 2 \mathrm{H}), 7.34$ (dt, J = 8.7 Hz, $1.9 \mathrm{~Hz}, 2 \mathrm{H}), 7.26$ (t, J = 7.8 Hz, 1H), 7.10 (dq, J = 7.6 Hz, 1.2 Hz, 0.5 Hz, 1H), 6.92 (brs, 1H), 6.74 (t, J = 4.9 Hz, 1H), 2.29 (s, $3 \mathrm{H})$.

${ }^{13} \mathbf{C}$ NMR $\left(\mathrm{CDCl}_{3}, 100 \mathrm{MHz}\right): \delta$ 164.6, 161.5, 158.8, 138.0, 135.1, 134.6, 133.5, 129.0, 128.9, 128.6, 127.4, 127.3, 121.9, 112.7, 18.7 .

IR (neat) cm $\mathrm{cm}^{-1}: 3275 \mathrm{w}, 3032 \mathrm{w}, 2969 \mathrm{w}, 2921 \mathrm{w}, 2856 \mathrm{w}, 2362 \mathrm{w}, 2338 \mathrm{w}, 1736 \mathrm{w}, 1713 \mathrm{w}$, 1657 m, 1584 s, 1509 s, 1487 s, 1470 w, 1446 s, 1402 m, 1314 m, 1254 w, 1224 w, 1179 w, 1092 m, 1014 w, 885 w, 845 w, 799 m, 781 m, 754 w.

HRMS (EI) m/z: [M] $]^{+}$Calcd for $\mathrm{C}_{18} \mathrm{H}_{15} \mathrm{ClN}_{4} \mathrm{O}$ : 338.0934; Found: 338.0935.<smiles>Cc1cccc(NC(=O)c2ccc(Br)cc2)c1Nc1ncccn1</smiles>

\section{4-bromo-N-(3-methyl-2-(pyrimidin-2-ylamino)phenyl)benzamide ( 3ae):}

This compound was prepared by following general procedure for $\mathrm{C}-\mathrm{H}$ amidation, using $1 \mathrm{a}$ (27.7 $\mathrm{mg}, 0.15 \mathrm{mmol})$ and dioxozolone $2 \mathrm{e}(72.6 \mathrm{mg}, 0.3 \mathrm{mmol})$. Purification by column chromatography on silica gel (eluent: petroleum ether/EtOAc: $\left.2 / 1, \mathrm{R}_{\mathrm{f}}=0.45\right)$ yielded $3 \mathrm{ae}(55$ mg, 96\%) as a white solid.

$\mathrm{Mp}=180-181^{\circ} \mathrm{C}$.

${ }^{1} \mathbf{H}$ NMR $\left(\mathrm{CDCl}_{3}, 400 \mathrm{MHz}\right): \delta 8.95$ (brs, $\left.1 \mathrm{H}\right), 8.40(\mathrm{~d}, \mathrm{~J}=4.9 \mathrm{~Hz}, 2 \mathrm{H}), 7.98$ (d, J = 8.0 Hz, 1H), 7.58-7.51 (m, 4H), 7.29 (d, J = 7.6 Hz, 1H), 7.12 (d, J = 7.5 Hz, 1H), 6.76 (t, J = 4.8 Hz, $1 \mathrm{H}), 6.68(\mathrm{brs}, 1 \mathrm{H}), 2.31(\mathrm{~s}, 3 \mathrm{H})$. 
${ }^{13} \mathbf{C} \mathbf{N M R}\left(\mathrm{CDCl}_{3}, 100 \mathrm{MHz}\right): \delta 164.7,161.5,158.6,135.1,134.6,133.9,131.9,129.1,128.7$, $127.3,126.4,121.8,112.7,18.1$.

IR (neat) cm $\mathrm{cm}^{-1}: 3276$ w, 3030 w, 2969 w, 2921 w, 2361 m, 2339 m, 1737 w, 1715 w, 1654 m, $1586 \mathrm{~s}, 1509$ m, 1485 w, 1470 w, 1446 s, 1402 m, 1363 w, 1314 w, 1254 w, 1225 w, 1091 w, 1071 w, 1010 m, 910 w, 884 w, 842 w, 799 w, 780 m, 718 w, 678 w.

HRMS (EI) m/z: [M] $]^{+}$Calcd for $\mathrm{C}_{18} \mathrm{H}_{15} \mathrm{BrN}_{4} \mathrm{O}$ : 338.0429; Found: 338.0423 .<smiles>Cc1cccc(NC(=O)c2ccccc2Cl)c1Nc1ncccn1</smiles>

2-chloro- $N$-(3-methyl-2-(pyrimidin-2-ylamino)phenyl)benzamide (3af):

This compound was prepared by following general procedure for $\mathrm{C}-\mathrm{H}$ amidation, using $\mathbf{1 a}$ (27.7 $\mathrm{mg}, 0.15 \mathrm{mmol})$ and dioxozolone $2 \mathrm{f}(59.3 \mathrm{mg}, 0.3 \mathrm{mmol})$. Purification by column chromatography on silica gel (eluent: petroleum ether/EtOAc: $\left.2 / 1, \mathrm{R}_{\mathrm{f}}=0.24\right)$ yielded 3af $(49.2$ mg, 97\%) as a white solid.

$\mathrm{Mp}=143-144^{\circ} \mathrm{C}$.

${ }^{1} \mathbf{H}$ NMR $\left(\mathrm{CDCl}_{3}, 400 \mathrm{MHz}\right): \delta 8.78(\mathrm{brs}, 1 \mathrm{H}), 8.33$ (d, J $\left.=4.9 \mathrm{~Hz}, 2 \mathrm{H}\right), 8.12(\mathrm{~d}, \mathrm{~J}=8.4 \mathrm{~Hz}$, 1H), $7.70(\mathrm{~d}, \mathrm{~J}=7.2 \mathrm{~Hz}, 1 \mathrm{H}), 7.34-7.28(\mathrm{~m}, 4 \mathrm{H}), 7.13$ (d, J = $7.7 \mathrm{~Hz}, 1 \mathrm{H}), 6.86$ (brs, 1H), 6.67 $(\mathrm{t}, \mathrm{J}=5.1 \mathrm{~Hz}, 1 \mathrm{H}), 2.27(\mathrm{~s}, 3 \mathrm{H})$.

${ }^{13} \mathbf{C ~ N M R}\left(\mathrm{CDCl}_{3}, 100 \mathrm{MHz}\right): \delta 164.5,161.6,158.6,136.0,135.2,135.1,131.6,130.7,130.6$, $130.3,128.5,127.6,127.2,121.0,112.4,18.6$.

IR (neat) cm $\mathrm{cm}^{-1}: 3255$ w, 3057 w, 3032 w, 2922 w, 2855 w, 2361 w, 2333 w, 1708 m, 1666 m, 1582 s, 1525 w, 1502 m, 1469 w, 1441 s, 1400 s, 1360 w, 1310 m, 1273 w, 1222 w, 1089 w, 1052 w, 1035 w, 990 w, 799 w, 778 s, 747 m, 696 w, 663 w.

HRMS (EI) m/z: [M] Calcd for $\mathrm{C}_{18} \mathrm{H}_{15} \mathrm{ClN}_{4} \mathrm{O}$ : 338.0934; Found: 338.0933 .<smiles>Cc1cccc(NC(=O)c2ccccc2F)c1Nc1ncccn1</smiles>

\section{2-fluoro- $N$-(3-methyl-2-(pyrimidin-2-ylamino)phenyl)benzamide (3ag):}

This compound was prepared by following general procedure for $\mathrm{C}-\mathrm{H}$ amidation, using $1 \mathrm{a}$ $(27.7 \mathrm{mg}, 0.15 \mathrm{mmol})$ and dioxozolone $2 \mathrm{~g}(54.3 \mathrm{mg}, 0.3 \mathrm{mmol})$. Purification by column chromatography on silica gel (eluent: petroleum ether/EtOAc: $2 / 1, R_{\mathrm{f}}=0.23$ ) yielded 3ag (47 mg, 97\%) as a white solid. 
$\mathrm{Mp}=177-178^{\circ} \mathrm{C}$.

${ }^{1} \mathbf{H}$ NMR $\left(\mathrm{CDCl}_{3}, 400 \mathrm{MHz}\right): \delta 9.16\left(\mathrm{~d}, \mathrm{~J}_{\mathrm{H}-\mathrm{F}}=14.0 \mathrm{~Hz}, 1 \mathrm{H}\right), 8.36(\mathrm{~d}, \mathrm{~J}=5.4 \mathrm{~Hz}, 2 \mathrm{H}), 8.14$ $(\mathrm{ddd}, \mathrm{J}=10.3 \mathrm{~Hz}, 4.8 \mathrm{~Hz}, 1.9 \mathrm{~Hz}, 2 \mathrm{H}), 7.46-7.42$ (m, 1H), 7.31-7.23 (m, 2H), 7.13 (dq, J = 7.1 $\mathrm{Hz}, 0.8 \mathrm{~Hz}, 1 \mathrm{H}), 8.02(\mathrm{ddd}, \mathrm{J}=12.1 \mathrm{~Hz}, 8.5 \mathrm{~Hz}, 0.8 \mathrm{~Hz}, 1 \mathrm{H}), 6.86$ (brs, 1H), 6.69 (t, J = 4.7 $\mathrm{Hz}, 1 \mathrm{H}), 2.29$ (s, 3H).

${ }^{13} \mathbf{C ~ N M R}\left(\mathrm{CDCl}_{3}, 100 \mathrm{MHz}\right): \delta 161.7,160.3\left(\mathrm{~d}, \mathrm{~J}_{\mathrm{C}-\mathrm{F}}=229.7 \mathrm{~Hz}\right), 158.7,136.6,135.5,133.6$ $\left(\mathrm{d}, \mathrm{J}_{\mathrm{C}-\mathrm{F}}=7.0 \mathrm{~Hz}\right), 128.3,127.6,127.0,124.9\left(\mathrm{~d}, \mathrm{~J}_{\mathrm{C}-\mathrm{F}}=2.6 \mathrm{~Hz}\right), 121.6\left(\mathrm{~d}, \mathrm{~J}_{\mathrm{C}-\mathrm{F}}=11.7 \mathrm{~Hz}\right), 121.1$, $116.2,115.9,112.4,18.6$.

19F NMR: $\delta-113.67$

IR (neat) cm $\mathrm{cm}^{-1}: 3411 \mathrm{w}, 3234 \mathrm{w}, 3040 \mathrm{w}, 2963 \mathrm{w}, 2921 \mathrm{w}, 2361 \mathrm{w}, 2339 \mathrm{w}, 1709 \mathrm{w}, 1671 \mathrm{~m}$, 1607 w, 1583 s, 1534 w, 1503 w, 1469 m, 1442 s, 1401 s, 1360 w, 1317 s, 1283 m, 1257 w, 1221 w, 1130 w, 1094 m, 907 w, 855 w, 801 m, 778 s, 753 s, 668 w.

HRMS (EI) m/z: [M] $]^{+}$Calcd for $\mathrm{C}_{18} \mathrm{H}_{15} \mathrm{FN} 4 \mathrm{O}$ : 322.1230; Found: 322.1226 .<smiles>Cc1cccc(C(=O)Nc2cccc(C)c2Nc2ncccn2)c1</smiles>

\section{3-methyl- $N$-(3-methyl-2-(pyrimidin-2-ylamino)phenyl)benzamide (3ah):}

This compound was prepared by following general procedure for $\mathrm{C}-\mathrm{H}$ amidation, using $\mathbf{1 a}$ $(27.7 \mathrm{mg}, 0.15 \mathrm{mmol})$ and dioxozolone $\mathbf{2 h}(53.0 \mathrm{mg}, 0.3 \mathrm{mmol})$. Purification by column chromatography on silica gel (eluent: petroleum ether/EtOAc: $2 / 1, \mathrm{R}_{\mathrm{f}}=0.32$ ) yielded 3ah (45 $\mathrm{mg}, 94 \%$ ) as a yellow solid.

$\mathrm{Mp}=140-141^{\circ} \mathrm{C}$.

${ }^{1} \mathbf{H}$ NMR $\left(\mathrm{CDCl}_{3}, 400 \mathrm{MHz}\right): \delta 8.84$ (brs, $\left.1 \mathrm{H}\right), 8.38(\mathrm{~d}, \mathrm{~J}=5.1 \mathrm{~Hz}, 2 \mathrm{H}), 8.02(\mathrm{~d}, \mathrm{~J}=8.0 \mathrm{~Hz}$, 1H), 7.49-7.45 (m, 2H), 7.28-7.21 (m, 3H), 7.15 (brs, 1H), 7.10 (dq, J = 7.8 Hz, 0.7 Hz, 1H), $6.71(\mathrm{t}, \mathrm{J}=4.9 \mathrm{~Hz}, 1 \mathrm{H}), 2.31(\mathrm{~s}, 3 \mathrm{H}), 2.28(\mathrm{~s}, 3 \mathrm{H})$,

${ }^{13} \mathrm{C}$ NMR $\left(\mathrm{CDCl}_{3}, 100 \mathrm{MHz}\right): \delta 165.8,161.5,158.8,138.5,135.2,135.0,132.4,128.8,128.5$, $127.9,127.3,127.0,124.1,121.6,112.5,21.4,16.7>$

IR (neat) cm $\mathrm{cm}^{-1}: 3276$ w, 3029 w, 2969 w, 2920 w, 2361 s, 2339 s, 1737 w, 1717 w, 1654 m, 1584 s, 1521 s, 1509 s, 1470 w, 1446 s, 1401 m, 1312 w, 1214 w, 991 w, 892 w, 801 w, 777 w, $717 \mathrm{~m}, 683 \mathrm{~m}$.

HRMS (EI) m/z: [M] $]^{+}$Calcd for $\mathrm{C}_{19} \mathrm{H}_{18} \mathrm{~N}_{4} \mathrm{O}$ : 318.1481 ; Found: 318.1484 . 
(1)

$N$-(3-methyl-2-(pyrimidin-2-ylamino)phenyl)furan-2-carboxamide (3ai):

This compound was prepared by following general procedure for $\mathrm{C}-\mathrm{H}$ amidation, using $\mathbf{1 a}$ (27.7 $\mathrm{mg}, 0.15 \mathrm{mmol}$ ) and dioxozolone $2 \mathbf{i}(46 \mathrm{mg}, 0.3 \mathrm{mmol})$. Purification by column chromatography on silica gel (eluent: petroleum ether/EtOAc: $1 / 1, \mathrm{R}_{\mathrm{f}}=0.18$ ) yielded 3ai (12 $\mathrm{mg}, 27 \%$ ) as a light yellow solid.

$\mathrm{Mp}=171-172{ }^{\circ} \mathrm{C}$.

${ }^{1} \mathbf{H}$ NMR $\left(\mathrm{CDCl}_{3}, 400 \mathrm{MHz}\right): \delta 9.00$ (brs, $\left.1 \mathrm{H}\right), 8.39(\mathrm{~d}, \mathrm{~J}=5.2 \mathrm{~Hz}, 2 \mathrm{H}), 8.05(\mathrm{~d}, \mathrm{~J}=8.0 \mathrm{~Hz}$, 1H), $7.33(\mathrm{dd}, \mathrm{J}=1.9 \mathrm{~Hz}, 1 \mathrm{~Hz}, 1 \mathrm{H}), 7.27$ (t, J = 7.9 Hz, 1H), $7.16(\mathrm{dd}, \mathrm{J}=3.5 \mathrm{~Hz}, 0.6 \mathrm{~Hz}, 1 \mathrm{H})$, $7.12(\mathrm{dd}, \mathrm{J}=7.7 \mathrm{~Hz}, 1 \mathrm{~Hz}, 1 \mathrm{H}), 6.94$ (brs, 1H), 6.72 (t, J = 4.8 Hz, 1H), 6.47 (dd, J = 3.7 Hz, $2.2 \mathrm{~Hz}, 1 \mathrm{H}), 2.29(\mathrm{~s}, 3 \mathrm{H})$.

${ }^{13} \mathbf{C ~ N M R}\left(\mathrm{CDCl}_{3}, 100 \mathrm{MHz}\right): \delta 161.5,158.8,156.3,148.2,144.2,135.6,134.3,128.4,127.5$, 127.0, 121.1, 115.1, 112.6, 112.4, 18.7.

IR (neat) cm $\mathrm{cm}^{-1}: 3387$ w, 3248 w, 3141 w, 2961 w, 2924 w, 2855 w, 2363 w, 2348 w, 1709 w, $1671 \mathrm{~m}, 1583 \mathrm{~s}, 1528 \mathrm{~m}, 1510 \mathrm{~m}, 1470 \mathrm{~m}, 1444 \mathrm{~s}, 1401 \mathrm{~m}, 1313 \mathrm{~m}, 1272 \mathrm{w}, 1166 \mathrm{w}, 1076 \mathrm{w}$, 1010 w, 992 w, 954 w, 800 w, 778 m, 756 m, 659 w.

HRMS (EI) m/z: [M] Calcd for $\mathrm{C}_{16} \mathrm{H}_{14} \mathrm{~N}_{4} \mathrm{O}_{2}$ : 294.1117; Found: 294.1117.<smiles>CC(=O)Nc1cccc(C)c1Nc1ncccn1</smiles>

$N$-(3-methyl-2-(pyrimidin-2-ylamino)phenyl)acetamide (3aj):

This compound was prepared by following general procedure for $\mathrm{C}-\mathrm{H}$ amidation, using $\mathbf{1 a}$ (27.7 $\mathrm{mg}, 0.15 \mathrm{mmol}$ ) and dioxozolone $\mathbf{2 j}$ (30.3 $\mathrm{mg}, 0.3 \mathrm{mmol})$. Purification by column chromatography on silica gel (eluent: petroleum ether/EtOAc: $1 / 5, \mathrm{R}_{\mathrm{f}}=0.64$ ) yielded 3aj $(32$ $\mathrm{mg}, 88 \%$ ) as a yellow solid.

$\mathrm{Mp}=132-133{ }^{\circ} \mathrm{C}$.

${ }^{1} \mathbf{H}$ NMR $\left(\mathrm{CDCl}_{3}, 400 \mathrm{MHz}\right): \delta 8.45$ (brs, $\left.1 \mathrm{H}\right), 8.28$ (dd, J = 5.3 Hz, $\left.1.2 \mathrm{~Hz}, 2 \mathrm{H}\right), 7.78$ (d, J = $7.7 \mathrm{~Hz}, 1 \mathrm{H}), 7.35$ (brs, 1H), 7.15 (t, J = 7.7 Hz, 1H), 7.05 (d, J = 7.7 Hz, 1H), 6.62 (dt, J = 4.9 Hz, $1.5 \mathrm{~Hz}, 1 \mathrm{H}), 2.19$ (s, 3H), 2.04 (s, 3H). 
${ }^{13} \mathbf{C}$ NMR $\left(\mathrm{CDCl}_{3}, 100 \mathrm{MHz}\right): \delta 169.1,161.4,158.5,136.0,134.8,128.4,127.3,126.8,121.4$, $112.2,24.3,18.7$.

IR (neat) cm $\mathrm{cm}^{-1}: 3250 \mathrm{w}, 3044 \mathrm{w}, 2978 \mathrm{w}, 2961 \mathrm{w}, 2918 \mathrm{w}, 2379 \mathrm{w}, 2349$ w, $1668 \mathrm{~m}, 1585 \mathrm{~s}$, 1566 w, 1506 s, 1470 m, 1445 s, 1403 m, 1371 w, 1307 m, 1255 w, 1223 w, 1178 w, 993 w, $844 \mathrm{w}, 796 \mathrm{~m}, 683 \mathrm{w}, 661 \mathrm{w}$.

HRMS (EI) m/z: [M] ${ }^{+}$Calcd for $\mathrm{C}_{13} \mathrm{H}_{14} \mathrm{~N}_{4} \mathrm{O}$ : 242.1168; Found: 242.1166.<smiles>CCCCCC(=O)Nc1cccc(C)c1Nc1ncccn1</smiles>

$\mathrm{N}$-(3-methyl-2-(pyrimidin-2-ylamino)phenyl)hexanamide (3ak):

This compound was prepared by following general procedure for $\mathrm{C}-\mathrm{H}$ amidation, using $\mathbf{1 a}$ (27.7 $\mathrm{mg}, 0.15 \mathrm{mmol})$ and dioxozolone $\mathbf{2 k}(47 \mathrm{mg}, 0.3 \mathrm{mmol})$. Purification by column chromatography on silica gel (eluent: petroleum ether/EtOAc: $2 / 1, \mathrm{R}_{\mathrm{f}}=0.3$ ) yielded 3ak (40 $\mathrm{mg}, 89 \%$ ) as a light yellow solid.

$\mathrm{Mp}=124-125^{\circ} \mathrm{C}$.

${ }^{1} \mathbf{H}$ NMR $\left(\mathrm{CDCl}_{3}, 400 \mathrm{MHz}\right): \delta 8.33(\mathrm{~d}, \mathrm{~J}=4.9 \mathrm{~Hz}, 2 \mathrm{H}), 8.09$ (brs, $\left.1 \mathrm{H}\right), 7.84(\mathrm{~d}, \mathrm{~J}=6.6 \mathrm{~Hz}$, 1H), 7.25 (brs, 1H), 7.19 (t, J = 7.4 Hz, 1H), 7.05 (d, J = 8.3 Hz, 1H), 6.67 (t, J = 4.8 Hz, 1H), 2.26-2.22 (m, 5H), 1.56 (pent, 2H), 1.26-1.21 (m, 4H), 0.085-0.82 (m, 3H).

${ }^{13} \mathbf{C ~ N M R}\left(\mathrm{CDCl}_{3}, 100 \mathrm{MHz}\right): \delta 171.8,161.5,158.6,135.7,134.9,128.4,127.3,126.8,121.2$, $112.3,37.7,31.3,25.4,22.4,18.6,13.9$.

IR (neat) cm $\mathrm{cm}^{-1}: 3255$ w, 3135 w, 3029 w, 2956 w, 2928 w, 2859 w, 2362 w, 2337 w, 1660 m, 1584 s, 1565 m, 1504 s, 1468 m, 1445 s, 1402 m, 1300 w, 1254 w, 1037 w, 990 w, 927 w, 852 w, $798 \mathrm{~m}, 779 \mathrm{~m}, 680 \mathrm{w}, 662 \mathrm{w}$.

HRMS (EI) m/z: [M] $]^{+}$Calcd for $\mathrm{C}_{17} \mathrm{H}_{22} \mathrm{~N}_{4} \mathrm{O}$ : 298.1494; Found: 298.1789.<smiles>CCCC(=O)Nc1cccc(C)c1Nc1ncccn1</smiles>

$N$-(3-methyl-2-(pyrimidin-2-ylamino)phenyl)butyramide (3al):

This compound was prepared by following general procedure for $\mathrm{C}-\mathrm{H}$ amidation, using $\mathbf{1 a}$ (27.7 $\mathrm{mg}, 0.15 \mathrm{mmol})$ and dioxozolone 21 (38.7 $\mathrm{mg}, 0.3 \mathrm{mmol})$. Purification by column 
chromatography on silica gel (eluent: petroleum ether/EtOAc: $2 / 1, \mathrm{R}_{\mathrm{f}}=0.264$ ) yielded 3al (34 $\mathrm{mg}, 84 \%$ ) as a white solid.

$\mathrm{Mp}=129-130^{\circ} \mathrm{C}$.

${ }^{1} \mathbf{H}$ NMR $\left(\mathrm{CDCl}_{3}, 400 \mathrm{MHz}\right): \delta 8.34$ (d, J = 4.9 Hz, 2H), 8.07 (brs, 1H), 7.85 (d, J = $7.6 \mathrm{~Hz}$, 1H), 7.22-7.18 (m, 2H), $7.05(\mathrm{~d}, \mathrm{~J}=8.0 \mathrm{~Hz}, 1 \mathrm{H}), 6.68(\mathrm{t}, \mathrm{J}=4.8 \mathrm{~Hz}, 1 \mathrm{H}), 2.25-2.21(\mathrm{~m}, 5 \mathrm{H})$, 1.61 (sixt, $\mathrm{J}=15.3,7.3 \mathrm{~Hz}, 2 \mathrm{H}), 0.88$ (t, $\mathrm{J}=7.3 \mathrm{~Hz}, 3 \mathrm{H})$.

${ }^{13} \mathbf{C} \mathbf{N M R}\left(\mathrm{CDCl}_{3}, 100 \mathrm{MHz}\right): \delta 171.7,161.5,158.6,135.8,134.9,128.4,127.3,126.8,121.2$, $112.3,39.6,19.1,18.7,13.7$.

IR (neat) cm $\mathrm{cm}^{-1}: 3268$ w, 3136 w, 3026 w, 2959 w, 2870 w, 2361 w, 2340 w, 1738 w, 1660 w, 1583 s, 1565 s, 1504 s, 1468 s, 1445 s, 1402 m, 1276 w, 1255 w, 1215 m, 1090 w, 897 w, 797 w, $780 \mathrm{~m}, 746 \mathrm{w}, 699 \mathrm{~m}$.

HRMS (EI) m/z: [M] $]^{+}$Calcd for $\mathrm{C}_{15} \mathrm{H}_{18} \mathrm{~N}_{4} \mathrm{O}: 270.1481$; Found: 270.1480 .<smiles>Cc1cccc(NC(=O)CCc2ccccc2)c1Nc1ncccn1</smiles>

\section{$N$-(3-methyl-2-(pyrimidin-2-ylamino)phenyl)-3-phenylpropanamide (3am):}

This compound was prepared by following general procedure for $\mathrm{C}-\mathrm{H}$ amidation, using 1a (27.7 $\mathrm{mg}, 0.15 \mathrm{mmol})$ and dioxozolone $2 \mathrm{~m}(57.4 \mathrm{mg}, 0.3 \mathrm{mmol})$. Purification by column chromatography on silica gel (eluent: petroleum ether/EtOAc: $2 / 1, R_{f}=0.32$ ) yielded 3am (39 $\mathrm{mg}, 78 \%$ ) as a white solid.

$\mathrm{Mp}=153-154^{\circ} \mathrm{C}$.

${ }^{1} \mathbf{H}$ NMR $\left(\mathrm{CDCl}_{3}, 400 \mathrm{MHz}\right): \delta 8.31(\mathrm{~d}, \mathrm{~J}=4.3 \mathrm{~Hz}, 2 \mathrm{H}), 8.03$ (brs, $\left.1 \mathrm{H}\right), 7.79(\mathrm{~d}, \mathrm{~J}=8.3 \mathrm{~Hz}$, 1H), 7.26-7.12 (m, 6H), $7.04(\mathrm{~d}, \mathrm{~J}=7.5 \mathrm{~Hz}, 1 \mathrm{H}), 6.91$ (brs, 1H), $6.66(\mathrm{t}, \mathrm{J}=4.7 \mathrm{~Hz}, 1 \mathrm{H}), 2.94$ (t, J = 7.9 Hz, 2H), $2.55(\mathrm{t}, \mathrm{J}=7.9 \mathrm{~Hz}, 2 \mathrm{H}), 2.20(\mathrm{~s}, 3 \mathrm{H})$.

${ }^{13} \mathbf{C}$ NMR $\left(\mathrm{CDCl}_{3}, 100 \mathrm{MHz}\right): \delta 170.7,161.5,158.6,140.7$ 135.7, 134.7, 128.6, 128.6, 128.4, $128.4,127.3,126.9,126.3,112.4,39.4,31.6,18.7$.

IR (neat) cm $\mathrm{cm}^{-1}: 3270$ w, 3140 w, 3060 w, 3026 w, 2959 w, 2923 w, 2858 w, 1709 w, 1658 w, 1583 s, 1565 w, 1536 w, 1501 s, 1468 w, 1444 s, 1401 s, 1359 m, 1308 w, 1254 w, 1220 m, 1077 w, 1031 w, 990 w, 907 w, 847 w, 796 m, 782 w, 750 m, 699 s, 675 w.

HRMS (EI) m/z: [M] $]^{+}$Calcd for $\mathrm{C}_{20} \mathrm{H}_{20} \mathrm{~N}_{4} \mathrm{O}$ : 332.1637; Found: 332.1642. 
<smiles>Cc1cccc(NC(=O)C2CCCCC2)c1Nc1ncccn1</smiles>

$N$-(3-methyl-2-(pyrimidin-2-ylamino)phenyl)cyclohexanecarboxamide (3an):

This compound was prepared by following general procedure for $\mathrm{C}-\mathrm{H}$ amidation, using $1 \mathrm{a}$ (27.7 $\mathrm{mg}, 0.15 \mathrm{mmol})$ and dioxozolone $2 \mathrm{n}(50.8 \mathrm{mg}, 0.3 \mathrm{mmol})$. Purification by column chromatography on silica gel (eluent: petroleum ether/EtOAc: $2 / 1, R_{f}=0.2124$ ) yielded 3an (39 $\mathrm{mg}, 69 \%)$ as a white solid.

$\mathrm{Mp}=175-176^{\circ} \mathrm{C}$.

${ }^{1} \mathbf{H}$ NMR $\left(\mathrm{CDCl}_{3}, 400 \mathrm{MHz}\right): \delta 8.36(\mathrm{~d}, \mathrm{~J}=4.8 \mathrm{~Hz}, 2 \mathrm{H}), 7.94(\mathrm{brs}, 1 \mathrm{H}), 7.86(\mathrm{~d}, \mathrm{~J}=8.3 \mathrm{~Hz}$, 1H), 7.21 (t, J = 7.8 Hz, 1H), 7.05 (d, J = 7.8 Hz, 1H), 7.01 (brs, 1H), 6.71 (t, J = 4.4 Hz, 1H), $2.24(\mathrm{~s}, 3 \mathrm{H}), 2.14(\mathrm{tt}, \mathrm{J}=11.6,3.7 \mathrm{~Hz}, 1 \mathrm{H}), 1.82(\mathrm{~d}, \mathrm{~J}=13.4 \mathrm{~Hz}, 2 \mathrm{H}), 1.75-1.72(\mathrm{~m}, 2 \mathrm{H}), 1.64-$ $1.61(\mathrm{~m}, 1 \mathrm{H}), 1.39$ (dq, J = 14.9, 3.4 Hz, 2H), 1.28-1.09 (m, 3H).

${ }^{13} \mathbf{C ~ N M R}\left(\mathrm{CDCl}_{3}, 100 \mathrm{MHz}\right): \delta 174.5,161.6,158.7,135.7,135.0,128.4,127.4,126.7,121.3$, $112.4,46.3,29.6,25.8,25.7,18.7$.

IR (neat) cm $\mathrm{cm}^{-1}: 3272$ w, 3026 w, 2967 w, 2929 m, 2855 w, 2360 s, 2339 s, 1738 s, 1669 w, 1584 s, 1566 w, 1508 s, 1470 w, 1446 s, 1403 w, 1373 w, 1216 s, 798 w, 782 w, 697 w, 670 $\mathrm{w}, 655 \mathrm{w}$.

HRMS (EI) m/z: [M] $]^{+}$Calcd for $\mathrm{C}_{18} \mathrm{H}_{22} \mathrm{~N}_{4} \mathrm{O}: 310.1794$; Found: 310.1792 .

\section{General Procedure for Rh-Catalyzed C-H Amidation Followed by Cyclization (Scheme 3 and 4):}

To an oven dried screw cap tube equipped with magnetic stir bar in a glove box, were added $\mathrm{N}$-phenylpyrimidin-2-amine (1b) (25.7 mg, $0.15 \mathrm{mmol})$, 3-phenyl-1,4,2-dioxazol-5-one2a (73.4 mg, $0.45 \mathrm{mmol}$ ), [Cp* RhCl $]_{2}$ (4.64 mg, $\left.0.0075 \mathrm{mmol}\right), \mathrm{AgSbF}_{6}(15.5 \mathrm{mg}, 0.045 \mathrm{mmol})$ and trifluoroethanol $\left(\mathrm{CF}_{3} \mathrm{CH}_{2} \mathrm{OH}\right)(1 \mathrm{~mL})$. The resulting reaction mixture was stirred at $120^{\circ} \mathrm{C}$ for $18 \mathrm{~h}$ on aluminum heating block (Thermo Mighty Stirrer HHE-19G-USIII (KPI)), then allowed to cool to room temperature, the resulting mixture was filtered through a celite pad, and the organic phase was then concentrated in vacuo. The residue was purified by column chromatography on silica gel (eluent: petroleum ether/EtOAc $=3 / 1, \mathrm{R}_{\mathrm{f}}=0.18$ ) to afford the desired product (5ba) (29 $\mathrm{mg}, 71 \%$ ) as an oily liquid. 


\section{Characterization Data for Cyclized Product 5}<smiles>c1ccc(-c2nc3ccccc3n2-c2ncccn2)cc1</smiles>

\section{2-phenyl-1-(pyrimidin-2-yl)-1H-benzo[d]imidazole(5ba):}

(29 $\mathrm{mg}, 71 \%$ ) as an oily liquid.

${ }^{1} \mathbf{H}$ NMR $\left(\mathrm{CDCl}_{3}, 400 \mathrm{MHz}\right): \delta 8.75(\mathrm{~d}, \mathrm{~J}=5.2 \mathrm{~Hz}, 2 \mathrm{H}), 8.01(\mathrm{ddd}, \mathrm{J}=7.4 \mathrm{~Hz}, 1.6 \mathrm{~Hz}, 0.7 \mathrm{~Hz}$, 1H), 7.90 (ddd, J = 6.3 Hz, 2.6 Hz, 0.6 Hz, 1H), 7.55-7.53 (m, 2H), 7.41-7.35 (m, 5H), 7.26 (t, $\mathrm{J}=4.3 \mathrm{~Hz}, 1 \mathrm{H})$.

${ }^{13} \mathbf{C}$ NMR $\left(\mathrm{CDCl}_{3}, 100 \mathrm{MHz}\right): \delta 158.8,157.2,153.2,143.3,135.1,131.5,129.4,129.2,128.3$, $124.3,123.9,120.2,119.2,112.7$.

IR (neat) cm $\mathrm{cm}^{-1}: 3056 \mathrm{w}, 2924 \mathrm{w}, 2361 \mathrm{w}, 2340 \mathrm{w}, 1737 \mathrm{w}, 1716 \mathrm{w}, 1649 \mathrm{w}, 1609 \mathrm{w}, 1566 \mathrm{~s}$, 1526 w, 1477 w, 1453 s, 1420 s, 1374 w, 1333 m, 1261 w, 1213 w, 1156 w, 1011 w, 986 w, 912 w, 841 w, 809 w, 766 m, 745 m, 697 m.

HRMS (EI) m/z: [M] $]^{+}$Calcd for $\mathrm{C}_{17} \mathrm{H}_{12} \mathrm{~N}_{4}$ : 272.1062; Found: 272.1057.<smiles>Cc1ccc(-c2nc3ccccc3n2-c2ncccn2)cc1</smiles>

\section{1-(pyrimidin-2-yl)-2-(p-tolyl)-1H-benzo[d] imidazole (5bb):}

This compound was prepared by following general procedure for $\mathrm{C}-\mathrm{H}$ amidation/cyclization, using $1 \mathbf{b}(25.7 \mathrm{mg}, 0.15 \mathrm{mmol})$ and dioxozolone $\mathbf{2 b}(79.7 \mathrm{mg}, 0.45 \mathrm{mmol})$. Purification by column chromatography on silica gel (eluent: petroleum ether/EtOAc: $2 / 1, R_{f}=0.26$ ) yielded 5bb (25.05 $\mathrm{mg}, 58 \%)$ as a light yellow solid.

$\mathrm{Mp}=124-125^{\circ} \mathrm{C}$.

${ }^{1} \mathbf{H}$ NMR $\left(\mathrm{CDCl}_{3}, 400 \mathrm{MHz}\right): \delta 8.76(\mathrm{~d}, \mathrm{~J}=4.4 \mathrm{~Hz}, 2 \mathrm{H}), 7.95-7.93(\mathrm{~m}, 1 \mathrm{H}), 7.88-7.86(\mathrm{~m}, 1 \mathrm{H})$, $7.43(\mathrm{dt}, \mathrm{J}=8.3 \mathrm{~Hz}, 1.8 \mathrm{~Hz}, 2 \mathrm{H}), 7.39-7.31(\mathrm{~m}, 2 \mathrm{H}), 7.25$ (t, J = 5.0 Hz, 1H), 7.16 (dd, J = 7.9 $\mathrm{Hz}, 1 \mathrm{~Hz}, 2 \mathrm{H}), 2.38$ (s, 3H).

${ }^{13} \mathbf{C}$ NMR $\left(\mathrm{CDCl}_{3}, 100 \mathrm{MHz}\right): \delta 158.8,157.3,153.3,143.3,139.6,135.2,129.1,129.0,128.5$, $124.1,123.9,120.0,119.2,112.5,21.5$. 
IR (neat) cm $\mathrm{cm}^{-1}: 3029 \mathrm{w}, 2923 \mathrm{w}, 2856 \mathrm{w}, 1737 \mathrm{w}, 1717 \mathrm{w}, 1636 \mathrm{w}, 1616 \mathrm{w}, 1565 \mathrm{~m}, 1541 \mathrm{w}$, 1521 w, 1507 w, 1486 w, 1452 m, 1419 s, 1374 m, 1329 m, 1261 w, 1214 w, 1011 w, 845 w, $822 \mathrm{~m}, 765 \mathrm{w}, 746 \mathrm{~m}, 653 \mathrm{w}$.

HRMS (EI) m/z: [M] $]^{+}$Calcd for $\mathrm{C}_{18} \mathrm{H}_{14} \mathrm{~N}_{4}$ : 286.1218; Found: 286.1213 .<smiles>Fc1ccc(-c2nc3ccccc3n2-c2ncccn2)cc1</smiles>

\section{2-(4-fluorophenyl)-1-(pyrimidin-2-yl)-1H-benzo[d]imidazole (5bc)}

This compound was prepared by following general procedure for $\mathrm{C}-\mathrm{H}$ amidation/cyclization, using $\mathbf{1 b}(25.7 \mathrm{mg}, 0.15 \mathrm{mmol})$ and dioxozolone $\mathbf{2 c}(81.5 \mathrm{mg}, 0.45 \mathrm{mmol})$. Purification by column chromatography on silica gel (eluent: petroleum ether/EtOAc: $2 / 1, \mathrm{R}_{\mathrm{f}}=0.34$ ) yielded 5bc (33 mg, 75\%) as a light yellow crystalline solid.

$\mathrm{Mp}=198-199^{\circ} \mathrm{C}$.

${ }^{1} \mathbf{H}$ NMR $\left(\mathrm{CDCl}_{3}, 400 \mathrm{MHz}\right): \delta 8.77(\mathrm{~d}, \mathrm{~J}=5.1 \mathrm{~Hz}, 2 \mathrm{H}), 8.02-7.98(\mathrm{~m}, 1 \mathrm{H}), 7.89-7.85(\mathrm{~m}, 1 \mathrm{H})$, 7.56-7.51 (m, 2H), 7.41-7.34 (m, 2H), 7.28 (t, J = 4.8 Hz, 1H), $7.07(\mathrm{tt}, \mathrm{J}=8.7 \mathrm{~Hz}, 2.8 \mathrm{~Hz}, 2 \mathrm{H})$. ${ }^{13} \mathbf{C}$ NMR $\left(\mathrm{CDCl}_{3}, 100 \mathrm{MHz}\right): \delta 163.5\left(\mathrm{~d}, \mathrm{~J}_{\mathrm{C}-\mathrm{F}}=256.8 \mathrm{~Hz}\right), 158.8,157.2,152.3,143.2,135.1$, $131.2,131.3\left(\mathrm{~d}, \mathrm{~J}_{\mathrm{C}-\mathrm{F}}=8.8 \mathrm{~Hz}\right), 127.9\left(\mathrm{~d}, \mathrm{~J}_{\mathrm{C}-\mathrm{F}}=3.1 \mathrm{~Hz}\right), 124.4,124.1,120.2,119.3,115.6,115.3$, 112.9.

${ }^{19}$ F NMR: $\delta-111.09$

IR (neat) cm $\mathrm{cm}^{-1}: 3058$ w, 2925 w, 2794 w, 2365 w, 2339 w, 2169 w, 1896 w, 1711 w, 1596 w, 1567 s, 1535 w, 1486 m, 1452 s, 1422 s, 1374 m, 1332 m, 1280 w, 1261 w, 1216 s, 1164 m, 1074 w, 1009 w, 989 w, 969 w, 847 s, 809 s, 764 w, 744 s, 678 w, 654 w.

HRMS (EI) m/z: [M] Calcd for $\mathrm{C}_{17} \mathrm{H}_{11} \mathrm{FN}_{4}$ : 290.0968; Found: 290.0966.<smiles>Clc1ccc(-c2nc3ccccc3n2-c2ncccn2)cc1</smiles>

2-(4-chlorophenyl)-1-(pyrimidin-2-yl)-1H-benzo[d]imidazole (5bd)

This compound was prepared by following general procedure for $\mathrm{C}-\mathrm{H}$ amidation/cyclization, using $1 \mathbf{b}(25.7 \mathrm{mg}, 0.15 \mathrm{mmol})$ and dioxozolone $2 \mathbf{d}(89 \mathrm{mg}, 0.45 \mathrm{mmol})$. Purification by 
column chromatography on silica gel (eluent: petroleum ether/EtOAc: $3 / 1, \mathrm{R}_{\mathrm{f}}=0.33$ ) yielded 5bd (36 mg, 78\%) as a yellow solid.

$\mathrm{Mp}=148-149{ }^{\circ} \mathrm{C}$.

${ }^{1} \mathbf{H}$ NMR $\left(\mathrm{CDCl}_{3}, 400 \mathrm{MHz}\right): \delta 8.77(\mathrm{~d}, \mathrm{~J}=5.2 \mathrm{~Hz}, 2 \mathrm{H}), 8.04-7.99(\mathrm{~m}, 1 \mathrm{H}), 7.89-7.85(\mathrm{~m}, 1 \mathrm{H})$, $7.49(\mathrm{dt}, \mathrm{J}=8.2 \mathrm{~Hz}, 3.0 \mathrm{~Hz}, 2 \mathrm{H}), 7.41-7.34(\mathrm{~m}, 4 \mathrm{H}), 7.29$ (t, J = 5.2 Hz, 1H),

${ }^{13} \mathbf{C ~ N M R}\left(\mathrm{CDCl}_{3}, 100 \mathrm{MHz}\right): \delta 158.8,157.1,152.1,143.2,135.6,135.1,130.6,130.3,128.6$, $124.5,124.1,120.2,119.3,112.9$.

IR (neat) cm $\mathrm{cm}^{-1}: 3059$ w, 2978 w, 2925 w, 2854 w, 2358 w, 2327 w, 1900 w, 1813 w, 1710 w, 1566 s, 1521 w, 1477 m, 1450 s, 1420 s, 1403 w, 1374 m, 1334 m, 1261 w, 1199 w, 1183 w, 1133 w, 1090 m, 1009 m, 987 w, 933 w, 916 w, 838 m, 811 m, 727 w, 744 s, 727 m, 683 w.

HRMS (EI) m/z: [M] $]^{+}$Calcd for $\mathrm{C}_{17} \mathrm{H}_{11} \mathrm{ClN}_{4}$ : 306.0672; Found: 306.0671.<smiles>Brc1ccc(-c2nc3ccccc3n2-c2ncccn2)cc1</smiles>

\section{2-(4-bromophenyl)-1-(pyrimidin-2-yl)-1H-benzo[d]imidazole (5be)}

This compound was prepared by following general procedure for $\mathrm{C}-\mathrm{H}$ amidation/cyclization, using $\mathbf{1 b}$ (25.7 mg, $0.15 \mathrm{mmol})$ and dioxozolone $2 \mathrm{e}$ (109 $\mathrm{mg}, 0.45 \mathrm{mmol})$. Purification by column chromatography on silica gel (eluent: petroleum ether/EtOAc: $3 / 1, \mathrm{R}_{\mathrm{f}}=0.34$ ) yielded $\mathbf{5 b b}(30.02 \mathrm{mg}, 58 \%)$ as a yellow solid.

$\mathrm{Mp}=142-143^{\circ} \mathrm{C}$.

${ }^{1} \mathbf{H}$ NMR $\left(\mathrm{CDCl}_{3}, 400 \mathrm{MHz}\right): \delta 8.77(\mathrm{~d}, \mathrm{~J}=4.1 \mathrm{~Hz}, 2 \mathrm{H}), 8.05-7.99(\mathrm{~m}, 1 \mathrm{H}), 7.90-7.84(\mathrm{~m}, 1 \mathrm{H})$, $7.51(\mathrm{dt}, \mathrm{J}=6.3 \mathrm{~Hz}, 2.2 \mathrm{~Hz}, 2 \mathrm{H}), 7.42(\mathrm{dt}, \mathrm{J}=6.7 \mathrm{~Hz}, 2.1 \mathrm{~Hz}, 2 \mathrm{H}), 7.40-7.35$ (m, 2H), 7.29 (t, $\mathrm{J}=4.9 \mathrm{~Hz}, 1 \mathrm{H})$.

${ }^{13} \mathbf{C}$ NMR $\left(\mathrm{CDCl}_{3}, 100 \mathrm{MHz}\right): \delta 158.8,157.1,152.2,143.2,135.1,131.5,130.8,130.7,124.6$, 124.1, 123.9, 120.2, 119.3, 112.9 .

IR (neat) cm $\mathrm{cm}^{-1}: 3054$ w, 2985 w, 2923 w, 2363 w, 1710 w, 1672 w, 1564 s, 1522 w, 1476 w, 1451 m, 1419 s, 1399 w, 1372 w, 1329 m, 1262 w, 1215 w, 1182 w, 1151 w, 1133 w, 1070 w, 1008 w, 986 w, 934 w, 830 m, 764 w, 746 s, 726 w, 710 w.

HRMS (EI) m/z: [M] ${ }^{+}$Calcd for $\mathrm{C}_{17} \mathrm{H}_{11} \mathrm{BrN}_{4}$ : 350.0167; Found: 350.0169 . 


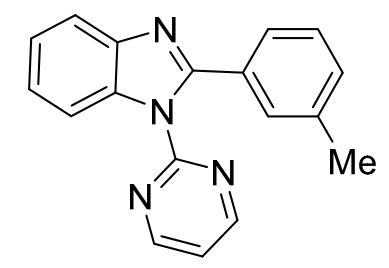

\section{1-(pyrimidin-2-yl)-2-(m-tolyl)-1H-benzo[d]imidazole (5bh):}

This compound was prepared by following general procedure for $\mathrm{C}-\mathrm{H}$ amidation/cyclization, using $\mathbf{1 b}$ (25.7 mg, $0.15 \mathrm{mmol})$ and dioxozolone $\mathbf{2 h}(79.7 \mathrm{mg}, 0.45 \mathrm{mmol})$. Purification by column chromatography on silica gel (eluent: petroleum ether/EtOAc: $3 / 1, R_{f}=0.28$ ) yielded 5bh (29 mg, 67\%) as a light yellow crystalline solid.

$\mathrm{Mp}=103-104{ }^{\circ} \mathrm{C}$.

${ }^{1} \mathbf{H}$ NMR $\left(\mathrm{CDCl}_{3}, 400 \mathrm{MHz}\right): \delta 8.76(\mathrm{~d}, \mathrm{~J}=5.2 \mathrm{~Hz}, 2 \mathrm{H}), 7.98-7.94(\mathrm{~m}, 1 \mathrm{H}), 7.90-7.85(\mathrm{~m}, 1 \mathrm{H})$, $7.56(\mathrm{~s}, 1 \mathrm{H}), 7.40-7.33(\mathrm{~m}, 2 \mathrm{H}), 7.27(\mathrm{td}, \mathrm{J}=5.0 \mathrm{~Hz}, 1 \mathrm{~Hz}, 1 \mathrm{H}), 7.23-7.17$ (m, 2H), 7.14 (t, dt, $\mathrm{J}=5.0 \mathrm{~Hz}, 1.6 \mathrm{~Hz}, 1 \mathrm{H}), 2.37(\mathrm{~s}, 3 \mathrm{H})$.

${ }^{13} \mathbf{C} \mathbf{N M R}\left(\mathrm{CDCl}_{3}, 100 \mathrm{MHz}\right): \delta 158.8,157.3,153.4,143.3,138.3,135.2,131.3,130.3,129.8$, $128.0,126.3,124.2,123.9,120.1,119.2,112.6,21.5$.

IR (neat) cm $\mathrm{cm}^{-1}: 3044$ w, 2978 w, 2919 w, 2855 w, 2791 w, 2736 w, 2363 w, 2339 w, 1943 w, 1895 w, 1608 w, 1565 s, 1522 w, 1481 w, 1451 s, 1417 s, 1373 w, 1326 s, 1261 w, 11999 w, 1078 w, 1027 w, 1008 w, 925 w, 911 w, 881 w, 853 w, 816 w, 788 m, 764 w, 744 s, 696 m.

HRMS (EI) m/z: [M] $]^{+}$Calcd for $\mathrm{C}_{18} \mathrm{H}_{14} \mathrm{~N}_{4}$ : 286.1218; Found: 286.1211.<smiles>c1cnc(-n2c(-c3ccco3)nc3ccccc32)nc1</smiles>

\section{2-(furan-2-yl)-1-(pyrimidin-2-yl)-1H-benzo[d]imidazole (5bi):}

This compound was prepared by following general procedure for $\mathrm{C}-\mathrm{H}$ amidation/cyclization, using $\mathbf{1 b}(25.7 \mathrm{mg}, 0.15 \mathrm{mmol})$ and dioxozolone $2 \mathbf{i}(68.9 \mathrm{mg}, 0.45 \mathrm{mmol})$. Purification by column chromatography on silica gel (eluent: petroleum ether/EtOAc: $2 / 1, \mathrm{R}_{\mathrm{f}}=0.16$ ) yielded 5bi(13 mg, 33\%) as a yellow solid. $\mathrm{Mp}=126-127^{\circ} \mathrm{C}$.

${ }^{1} \mathbf{H}$ NMR $\left(\mathrm{CDCl}_{3}, 400 \mathrm{MHz}\right): \delta 8.85(\mathrm{~d}, \mathrm{~J}=5.0 \mathrm{~Hz}, 2 \mathrm{H}), 7.91(\mathrm{ddd}, \mathrm{J}=6.9 \mathrm{~Hz}, 2.5 \mathrm{~Hz}, 0.7 \mathrm{~Hz}$, 1H), 7.87 (ddd, J = 7.5 Hz, 1.7 Hz, 0.8 Hz, 1H), 7.40 (dd, J = 1.8 Hz, 0.9 Hz, 1H), 7.38-7.32 (m, 3H), 6.54 (dd, J = 3.4 Hz, $1.8 \mathrm{~Hz}, 1 \mathrm{H})$.

${ }^{13} \mathbf{C} \mathbf{N M R}\left(\mathrm{CDCl}_{3}, 100 \mathrm{MHz}\right): \delta 158.8,156.8,145.3,143.9,143.9,143.2,134.7,124.5,124.1$, $120.2,119.6,112.8,112.5,111.8$. 
IR (neat) cm $\mathrm{cm}^{-1}: 1618 \mathrm{w}, 1566 \mathrm{~m}, 1513 \mathrm{w}, 1473 \mathrm{w}, 1452 \mathrm{~m}, 1418 \mathrm{~s}, 1374 \mathrm{w}, 1328 \mathrm{~m}, 1263 \mathrm{w}$, 1173 w, 1025 w, 1010 w, 899 w, 826 w, 791 w, 746 s, 714 w, 666 w.

HRMS (EI) m/z: [M] $]^{+}$Calcd for $\mathrm{C}_{15} \mathrm{H}_{10} \mathrm{~N}_{4} \mathrm{O}$ : 262.0855; Found: 262.0853.<smiles>c1cnc(-n2c(C3CCCCC3)nc3ccccc32)nc1</smiles>

\section{2-cyclohexyl-1-(pyrimidin-2-yl)-1H-benzo[d]imidazole (5bn):}

This compound was prepared by following general procedure for $\mathrm{C}-\mathrm{H}$ diamidation/cyclization, using $1 \mathbf{b}(25.7 \mathrm{mg}, 0.15 \mathrm{mmol})$ and dioxozolone 2n (76.2 $\mathrm{mg}, 0.45 \mathrm{mmol})$. Purification by column chromatography on silica gel (eluent: petroleum ether/EtOAc: $10 / 1, \mathrm{R}_{\mathrm{f}}=0.562$ ) yielded 5bn (31 mg, 74\%) as a white yellow solid

$$
\mathrm{Mp}=110-111^{\circ} \mathrm{C} \text {. }
$$

${ }^{1} \mathbf{H}$ NMR $\left(\mathrm{CDCl}_{3}, 400 \mathrm{MHz}\right): \delta 8.87(\mathrm{~d}, \mathrm{~J}=4.7 \mathrm{~Hz}, 2 \mathrm{H}), 8.10-8.06(\mathrm{~m}, 1 \mathrm{H}), 7.81-7.77(\mathrm{~m}, 1 \mathrm{H})$, 7.33-7.26 (m,3H), 3.71 (tt, J = 11.7 Hz, 3.3 Hz, 1H), 2.09 (d, J = 13.3 Hz, 2H), 1.87-1.75 (m, $5 \mathrm{H}), 1.43-1.33(\mathrm{~m}, 3 \mathrm{H})$.

${ }^{13} \mathbf{C ~ N M R}\left(\mathrm{CDCl}_{3}, 100 \mathrm{MHz}\right): \delta 160.1,158.6,157.1,142.8,134.0,123.4,123.4,119.4,118.6$, 113.6, 37.9, 32.1, 26.5, 26.11.

IR (neat) cm $\mathrm{cm}^{-1}: 2360 \mathrm{~s}, 2339$ s, 1738 s, 1568 w, 1521 w, 1509 w, 1455 w, 1422 m, 1366 m, $1228 \mathrm{~m}, 1215 \mathrm{~m}, 670 \mathrm{w}$.

HRMS (EI) m/z: [M] $]^{+}$Calcd for $\mathrm{C}_{17} \mathrm{H}_{18} \mathrm{~N}_{4}$ : 278.1531; Found: 278.1536.

\section{General Procedure for the Rh-Catalyzed C-H Diamidation followed by Cyclization (Scheme 4):}

To an oven dried screw cap tube equipped with magnetic stir bar in a glove box, were added $\mathrm{N}$-phenylpyrimidin-2-amine (1b) $(25.7 \mathrm{mg}, 0.15 \mathrm{mmol}), 3$-methyl-1,4,2-dioxazol-5-one (2j) (45.4 mg, $0.45 \mathrm{mmol}$ ), [Cp* $\left.\mathrm{RhCl}_{2}\right]_{2}$ (4.64 mg, $\left.0.0075 \mathrm{mmol}\right), \mathrm{AgSbF}_{6}(15.5 \mathrm{mg}, 0.045 \mathrm{mmol})$ and trifluoroethanol $\left(\mathrm{CF}_{3} \mathrm{CH}_{2} \mathrm{OH}\right)(1 \mathrm{~mL})$. The resulting reaction mixture was stirred at $120^{\circ} \mathrm{C}$ for $18 \mathrm{~h}$ on aluminum heating block (Thermo Mighty Stirrer HHE-19G-USIII (KPI)), then allowed to cool to room temperature, the resulting mixture was filtered through a celite pad, and the organic phase was then concentrated in vacuo. The residue was purified by column 
chromatography on silica gel (eluent: petroleum ether/EtOAc $=1 / 5, R_{f}=0.27$ ) to afford the desired product (6bj) (35 mg, 87\%) as a light yellow solid.

\subsection{Characterization Data for Cyclized Product 6}<smiles>CC(=O)Nc1cccc2nc(C)n(-c3ncccn3)c12</smiles>

$N$-(2-methyl-1-(pyrimidin-2-yl)-1H-benzo[d]imidazol-7-yl)acetamide (6bj):

(35 mg, 87\%) as a yellow solid. $\mathrm{Mp}=201-202^{\circ} \mathrm{C}$.

${ }^{1} \mathbf{H}$ NMR $\left(\mathrm{CDCl}_{3}, 400 \mathrm{MHz}\right): \delta 9.45$ (brs, $\left.1 \mathrm{H}\right), 8.84$ (d, J = 4.9 Hz, 2H), 7.76 (d, J = $7.8 \mathrm{~Hz}$, 1H), $7.47(\mathrm{~d}, \mathrm{~J}=8.0 \mathrm{~Hz}, 1 \mathrm{H}), 7.34(\mathrm{t}, \mathrm{J}=4.8 \mathrm{~Hz}, 1 \mathrm{H}), 7.25$ (t, J = 8.0 Hz,1H), $2.70(\mathrm{~s}, 3 \mathrm{H}), 1.90$ $(\mathrm{s}, 3 \mathrm{H})$.

${ }^{13} \mathrm{C}$ NMR $\left(\mathrm{CDCl}_{3}, 100 \mathrm{MHz}\right): \delta 167.79,158.6,156.8,153.3,144.2,125.1,123.9,123.7,119.3$, $119.2,116.3,24.5,17.19$.

IR (neat) cm $\mathrm{cm}^{-1}: 3249$ w, 3040 w, 2935 w, 2858 w, 1737 w, 1719 w, 1669 m, 1621 w, 1596 w, 1568 s, 1544 w, 1427 s, 1416 s, 1383 w, 1367 w, 1320 w, 1302 w, 1272 w, 1226 w, 1014 w, $961 \mathrm{w}, 913 \mathrm{w}, 825$ w, 789 m, $731 \mathrm{~s}, 701 \mathrm{w}, 667 \mathrm{w}$.

HRMS (EI) m/z: [M] $]^{+}$Calcd for $\mathrm{C}_{14} \mathrm{H}_{13} \mathrm{~N}_{5} \mathrm{O}: 267.1120$; Found: 267.1123.

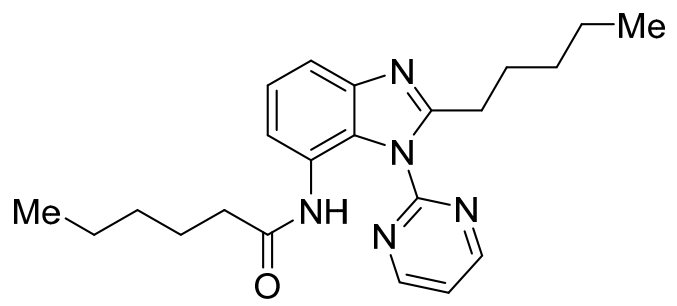

$N$-(2-pentyl-1-(pyrimidin-2-yl)-1H-benzo[d]imidazol-7-yl)hexanamide (6bk):

This compound was prepared by following general procedure for $\mathrm{C}-\mathrm{H}$ diamidation/cyclization, using $\mathbf{1 b}$ (25.7 mg, $0.15 \mathrm{mmol})$ and dioxozolone 2k (70.7 mg, $0.45 \mathrm{mmol})$. Purification by column chromatography on silica gel (eluent: petroleum ether/EtOAc: $2 / 1, R_{f}=0.22$ ) yielded 6bk (47 $\mathrm{mg}, 82 \%)$ as an oily liquid.

${ }^{1} \mathbf{H}$ NMR (CDCl $\left.3,400 \mathrm{MHz}\right): \delta 9.09$ (brs, $\left.1 \mathrm{H}\right), 8.91$ (d, J = $\left.6.0 \mathrm{~Hz}, 2 \mathrm{H}\right), 7.79$ (d, J = 7.6 Hz, 1H), $7.58(\mathrm{~d}, \mathrm{~J}=7.1 \mathrm{~Hz}, 1 \mathrm{H}), 7.41(\mathrm{t}, \mathrm{J}=6.0 \mathrm{~Hz}, 1 \mathrm{H}), 7.30(\mathrm{t}, \mathrm{J}=8.0 \mathrm{~Hz}, 1 \mathrm{H}), 3.07(\mathrm{t}, \mathrm{J}=7.8$ $\mathrm{Hz}, 1 \mathrm{H}$ ), 2.13-2.07 (m, 3H), 1.73 (quentet, $\mathrm{J}=14.5 \mathrm{~Hz}, 6.9 \mathrm{~Hz}, 2 \mathrm{H}$ ), 1.53 (quentet, $\mathrm{J}=14.5 \mathrm{~Hz}$, $7.5 \mathrm{~Hz}, 2 \mathrm{H}), 1.36-1.11(\mathrm{~m}, 8 \mathrm{H}), 0.87-0.84(\mathrm{~m}, 6 \mathrm{H})$. 
${ }^{13} \mathbf{C}$ NMR ( $\left.\mathrm{CDCl}_{3}, 100 \mathrm{MHz}\right): \delta 170.9,158.6,156.9,156.8,144.2,125.5,123.5,119.3,119.2$, $116.4,37.7,31.6,31.4,29.8,27.5,25.3,22.4,22.3,14.0$.

IR (neat) cm $\mathrm{cm}^{-1}: 3270 \mathrm{w}, 3036 \mathrm{w}, 2954 \mathrm{w}, 2929 \mathrm{w}, 2859$ w, $2360 \mathrm{w}, 2339 \mathrm{w}, 1738 \mathrm{w}, 1716 \mathrm{w}$, 1671 w, 1617 w, 1595 w, 1565 m, 1521 w, 1459 w, 1415 s, 1376 w, 1292 w, 1227 w, 1216 w, 1065 w, 967 w, 813 w, 791 m, 736 s, 670 w.

HRMS (EI) m/z: [M] $]^{+}$Calcd for $\mathrm{C}_{22} \mathrm{H}_{29} \mathrm{~N}_{5} \mathrm{O}: 379.2372$; Found: 379.2369.<smiles>CCCc1nc2cccc(NC(=O)CCO)c2n1-c1ncccn1</smiles>

$N$-(2-propyl-1-(pyrimidin-2-yl)-1H-benzo[d] imidazol-7-yl)butyramide (6bl):

This compound was prepared by following general procedure for $\mathrm{C}-\mathrm{H}$ diamidation/cyclization, using $1 \mathbf{b}$ (25.7 mg, $0.15 \mathrm{mmol})$ and dioxozolone 21 (38.7 $\mathrm{mg}, 0.45 \mathrm{mmol})$. Purification by column chromatography on silica gel (eluent: petroleum ether/EtOAc: $1 / 2, \mathrm{R}_{\mathrm{f}}=0.182$ ) yielded 6bl (36 mg, 74\%) as a light yellow solid

$\mathrm{Mp}=85-86{ }^{\circ} \mathrm{C}$.

${ }^{1} \mathbf{H}$ NMR $\left(\mathrm{CDCl}_{3}, 400 \mathrm{MHz}\right): \delta 9.12$ (brs, $\left.1 \mathrm{H}\right), 8.92(\mathrm{~d}, \mathrm{~J}=4.9 \mathrm{~Hz}, 2 \mathrm{H}), 7.81$ (d, J = 8.4 Hz, 1H), $7.58(\mathrm{~d}, \mathrm{~J}=7.7 \mathrm{~Hz}, 1 \mathrm{H}), 7.42(\mathrm{t}, \mathrm{J}=4.2 \mathrm{~Hz}, 1 \mathrm{H}), 7.31(\mathrm{t}, \mathrm{J}=8.0 \mathrm{~Hz}, 1 \mathrm{H}), 3.06$ (t, J = 8.2 $\mathrm{Hz}, 2 \mathrm{H}$ ), 2.08 (t, J = 7.5 Hz,2H), 1.77 (sixtet, J = 14.6 Hz, 7.1 Hz, 2H), 1.58 (sixtet, J = 15.3 $\mathrm{Hz}, 7.8 \mathrm{~Hz}, 2 \mathrm{H}), 0.96$ (t, J = 7.4 Hz, 3H), $0.86(\mathrm{t}, \mathrm{J}=7.4 \mathrm{~Hz}, 3 \mathrm{H})$.

${ }^{13} \mathrm{C}$ NMR $\left(\mathrm{CDCl}_{3}, 100 \mathrm{MHz}\right): \delta 170.8,158.6,156.9,156.6,144.3,125.5,123.6,119.3,119.2$, $116.4,39.6,31.8,21.2,19.0,14.0,13.7$.

IR (neat) cm $\mathrm{cm}^{-1}: 3262$ w, 3029 w, 2962 w, 2932 w, 2872 w, 2361 w, 2341 w, 1737 w, 1717 w, 1682 w, 1596 w, 1567 s, 1522 w, 1508 w, 1455 w, 1427 s, 1416 s, 1374 w, 1291 w, 1227 w, 1216 m, 1091 w, 1067 w, 812 w, 739 m, 677 w, 668 w, 656 w.

HRMS (EI) m/z: [M] $]^{+}$Calcd for $\mathrm{C}_{14} \mathrm{H}_{13} \mathrm{~N}_{5} \mathrm{O}$ : 323.1746; Found: 323.1751 .<smiles>O=C(CCc1ccccc1)Nc1cccc2nc(CCc3ccccc3)n(-c3ncccn3)c12</smiles>

$N$-(2-phenethyl-1-(pyrimidin-2-yl)-1H-benzo[d]imidazol-7-yl)-3-phenylpropanamide (6bm): 
This compound was prepared by following general procedure for C-H diamidation/cyclization, using $1 \mathbf{b}(25.7 \mathrm{mg}, 0.15 \mathrm{mmol})$ and dioxozolone $\mathbf{2 m}(86 \mathrm{mg}, 0.45 \mathrm{mmol})$. Purification by column chromatography on silica gel (eluent: petroleum ether/EtOAc: $1 / 2, \mathrm{R}_{\mathrm{f}}=0.2124$ ) yielded 6bm (65 mg, 97\%) as a white yellow solid

$\mathrm{Mp}=133-134^{\circ} \mathrm{C}$.

${ }^{1} \mathbf{H}$ NMR $\left(\mathrm{CDCl}_{3}, 400 \mathrm{MHz}\right): \delta 9.24$ (brs, $\left.1 \mathrm{H}\right), 8.81(\mathrm{~d}, \mathrm{~J}=4.7 \mathrm{~Hz}, 2 \mathrm{H}), 7.85(\mathrm{~d}, \mathrm{~J}=8.0 \mathrm{~Hz}$, 1H), $7.62(\mathrm{~d}, \mathrm{~J}=7.8 \mathrm{~Hz}, 1 \mathrm{H}), 7.37-7.13(\mathrm{~m}, 12 \mathrm{H}), 3.41-3.37(\mathrm{~m}, 2 \mathrm{H}), 3.19-3.15(\mathrm{~m}, 2 \mathrm{H}), 2.93$ $(\mathrm{t}, \mathrm{J}=7.0 \mathrm{~Hz}, 2 \mathrm{H}), 2.44-2.40(\mathrm{~m}, 2 \mathrm{H})$.

${ }^{13} \mathbf{C}$ NMR ( $\left.\mathrm{CDCl}_{3}, 100 \mathrm{MHz}\right): \delta 169.8,158.4,156.6,155.8,144.2,141.1,140.9,128.6,128.5$, $128.4,128.3,126.3,126.2,125.3,123.8,123.5,119.3,119.2,116.6,39.6,34.2,32.1,31.4$.

IR (neat) cm $\mathrm{cm}^{-1}: 3265 \mathrm{w}, 3059$ w, $3026 \mathrm{w}, 2928 \mathrm{w}, 2858 \mathrm{w}, 2360 \mathrm{w}, 2339 \mathrm{w}, 1738 \mathrm{w}, 1711 \mathrm{w}$, 1671 w, 1596 w, 1566 s, 1538 w, 1495 w, 1451 w, 1417 s, 1375 w, 1269 w, 1218 w, 1159 w, 1029 w, 969 w, 909 w, 816 w, 791 w, 740 m, 700 s, 657 w.

HRMS (EI) m/z: [M] ${ }^{+}$Calcd for $\mathrm{C}_{28} \mathrm{H}_{25} \mathrm{~N}_{5} \mathrm{O}$ : 447.2059; Found: 447.2064.

\subsection{Characterization Data for Cyclized Product 7}<smiles>O=C1CCCN(c2ncccn2)c2ccccc2N1</smiles>

\section{6-(pyrimidin-2-yl)-3,4,5,6-tetrahydrobenzo $[b][1,4]$ diazocin-2(1H)-one (7):}

This compound was prepared by following general procedure for $\mathrm{C}-\mathrm{H}$ diamidation/cyclization, using $1 \mathbf{b}$ (25.7 mg, $0.15 \mathrm{mmol})$ and dioxozolone $2 \mathbf{0}(73.6 \mathrm{mg}, 0.45 \mathrm{mmol})$. Purification by column chromatography on silica gel (eluent: petroleum ether/EtOAc: $1 / 1, \mathrm{R}_{\mathrm{f}}=0.234$ ) yielded $7(30 \mathrm{mg}, 78 \%)$ as an oily liquid.

${ }^{1} \mathbf{H}$ NMR $\left(\mathrm{CDCl}_{3}, 400 \mathrm{MHz}\right): \delta 8.37(\mathrm{~d}, \mathrm{~J}=4.9 \mathrm{~Hz}, 2 \mathrm{H}), 8.01(\mathrm{dd}, \mathrm{J}=8.0,1.4 \mathrm{~Hz}, 1 \mathrm{H}), 7.78$ (brs, 1H), $7.31(\mathrm{td}, \mathrm{J}=7.9,1.4 \mathrm{~Hz}, 1 \mathrm{H}), 7.21(\mathrm{dd}, \mathrm{J}=8.0,1.5 \mathrm{~Hz}, 1 \mathrm{H}), 7.14-7.10(\mathrm{~m}, 1 \mathrm{H}), 6.67$ $(\mathrm{t}, \mathrm{J}=4.7 \mathrm{~Hz}, 1 \mathrm{H}), 3.84(\mathrm{t}, \mathrm{J}=6.9 \mathrm{~Hz}, 2 \mathrm{H}), 2.62(\mathrm{~d}, \mathrm{~J}=7.9 \mathrm{~Hz}, 2 \mathrm{H}), 2.21-2.16(\mathrm{~m}, 3 \mathrm{H})$.

${ }^{13} \mathrm{C}$ NMR $\left(\mathrm{CDCl}_{3}, 100 \mathrm{MHz}\right): \delta 174.3,160.6,158.2,134.8,130.6,127.6,124.8,124.4,124.3$, 112.6, 51.0, 31.7, 19.3. 
IR (neat) cm ${ }^{-1}: 3285$ w, 3032 w, 2669 w, 2890 w, 2360 m, 2339 m, 1737 w, 1675 s, 1577 s, 1564 w, 1517 s, 1478 w, 1460 w, 1438 s, 1400 s, 1302 s, 1221 s, 1139 w, 988 w, 931 w, 904 w, $842 \mathrm{~m}, 755 \mathrm{~s}, 672 \mathrm{~m}$.

HRMS (EI) m/z: [M] $]^{+}$Calcd for $\mathrm{C}_{14} \mathrm{H}_{14} \mathrm{~N}_{4} \mathrm{O}$ : 254.1168; Found: 254.1172 .

\section{Chemoselective Deprotection}

9.1 General Procedure for the Deprotection of an Amide Group of $N$-(3-methyl-2(pyrimidin-2-ylamino)phenyl)benzamide (3aa) to Form 6-methyl- $N^{1}$-(pyrimidin-2yl)benzene-1,2-diamine (8) (Scheme 8).

To an oven dried screw cap tube equipped with magnetic stir bar were added $N$-(3-methyl-2(pyrimidin-2-ylamino)phenyl)benzamide (3aa) (60 mg, $0.20 \mathrm{mmol}), \mathrm{KOH}$ (442 mg, 7.89 $\mathrm{mmol})$, and ethanol $(2 \mathrm{~mL})$. The resulting reaction mixture was stirred at $80{ }^{\circ} \mathrm{C}$ for $24 \mathrm{~h}$ on aluminum heating block (Thermo Mighty Stirrer HHE-19G-USIII (KPI)) under air. After cooling to room temperature, the reaction mixture was diluted with $\mathrm{H}_{2} \mathrm{O}(10 \mathrm{~mL})$ and extracted with EtOAc $(4 \times 20 \mathrm{~mL})$. The combined organic layers were dried over $\mathrm{MgSO}_{4}$ and concentrated in vacuo. The crude product was purified by flash column chromatography on silica gel (eluent: petroleum ether/EtOAc $=1: 2)$ to give $8(32 \mathrm{mg}, 83 \%)$ as a pale yellow solid.<smiles>Cc1cccc(N)c1Nc1ncccn1</smiles>

\section{6-methyl-N1-(pyrimidin-2-yl)benzene-1,2-diamine (8)}

$83 \%$ yield. $R_{f}=0.35$ (hexane/EtOAc $=1 / 2$ ). Light yellow solid. $\mathrm{Mp}=193-194^{\circ} \mathrm{C}$.

${ }^{1} \mathbf{H}$ NMR $\left(\mathrm{CDCl}_{3}, 400 \mathrm{MHz}\right): \delta 8.35(\mathrm{~d}, \mathrm{~J}=6.0 \mathrm{~Hz}, 2 \mathrm{H}), 7.04(\mathrm{t}, \mathrm{J}=8.4 \mathrm{~Hz}, 1 \mathrm{H}), 6.70-6.68(\mathrm{~m}$, $3 \mathrm{H}), 6.63$ (t, J = 4.8 Hz, 1H), 3.47 (brs, 2H), 2.19 (s, 3H).

${ }^{13} \mathbf{C ~ N M R}\left(\mathrm{CDCl}_{3}, 100 \mathrm{MHz}\right): \delta 161.9,158.7,144.2,137.0,128.0,123.2,120.6,114.1,111.9$, 18.5.

IR (neat) cm $\mathrm{cm}^{-1}: 3326 \mathrm{w}, 3211 \mathrm{w}, 3038 \mathrm{w}, 2962 \mathrm{w}, 2921 \mathrm{w}, 2359$ w, 2337 w, $1583 \mathrm{~s}, 1516 \mathrm{~m}$, 1485 w, 1470 w, 1447 m, 1408 m, 1312 w, 1263 w, 1235 w, 1078 w, 993 w, 949w, 901 w, 799 w, $775 \mathrm{~m}, 708 \mathrm{w}$.

HRMS (EI) m/z: [M] $]^{+}$Calcd for $\mathrm{C}_{17} \mathrm{H}_{18} \mathrm{~N}_{4}$ : 200.1062; Found: 200.1059 . 
9.2 General Procedure for the Deprotection of the Pyrimidinyl Group of 2-(4chlorophenyl)-1-(pyrimidin-2-yl)-1H-benzo[d]imidazole (5bd) to Form 2-(4chlorophenyl)-1H-benzo[d]imidazole (9a) (Scheme 10)

Following a previously reported procedure, ${ }^{7} \mathbf{5 b d}(14 \mathrm{mg}, 0.046 \mathrm{mmol})$ was placed in a $5 \mathrm{~mL}$ microwave vial, $1 \mathrm{~mL}$ of aqueous $\mathrm{HCl}$ was added and the mixture was reacted at $150{ }^{\circ} \mathrm{C}(40$ $\mathrm{W})$ for $4 \mathrm{~h}$ in a microwave oven. The reaction mixture was allowed to cool to room temperature and added to EtOAc $(50 \mathrm{~mL})$. A saturated solution of $\mathrm{NaHCO}_{3}$ was then added until the $\mathrm{pH}$ reached 7. The aqueous layer was extracted with EtOAc $(3 \times 50 \mathrm{~mL})$, the combined organic layers were dried over $\mathrm{MgSO}_{4}$ and concentrated in vacuo. The residue was purified by silica gel column chromatography on silica gel (eluent: petroleum ether/EtOAc $=3: 1)$ to give $9 \mathbf{a}(8$ $\mathrm{mg}, 76 \%$ ) as white solid.<smiles>Clc1ccc(-c2nc3ccccc3[nH]2)cc1</smiles>

\section{2-(4-chlorophenyl)-1H-benzo[d]imidazole (9a)}

$76 \%$ yield. $\mathrm{Rf}=0.2$ (eluent: Petroleum ether/EtOAc $=3 / 1$ ). White solid. $\mathrm{Mp}=212-213^{\circ} \mathrm{C}$.

${ }^{1} \mathbf{H}$ NMR (CD $\left.3 \mathrm{OD}, 400 \mathrm{MHz}\right): \delta 8.06(\mathrm{~d}, \mathrm{~J}=8.6 \mathrm{~Hz}, 2 \mathrm{H}), 7.60-7.54(\mathrm{~m}, 4 \mathrm{H}), 7.27(\mathrm{dq}, \mathrm{J}=5.9$, $3.3 \mathrm{~Hz}, 2 \mathrm{H})$.

${ }^{13}$ C NMR (CD 3 OD, $\left.100 \mathrm{MHz}\right): \delta 152.3,137.4,130.5,129.8,129.4$.

IR (neat) cm $\mathrm{cm}^{-1}: 2996$ w, 2969 w, 2947 w, 2360 s, 2339 s, 1740 s, 1471 w, 1448 w, 1428 w, 1369 m, $1272 \mathrm{w}, 1230 \mathrm{~m}, 1216 \mathrm{~m}, 931 \mathrm{w}, 745 \mathrm{w}, 671 \mathrm{w}$.

HRMS (EI) m/z: [M] $]^{+}$Calcd for $\mathrm{C}_{13} \mathrm{H}_{9} \mathrm{~N}_{2} \mathrm{Cl}$ : 228.0454; Found: 228.0453.<smiles>Brc1ccc(-c2nc3ccccc3[nH]2)cc1</smiles>

\section{2-(4-bromophenyl)-1H-benzo[d]imidazole (9b)}

This compound was prepared by reacting $5 \mathbf{b e}(15 \mathrm{mg}, 0.0427 \mathrm{mmol})$ and $1 \mathrm{~mL}$ of aqueous $\mathrm{HCl}$. Purification by column chromatography on silica gel (eluent: petroleum ether/EtOAc: $1 / 1, \mathrm{R}_{\mathrm{f}}$ $=0.5)$ yielded $9 \mathbf{b}(9 \mathrm{mg}, 77 \%)$ as a white yellow solid

$\mathrm{Mp}=254-255^{\circ} \mathrm{C}$.

${ }^{1} \mathbf{H}$ NMR (CD $\left.{ }_{3} \mathrm{OD}, 400 \mathrm{MHz}\right): \delta 7.99(\mathrm{dt}, \mathrm{J}=8.6,1.9 \mathrm{~Hz}, 2 \mathrm{H}), 7.71(\mathrm{dt}, \mathrm{J}=8.5,2.0 \mathrm{~Hz}, 2 \mathrm{H})$, 7.61 (ddd, J = 7.9, 4.7, $1.3 \mathrm{~Hz}, 2 \mathrm{H}), 7.30-7.26$ (m, 2H).

${ }^{13} \mathbf{C ~ N M R}\left(\mathrm{CDCl}_{3}, 100 \mathrm{MHz}\right): \delta 152.3,133.5,130.2,129.6,125.1,124.3$. 
IR (neat) cm $\mathrm{cm}^{-1}: 3025$ w, 2969 w, 2947 w, 2360 s, 2339 s, 1739 s, 1591 w, 1508 w, 1489 w, 1471 w, 1447 w, 1428 m, 1367 m, 1316 w, 1278 w, 1227 m, 1216 m, 1071 w, 1010 w, 832 w, $743 \mathrm{~m}, 713 \mathrm{w}, 680 \mathrm{w}$.

HRMS (EI) m/z: [M] ${ }^{+}$Calcd for $\mathrm{C}_{13} \mathrm{H}_{9} \mathrm{~N}_{2} \mathrm{Br}: 271.9949$; Found: 271.9950 .<smiles>Cc1cccc(-c2nc3ccccc3[nH]2)c1</smiles>

\section{2-(m-tolyl)-1H-benzo[d]imidazole (9c)}

This compound was prepared by reacting $\mathbf{5 b h}(40 \mathrm{mg}, 0.139 \mathrm{mmol})$ and $1 \mathrm{~mL}$ of aqueous $\mathrm{HCl}$. Purification by column chromatography on silica gel (eluent: petroleum ether/EtOAc: $3 / 1, \mathrm{R}_{\mathrm{f}}$ $=0.19)$ yielded $9 \mathrm{c}(27 \mathrm{mg}, 92 \%)$ as a white solid $\mathrm{Mp}=216-217^{\circ} \mathrm{C}$.

${ }^{1} \mathbf{H}$ NMR (CD $\left.3 \mathrm{OD}, 400 \mathrm{MHz}\right): \delta 7.91(\mathrm{~s}, 1 \mathrm{H}), 7.86(\mathrm{~d}, \mathrm{~J}=7.8,1 \mathrm{H}), 7.58$ (brs, 2H), 7.39 (t, J = 7.6, 1H), 7.30 (d, J = 7.6 Hz, 1H), 7.24 (dq, J = 6.1, 4.16 Hz, 2H, 2.43 (s, 3H).

${ }^{13} \mathrm{C}$ NMR (CD $\left.3 \mathrm{OD}, 100 \mathrm{MHz}\right): \delta 153.6,140.2,132.2,131.0,130.2,128.4,125.0,124.0,21.6$. IR (neat) cm $\mathrm{cm}^{-1}: 3051$ w, 2992 w, 2970 w, 2921 w, 2879 w, 2790 w, 2361 s, 2339 s, 1740 s, 1590 w, 1538 w, 1486 w, 1446 m, 1398 w, 1366 m, 1315 w, 1274 w, 1230 s, 1109 w, 975 w, $916 \mathrm{w}, 794 \mathrm{w}, 742 \mathrm{~s}, 687 \mathrm{w}, 972 \mathrm{w}$.

HRMS (EI) m/z: [M] Calcd for $\mathrm{C}_{14} \mathrm{H}_{12} \mathrm{~N}_{2}$ : 200.1000; Found: 200.1001 .

\subsection{General Procedure for Deprotection of Amide and Pyrimidinyl Group of $\mathrm{N}$-(2-Pentyl-} 1-(pyrimidin-2-yl)-1H-benzo[d]imidazol-7-yl)hexanamide (6bk) to Form 2-Pentyl-1Hbenzo[d]imidazol-7-amine (10) (Scheme 10)

Following a previously reported procedure, ${ }^{7}$ 6bk $(23 \mathrm{mg}, 0.061 \mathrm{mmol})$ was placed in a $5 \mathrm{~mL}$ microwave vial, $1 \mathrm{~mL}$ of aqueous $\mathrm{HCl}$ was added and the mixture was reacted at $150{ }^{\circ} \mathrm{C}(40$ $\mathrm{W})$ for $4 \mathrm{~h}$ in a microwave oven. The reaction mixture was allowed to cool to room temperature and added to EtOAc $(50 \mathrm{~mL})$. A saturated solution of $\mathrm{NaHCO}_{3}$ was then added until the $\mathrm{pH}$ reached 7. The aqueous layer was extracted with EtOAc $(3 \times 50 \mathrm{~mL})$, the combined organic layers were dried over $\mathrm{MgSO}_{4}$ and concentrated in vacuo. The residue was purified by silica gel column chromatography om silica gel (eluent: petroleum ether/EtOAc $=1: 2)$ to give $\mathbf{1 0}(4$ $\mathrm{mg}, 33 \%)$ as an oily liquid. 


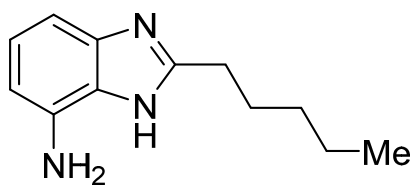

${ }^{1} \mathbf{H}$ NMR (CD $\left.3 \mathrm{OD}, 400 \mathrm{MHz}\right): \delta 6.95(\mathrm{t}, \mathrm{J}=7.9 \mathrm{~Hz}, 1 \mathrm{H}), 6.81(\mathrm{dd}, \mathrm{J}=8.0,0.8 \mathrm{~Hz}, 1 \mathrm{H}), 6.51$ $(\mathrm{dd}, \mathrm{J}=7.8,0.9 \mathrm{~Hz}, 1 \mathrm{H}), 2.86(\mathrm{t}, \mathrm{J}=7.4 \mathrm{~Hz}, 2 \mathrm{H}), 1.83$ (quin, $\mathrm{J}=15.0,7.6 \mathrm{~Hz}, 2 \mathrm{H}$ ), 1.42-1.35 $(\mathrm{m}, 4 \mathrm{H}), 0.93(\mathrm{t}, \mathrm{J}=7.0 \mathrm{~Hz}, 3 \mathrm{H})$.

${ }^{13} \mathbf{C}$ NMR (CD $\left.{ }_{3} \mathrm{OD}, 100 \mathrm{MHz}\right): \delta 154.9,138.0,124.4,108.0,103.3,32.7,29.8,29.3,23.5,14.4$. IR (neat) cm $\mathrm{cm}^{-1}: 3344$ w, 3190 w, 3121 w, 3051 w, 2955 s, 2929 s, 2859 m, 1620 s, 1579 w, 1537 w, 1504 w, 1424 s, 1381 w, 1294 w, 1266 w, 1198 w, 797 w, 783 m, 737 s.

GC-MS: $\mathrm{m} / \mathrm{z}=203.15$.

\section{Control Experiments}

\subsection{Procedure for Reaction of $5 \mathrm{bj}$ with $2 \mathrm{j}$ (Scheme 5)}

To an oven dried screw cap tube equipped with magnetic stir bar in a glove box were added 2methyl-1-(pyrimidin-2-yl)-1H-benzo[d]imidazole (5bj) (31.5 mg, $0.15 \mathrm{mmol}$ ), 3-methyl1,4,2-dioxazol-5-one (2j) (30.1 mg, $0.3 \mathrm{mmol}$ ), [Cp*RhCl2]2 (4.64 mg, $0.0075 \mathrm{mmol}), \mathrm{AgSbF}_{6}$ (15.2 mg, $0.045 \mathrm{mmol}$ ), and $\mathrm{CF}_{3} \mathrm{CH}_{2} \mathrm{OH}(1 \mathrm{~mL})$. The resulting reaction mixture was stirred at $120{ }^{\circ} \mathrm{C}$ for $18 \mathrm{~h}$ on aluminum heating block (Thermo Mighty Stirrer HHE-19G-USIII (KPI)).

\subsection{External Additive Experiment}

Procedure for the TEMPO Addition Experiment (Scheme 6a): To an oven dried screw cap tube equipped with magnetic stir bar in a glove box were added $N$-(o-tolyl)pyrimidin-2-amine (1a) (27.7 mg, $0.15 \mathrm{mmol})$, 3-phenyl-1,4,2-dioxazol-5-one2a (48.9 mg, $0.3 \mathrm{mmol}$ ), $\left[\mathrm{Cp}^{*} \mathrm{RhCl}_{2}\right]_{2}(4.64 \mathrm{mg}, 0.0075 \mathrm{mmol}), \mathrm{AgSbF}_{6}(5.15 \mathrm{mg}, 0.015 \mathrm{mmol})$, TEMPO (23.4 mg, 0.15 mmol) and HFIP $(1 \mathrm{~mL})$. The resulting reaction mixture was stirred at $100{ }^{\circ} \mathrm{C}$ on aluminum heating block (Thermo Mighty Stirrer HHE-19G-USIII (KPI)). After 8 h, the reaction mixture was allowed to cool to room temperature and the resulting mixture was filtered through a celite pad, and the organic phase was concentrated in vacuo. The residue was purified by column chromatography on silica gel (eluent: petroleum ether/EtOAc=2/1) to afford the desired product 3aa (35 mg, 77\%) as a white solid. 


\subsection{Procedure for Competition Experiment (Scheme 6b)}

To an oven dried screw capped tube equipped with magnetic stir bar in a glove box were added $\mathrm{N}$-(2-methoxyphenyl)pyrimidin-2-amine (1c) (30.18 $\mathrm{mg}, \quad 0.15 \quad \mathrm{mmol}), \quad \mathrm{N}$-(2(trifluoromethyl)phenyl)pyrimidin-2-amine (1f) (35.9 mg, $0.15 \mathrm{mmol}$ ), 3-phenyl-1,4,2dioxazol-5-one (2a) (48.9 mg, $0.3 \mathrm{mmol}$ ), [Cp* $\left.\mathrm{RhCl}_{2}\right]_{2}$ (4.64 mg, $\left.0.0075 \mathrm{mmol}\right), \mathrm{AgSbF}_{6}(5.15$ $\mathrm{mg}, 0.015 \mathrm{mmol})$ and HFIP $(1 \mathrm{~mL})$ (Scheme $4 \mathrm{~b})$. The resulting reaction mixture was stirred at $100{ }^{\circ} \mathrm{C}$ on aluminum heating block (Thermo Mighty Stirrer HHE-19G-USIII (KPI)). After $8 \mathrm{~h}$, the reaction mixture was allowed to cool to room temperature, the resulting mixture was filtered through a celite pad and the organic phase was concentrated in vacuo. The residue was purified by column chromatography on silica gel (eluent: petroleum ether/EtOAc $=1 / 1$ ) to afford the desired products $\mathbf{3 c a}(46 \mathrm{mg}, 96 \%)$ and $\mathbf{3 f a}(3 \mathrm{mg}, 5 \%)$.

\subsection{Procedure for Kinetic Isotope Effect Experiments (Scheme 6c)}

To an oven dried screw cap tube equipped with a magnetic stir bar in a glove box were added $N$-(o-tolyl)pyrimidin-2-amine (1a) $(27.7 \mathrm{mg}, 0.15 \mathrm{mmol}$ ) or $N$-(o-tolyl)pyrimidin-2-amine[D]$1 \mathrm{a}$ (27.9 mg, $0.15 \mathrm{mmol}$ ), 3-phenyl-1,4,2-dioxazol-5-one(2a) (48.9 mg, 0.3 mmol), $\left[\mathrm{Cp}^{*} \mathrm{RhCl}_{2}\right]_{2}(4.64 \mathrm{mg}, 0.0075 \mathrm{mmol}), \mathrm{AgSbF}_{6}(5.15 \mathrm{mg}, 0.015 \mathrm{mmol})$ and HFIP $(1 \mathrm{~mL})$. An internal standard, 1,3,5-trimethoxybenzene $(25.22 \mathrm{mg}, 0.15 \mathrm{mmol})$ was added and the reaction mixture was then stirred at $100{ }^{\circ} \mathrm{C}$ on aluminum heating block (Thermo Mighty Stirrer HHE19G-USIII (KPI)). At regular intervals (10, 20, 30, 40, 50, 60, 70, 80, 90, and $100 \mathrm{~min})$, the reaction vessel was cooled to room temperature, introduced to the glove box and an aliquot withdrawn and placed in an NMR tube. The solvent was evaporated under reduced pressure, and subjected to NMR analysis in $\mathrm{CDCl}_{3}$. The concentration of the product 3aa obtained in each sample was determined by comparing the signal strength to that for the internal standard, trimethoxybenzene. The data for the concentration of the product $v s$ time ( $\mathrm{min}$ ) plot was plotted using MS-excel. For the reaction rate, the data were fitted linear with MS-excel and the rate was determined by the initial rate method (up to 100 minutes). The slope of the linear fitting represents the reaction rate. The initial rate obtained for the coupling of 1a with oxazolone 2 was5.7 $\times 10^{-4} \mathrm{Mmin}^{-1}$, whereas the rate for the coupling of $N$-(2-methylphenyl-6-d)pyrimidin2-amine [D]-2a with oxazolone 2 was6.1 $10^{-4} \mathrm{Mmin}^{-1}$. Thus, the $k_{\mathrm{H}} / k_{\mathrm{D}}=5.7 \times 10^{-4} / 6.1 \times 10^{-4}=$ 0.93 . 


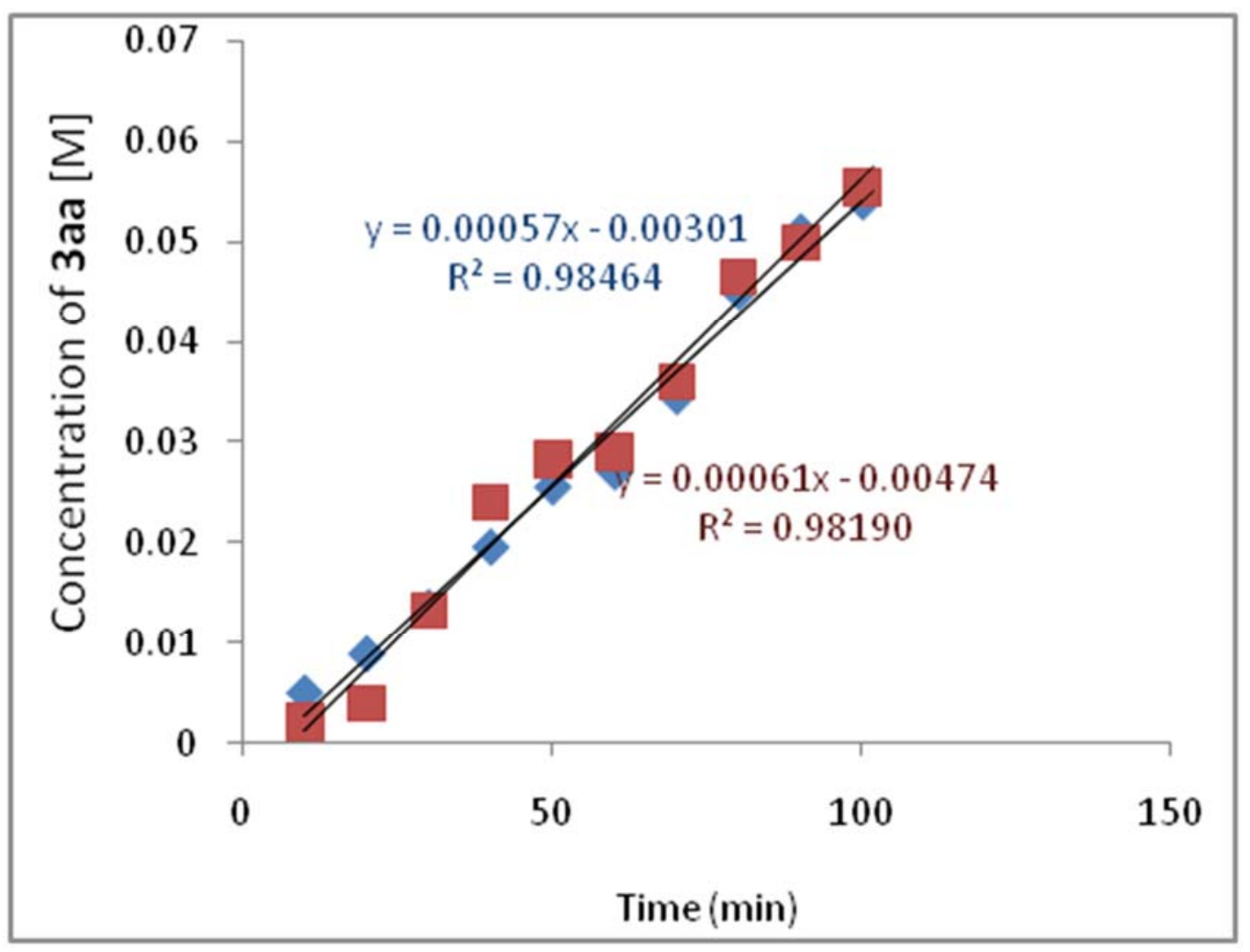

Figure 1. Time dependent formation of 3aa in the reaction of $\mathbf{1 a}$ and [D]-1a with $\mathbf{2 a}$.

\subsection{Procedure for H/D Scrambling Experiment (Scheme 6d)}

To an oven dried screw cap tube equipped with magnetic stir bar on a glove box, were added $\mathrm{N}$-(o-tolyl)pyrimidin-2-amine (1a) $\left(27.7 \mathrm{mg}, 0.15 \mathrm{mmol} \text {, [Cp* } \mathrm{RhCl}_{2}\right]_{2}$ (4.64 mg, $0.0075 \mathrm{mmol}$ ), $\mathrm{AgSbF}_{6}(5.15 \mathrm{mg}, 0.015 \mathrm{mmol}), \mathrm{HFIP}(1 \mathrm{~mL})$ and $\mathrm{CD}_{3} \mathrm{OD}(0.2 \mathrm{~mL})$. The reaction mixture was reacted at $100{ }^{\circ} \mathrm{C}$ for $8 \mathrm{~h}$ on aluminum heating block (Thermo Mighty Stirrer HHE-19G-USIII (KPI)). The reaction mixture was then allowed to cool to room temperature and the resulting mixture was filtered through a celite pad and the organic phase was then concentrated in vacuo. The residue was purified by column chromatography on silica gel (eluent: petroleum ether/EtOAc $=5 / 1)$ to afford the desired products $1 \mathbf{a}(23 \mathrm{mg}, 83 \%)$. The deuterium incorporation was determined to be $36 \%$ based on ${ }^{1} \mathrm{H}-\mathrm{NMR}$ spectral measurements. 


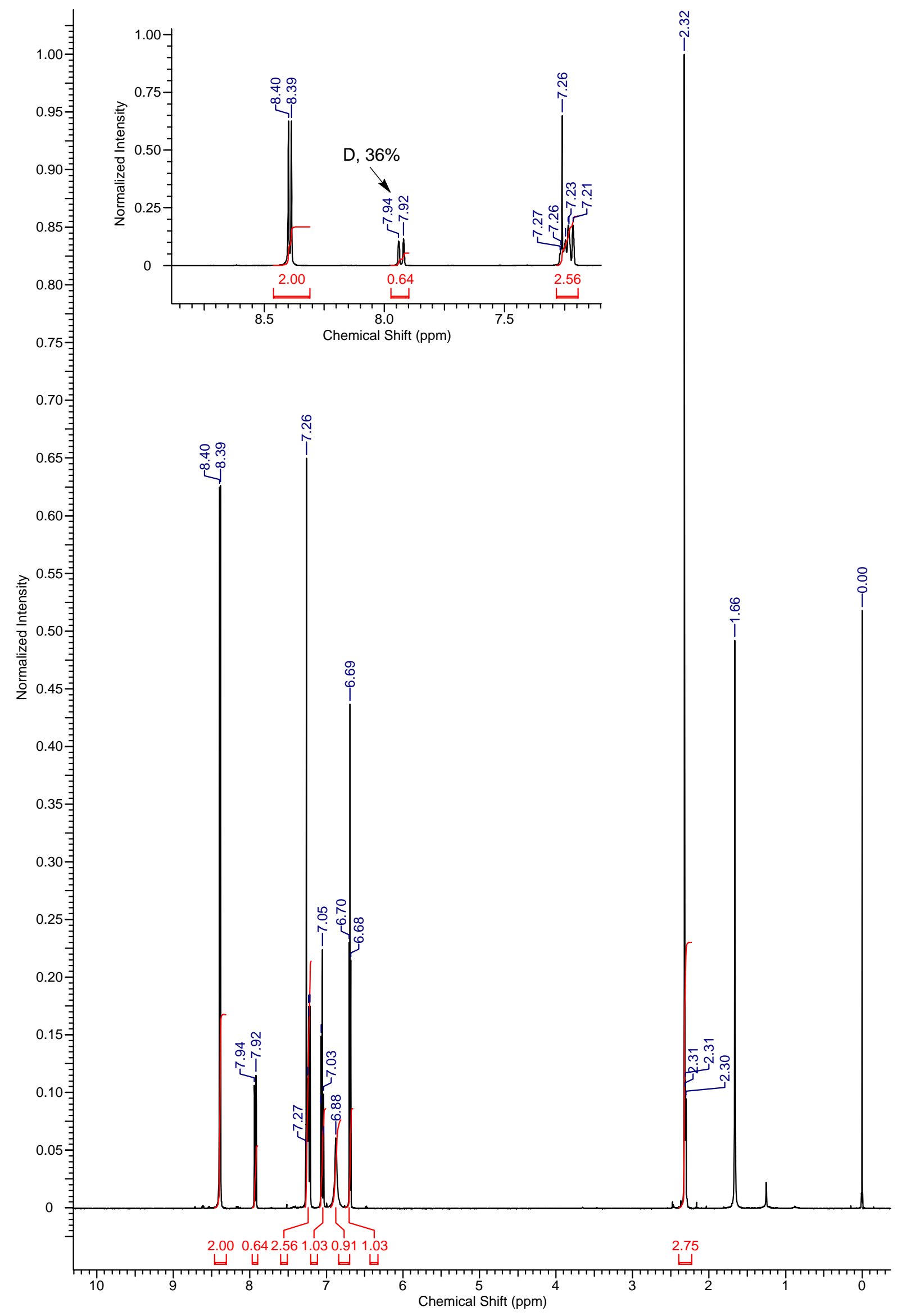




\section{X-ray Crystalllographic Structure Analysis:}

i)

\section{Data of compound 3aa}

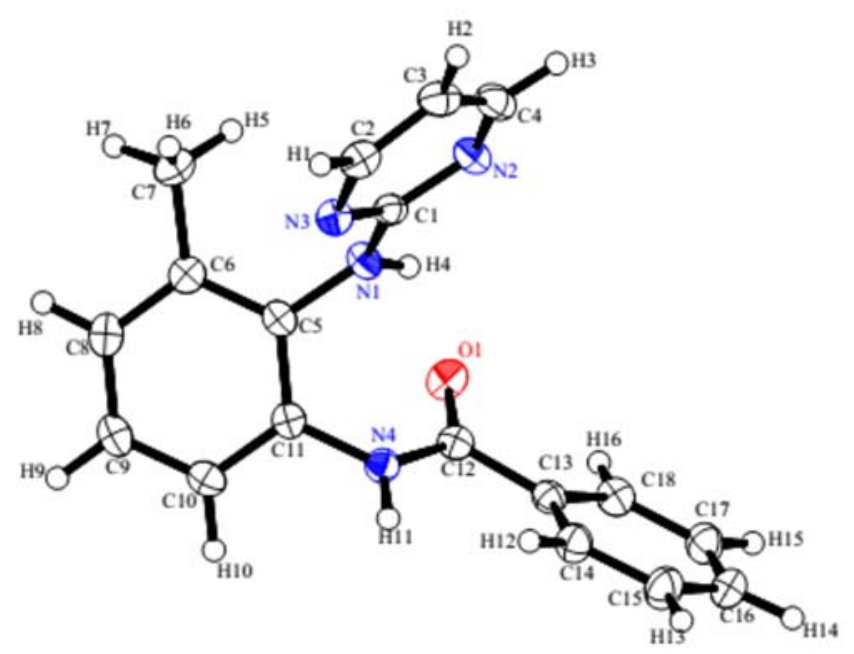

Plots are drawn at 50\% probability level.

CCDC-1983469 contains the supplementary crystallographic data of compound 3aa. The crystals of 3aa were obtained by dissolving 3aa in acetone followed by slow evaporation of acetone. X-Ray crystallographic structure analysis of $\mathrm{N}$-(3-methyl-2-(pyrimidin-2ylamino)phenyl)benzamide 3aa was performed on Rigaku XtaLAB P200 diffractometer using multi-layer mirror monochromated Mo-Ka radiation. The data were collected at a temperature of $-150 \pm 1{ }^{\circ} \mathrm{C}$ to a maximum $2 \theta$ value of $62.1^{\circ}$. A total of 674 oscillation images were collected. A sweep of data was done using $\omega$ scans from -8.0 to $18.0^{\circ}$ in $0.50^{\circ}$ step, at $\chi=-19.0^{\circ}$ and $\phi=$ $-60.0^{\circ}$.The structure was solved by direct method (SIR92) ${ }^{8}$ and expanded using Fourier techniques. The non-hydrogen atoms were refined anisotropically. Hydrogen atoms were refined isotropically. The crystal data are mentioned below:

Note: PLAT029_ALERT_3_A_diffrn_measured_fraction_theta full value Low. 


\section{Crystal data:}

Empirical Formula

Formula Weight

Crystal Color, Habit

Crystal Dimensions

Crystal System

Lattice Type

Lattice Parameters
$\mathrm{C}_{18} \mathrm{H}_{16} \mathrm{~N}_{4} \mathrm{O}$

304.35

colorless, prism

$0.400 \times 0.300 \times 0.200 \mathrm{~mm}$

triclinic

Primitive

$$
\begin{aligned}
& \mathrm{a}=9.2374(4) \AA \\
& \mathrm{b}=9.3002(7) \AA \\
& \mathrm{c}=9.8658(6) \AA \\
& \mathrm{a}=112.890(7) \mathrm{o} \\
& \mathrm{b}=92.503(4)^{\mathrm{o}} \\
& \mathrm{g}=102.235(5)^{\mathrm{o}} \\
& \mathrm{V}=755.57(9) \AA^{3}
\end{aligned}
$$

Space Group

P-1 (\#2)

$\mathrm{Z}$ value

2

$D_{\text {calc }}$

$1.338 \mathrm{~g} / \mathrm{cm}^{3}$

$\mathrm{F}_{000}$

320.00

$0.868 \mathrm{~cm}^{-1}$

$\mu(\operatorname{MoK} \alpha)$ 


\section{ii) Data of compound $4 \mathrm{ba}$}

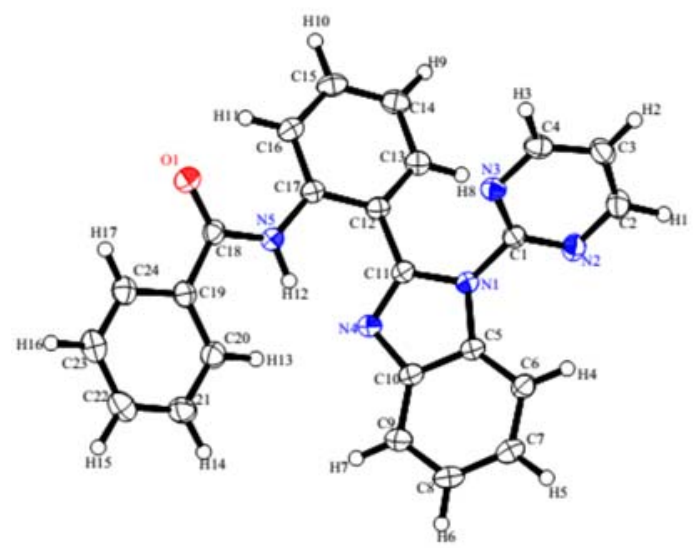

Plots are drawn at 50\% probability level.

CCDC-1983470 contains the supplementary crystallographic data of compound $\mathbf{4 b a}$. The crystals of $\mathbf{4 b a}$ were obtained by dissolving $\mathbf{4 b a}$ in dichloromethane followed by slow evaporation of dichloromethane. X-Ray crystallographic structure analysis of $\mathrm{N}$-(2-(1(pyrimidin-2-yl)-1H-benzo[d]imidazol-2-yl)phenyl)benzamide 4 ba was performed on Rigaku XtaLAB P200 diffractometer using multi-layer mirror monochromated Mo-Ka radiation. The data were collected at a temperature of $-150 \pm 1{ }^{\circ} \mathrm{C}$ to a maximum $2 \theta$ value of $62.4^{\circ}$. A total of 838 oscillation images were collected. A sweep of data was done using $\omega$ scans from -44.0 to $30.0^{\circ}$ in $0.50^{\circ}$ step, at $\chi=-87.0^{\circ}$ and $\phi=-75.0^{\circ}$. The structure was solved by direct method $(\mathrm{SIR} 92)^{8}$ and expanded using Fourier techniques. The non-hydrogen atoms were refined anisotropically. Hydrogen atoms were refined isotropically. The crystal data are mentioned below:

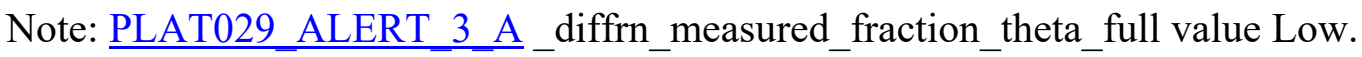




\section{Crystal data:}

Empirical Formula

$\mathrm{C}_{24} \mathrm{H}_{17} \mathrm{~N}_{5} \mathrm{O}$

Formula Weight

391.43

Crystal Color, Habit

colorless, prism

Crystal Dimensions

$0.400 \times 0.300 \times 0.200 \mathrm{~mm}$

Crystal System

Lattice Type

Lattice Parameters

Space Group

triclinic

Primitive

$$
\mathrm{a}=9.4659(11) \AA
$$

$\mathrm{b}=10.9496(10) \AA$

$\mathrm{c}=11.4291(11) \AA$

$\mathrm{a}=65.681(9)^{\mathrm{o}}$

$\mathrm{b}=68.636(10)^{\mathrm{o}}$

$\mathrm{g}=64.508(10)^{\mathrm{o}}$

$\mathrm{V}=948.87(19) \AA^{3}$

$\mathrm{Z}$ value

P-1 (\#2)

Dcalc

$1.370 \mathrm{~g} / \mathrm{cm}^{3}$

$\mathrm{F}_{000}$

408.00

$\mu(\mathrm{MoK} \alpha) \quad 0.879 \mathrm{~cm}^{-1}$ 


\section{iii) Data of compound 3da}

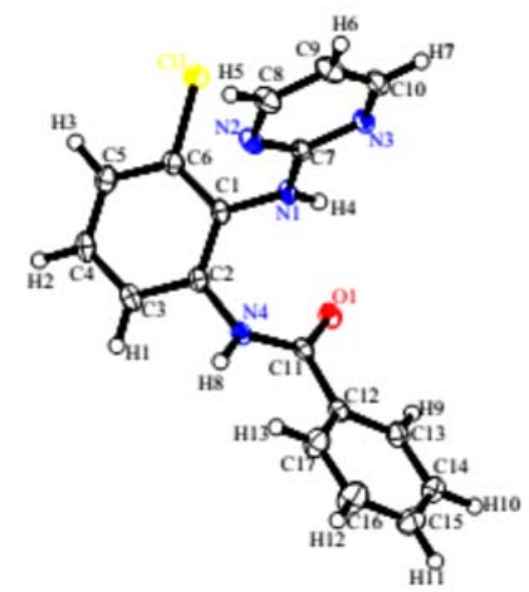

Plots are drawn at 50\% probability level.

CCDC-1983471 contains the supplementary crystallographic data of compound 3da. The crystals of 3da were obtained by dissolving 3da in dichloromethane followed by slow evaporation of dichloromethane. X-Ray crystallographic structure analysis of $N$-(3-chloro-2(pyrimidin-2-ylamino)phenyl)benzamide3da was performed on Rigaku XtaLAB P200 diffractometer using multi-layer mirror monochromated $\mathrm{Cu}-\mathrm{K} \alpha$ radiation. The data were collected at a temperature of $-150 \pm 1^{\circ} \mathrm{C}$ to a maximum $2 \theta$ value of $136.0^{\circ}$. A total of 4548 oscillation images were collected. A sweep of data was done using $\omega$ scans from -14.0 to $38.0^{\circ}$ in $0.50^{\circ}$ step, at $\chi=85.0^{\circ}$ and $\phi=-23.0^{\circ}$. The structure was solved by direct method (SIR92) ${ }^{8}$ and expanded using Fourier techniques. The non-hydrogen atoms were refined anisotropically. Hydrogen atoms were refined isotropically. The crystal data are mentioned below: 


\section{Crsystal data:}

Empirical Formula

Formula Weight

Crystal Color, Habit

Crystal Dimensions

Crystal System

Lattice Type

Lattice Parameters

Space Group

$\mathrm{Z}$ value

Dcalc

$\mathrm{F}_{000}$

$\mu(\mathrm{CuK} \alpha)$
$\mathrm{C}_{17} \mathrm{H}_{13} \mathrm{ClN}_{4} \mathrm{O}$

324.77

colourless, needle

$0.500 \times 0.100 \times 0.100 \mathrm{~mm}$

triclinic

Primitive

$\mathrm{a}=7.9353(5) \AA$

$\mathrm{b}=8.9931(6) \AA$

$c=11.7260(8) \AA$

$a=73.767(6) \mathrm{o}$

$\mathrm{b}=74.312(6)^{\mathrm{o}}$

$\mathrm{g}=69.164(7)^{\mathrm{o}}$

$\mathrm{V}=737.14(9) \AA^{3}$

P-1 (\#2)

2

$1.463 \mathrm{~g} / \mathrm{cm}^{3}$

336.00

$23.808 \mathrm{~cm}^{-1}$ 


\section{iv) Data of compound 3ab}

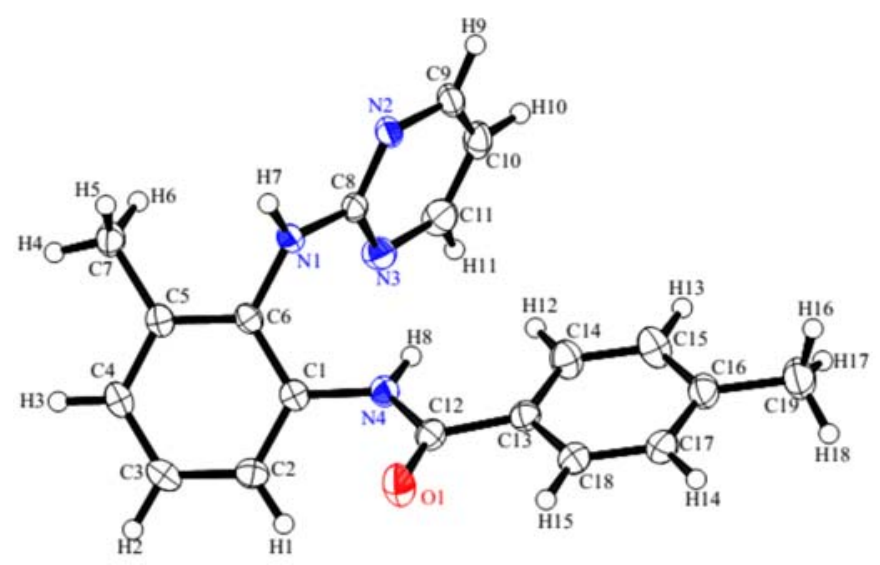

Plots are drawn at 50\% probability level.

CCDC-1961840 contains the supplementary crystallographic data of compound 3ab. The crystals of 3ab were obtained by dissolving 3ab in dichloromethane followed by slow evaporation of dichloromethane. X-Ray crystallographic structure analysis of 4-methyl- $\mathrm{N}-(3-$ methyl-2-(pyrimidin-2-ylamino)phenyl)benzamide 3ab was performed on Rigaku XtaLAB P200 diffractometer using multi-layer mirror monochromated $\mathrm{Cu}-\mathrm{K} \alpha$ radiation. The data were collected at a temperature of $-150 \pm 1^{\circ} \mathrm{C}$ to a maximum $2 \theta$ value of $135.8^{\circ}$. A total of 3062 oscillation images were collected. A sweep of data was done using w scans from 29.0 to $83.0^{\circ}$ in $0.50^{\circ}$ step, at $\chi=48.0^{\circ}$ and $\phi=44.0^{\circ}$. The structure was solved by direct method (SIR92) ${ }^{8}$ and expanded using Fourier techniques. The non-hydrogen atoms were refined anisotropically. Hydrogen atoms were refined isotropically. The crystal data are mentioned below:

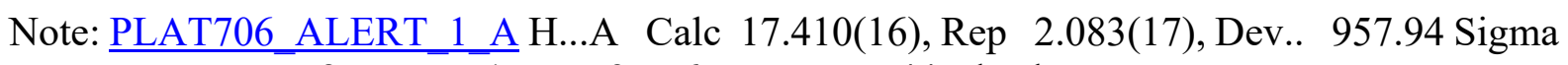
H7 $\quad$-N2 $\quad 1.555 \quad 3.776 \ldots \ldots . . . \quad$ \# 44 Check

PLAT707_ALERT_1_A D...A Calc 17.1875(15), Rep 2.9890(15), Dev.. 9465.67 Sigma N1 $\quad$-N2 $\quad 1.555 \quad 3.776 \ldots \ldots . . . \quad$ \# 44 Check

PLAT708 ALERT 1 A D-H..A Calc 74.4(10), Rep 178.9(15), Dev.. 104.50 Sigma $\begin{array}{lllllll}\mathrm{N} 1 & -\mathrm{H} 7 & -\mathrm{N} 2 & 1.555 & 1.555 & 3.776 \quad \text { \# } 71 \text { Check }\end{array}$ 


\section{Crsystal data:}

Empirical Formula

Formula Weight

Crystal Color, Habit

Crystal Dimensions

Crystal System

Lattice Type

Lattice Parameters

Space Group

$\mathrm{Z}$ value

Dcalc

$\mathrm{F}_{000}$

$\mu(\mathrm{CuK} \alpha)$
$\mathrm{C}_{19} \mathrm{H}_{18} \mathrm{~N}_{4} \mathrm{O}$

318.38

yellow, prism

$0.600 \times 0.500 \times 0.200 \mathrm{~mm}$

monoclinic

Primitive

$\mathrm{a}=13.8180(4) \AA$

$\mathrm{b}=9.0601(3) \AA$

$\mathrm{c}=12.8122(3) \AA$

$\mathrm{b}=94.180(3)^{\mathrm{o}}$

$\mathrm{V}=1599.72(8) \AA^{3}$

P2 1 /c (\#14)

4

$1.322 \mathrm{~g} / \mathrm{cm}^{3}$

672.00

$6.797 \mathrm{~cm}^{-1}$ 


\section{V) Data of compound 5bb:}

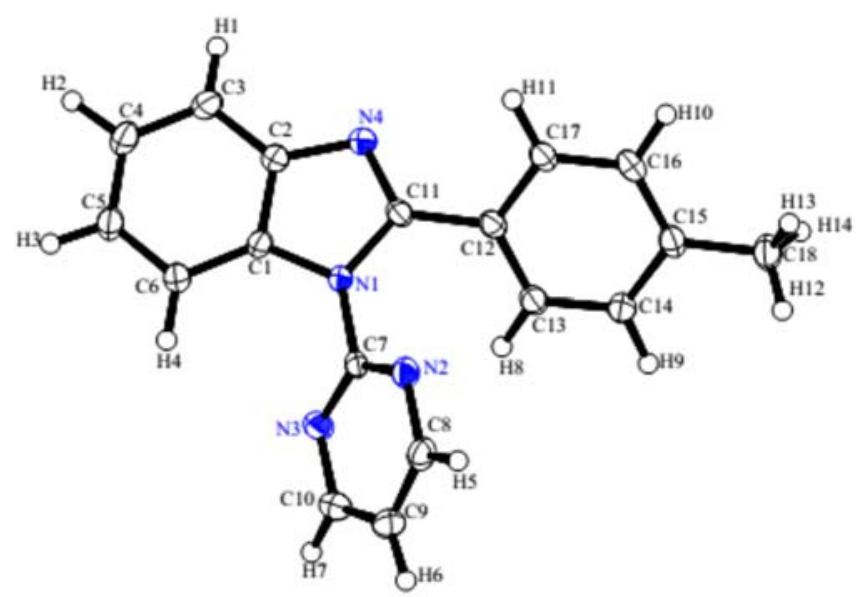

Plots are drawn at 50\% probability level.

CCDC-1983472 contains the supplementary crystallographic data of compound $\mathbf{5 b b}$. The crystals of $\mathbf{5 b b}$ were obtained by dissolving $\mathbf{5 b b}$ in dichloromethane followed by slow evaporation of dichloromethane. X-Ray crystallographic structure analysis of 1-(pyrimidin-2yl)-2-(p-tolyl)-1H-benzo[d]imidazole $\mathbf{5 b b}$ was performed on Rigaku XtaLAB P200 diffractometer using multi-layer mirror monochromated $\mathrm{Cu}-\mathrm{K} \alpha$ radiation. The data were collected at a temperature of $-150 \pm 1{ }^{\circ} \mathrm{C}$ to a maximum $2 \theta$ value of $136.1^{\circ}$. A total of 4838 oscillation images were collected. A sweep of data was done using $\omega$ scans from 13.0 to $101.0^{\circ}$ in $0.50^{\circ}$ step, at $\chi=57.0^{\circ}$ and $\phi=120.0^{\circ}$. The structure was solved by direct method (SIR92) ${ }^{8}$ and expanded using Fourier techniques. The non-hydrogen atoms were refined anisotropically. Hydrogen atoms were refined isotropically. The crystal data are mentioned below: 


\section{Crystal data:}

Empirical Formula

Formula Weight

Crystal Color, Habit

Crystal Dimensions

Crystal System

Lattice Type

Lattice Parameters

Space Group

$\mathrm{Z}$ value

$D_{\text {calc }}$

F000

$\mu(\mathrm{CuK} \alpha) \quad 6.450 \mathrm{~cm}^{-1}$
$\mathrm{C}_{18} \mathrm{H}_{14} \mathrm{~N}_{4}$

286.34

colourless, needle

$0.700 \times 0.200 \times 0.200 \mathrm{~mm}$

triclinic

Primitive

$$
\mathrm{a}=\text { 9.1259(9) } \AA
$$

$\mathrm{b}=9.3808(10) \AA$

$\mathrm{c}=10.0109(12) \AA$

$\mathrm{a}=62.502(12)^{\mathrm{o}}$

$\mathrm{b}=71.364(10)^{\mathrm{o}}$

$\mathrm{g}=83.953(8)^{\mathrm{o}}$

$\mathrm{V}=719.43(16) \AA^{3}$

P-1 (\#2)

2

$1.322 \mathrm{~g} / \mathrm{cm}^{3}$

300.00 


\section{Data of compound 5bc:}

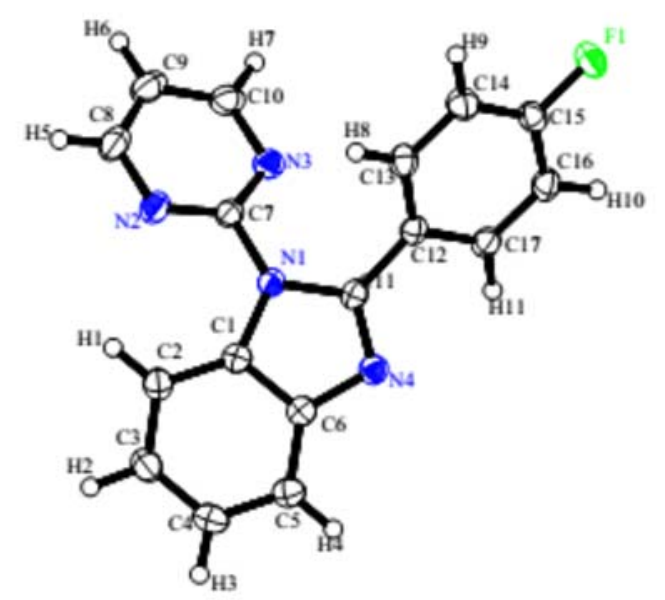

Plots are drawn at 50\% probability level.

CCDC-1983474 contains the supplementary crystallographic data of compound 5bc. The crystals of 5 bc were obtained by dissolving 5 bc in dichloromethane followed by slow evaporation of dichloromethane. X-Ray crystallographic structure analysis of 2-(4fluorophenyl)-1-(pyrimidin-2-yl)-1H-benzo[d]imidazole 5bc was performed on Rigaku XtaLAB P200 diffractometer using multi-layer mirror monochromated $\mathrm{Cu}-\mathrm{K} \alpha$ radiation. The data were collected at a temperature of $-150 \pm 1{ }^{\circ} \mathrm{C}$ to a maximum $2 \theta$ value of $135.6^{\circ}$. A total of 1978 oscillation images were collected. A sweep of data was done using $\omega$ scans from -67.0 to $-6.0^{\circ}$ in $0.50^{\circ}$ step, at $\chi=93.0^{\circ}$ and $\phi=-94.0^{\circ}$. The structure was solved by direct method $(\mathrm{SIR} 92)^{8}$ and expanded using Fourier techniques. The non-hydrogen atoms were refined anisotropically. Hydrogen atoms were refined isotropically. The crystal data are mentioned below: 


\section{Crystal data:}

Empirical Formula

$\mathrm{C}_{17} \mathrm{H}_{11} \mathrm{FN}_{4}$

Formula Weight

290.30

Crystal Color, Habit

colourless, prism

Crystal Dimensions

$0.600 \times 0.600 \times 0.500 \mathrm{~mm}$

Crystal System

orthorhombic

Lattice Type

Lattice Parameters

Space Group

Primitive

$\mathrm{a}=15.4836(7) \AA$

$\mathrm{b}=10.2091(5) \AA$

$\mathrm{c}=17.2959(9) \AA$

$\mathrm{V}=2734.0(2) \AA^{3}$

$Z$ value

Pbca (\#61)

D calc

8

$1.410 \mathrm{~g} / \mathrm{cm}^{3}$

F000

1200.00

$\mu(\mathrm{CuK} \alpha) \quad 7.979 \mathrm{~cm}^{-1}$ 


\section{Data of compound 6bj:}

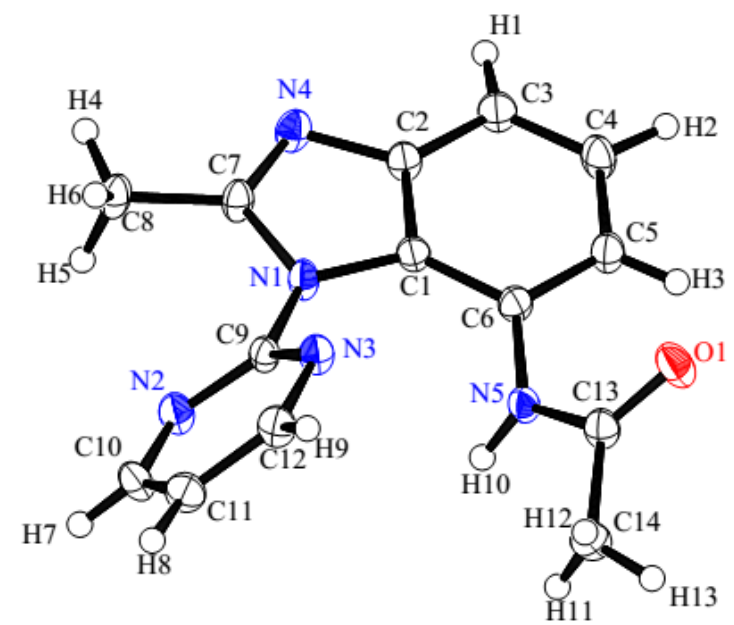

Plots are drawn at 50\% probability level.

CCDC-1983475 contains the supplementary crystallographic data of compound $\mathbf{6 b j}$. The crystals of $\mathbf{6 b j}$ were obtained by dissolving $\mathbf{6 b j}$ in dichloromethane followed by slow evaporation of dichloromethane. X-Ray crystallographic structure analysis of $N$-(2-methyl-1(pyrimidin-2-yl)-1H-benzo[d]imidazol-7-yl)acetamide 6bj was performed on Rigaku XtaLAB P200 diffractometer using multi-layer mirror monochromated $\mathrm{Cu}-\mathrm{K} \alpha$ radiation. The data were collected at a temperature of $-150 \pm 1{ }^{\circ} \mathrm{C}$ to a maximum $2 \theta$ value of $135.8^{\circ}$. A total of 1414 oscillation images were collected. A sweep of data was done using $\omega$ scans from -34.0 to $-6.0^{\circ}$ in $0.50^{\circ}$ step, at $\chi=10.0^{\circ}$ and $\phi=-131.0^{\circ}$. The structure was solved by direct method (SIR92) ${ }^{8}$ and expanded using Fourier techniques. The non-hydrogen atoms were refined anisotropically. Hydrogen atoms were refined isotropically. The crystal data are mentioned below:

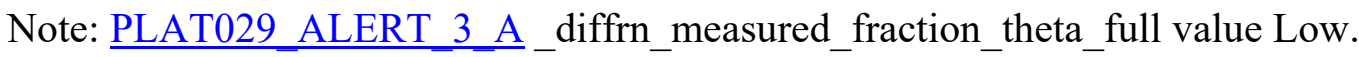




\section{Crystal Data}

Empirical Formula

$\mathrm{C}_{14} \mathrm{H}_{13} \mathrm{~N}_{5} \mathrm{O}$

Formula Weight

267.29

Crystal Color, Habit

colorless, prism

Crystal Dimensions

$0.500 \times 0.200 \times 0.150 \mathrm{~mm}$

Crystal System

monoclinic

Lattice Type

Lattice Parameters

Space Group

Primitive

$\mathrm{a}=3.7962(2) \AA$

$\mathrm{b}=17.2299(9) \AA$

$c=9.5006(5) \AA$

$\mathrm{b}=100.910(6)^{\mathrm{o}}$

$\mathrm{V}=610.18(6) \AA^{3}$

$\mathrm{Z}$ value

P2 1 (\#4)

Dcalc

$1.455 \mathrm{~g} / \mathrm{cm}^{3}$

$\mathrm{F}_{000}$

280.00

$\mu\left(\mathrm{CuK}_{\alpha}\right) \quad 7.992 \mathrm{~cm}^{-1}$ 


\section{Data of compound 8:}

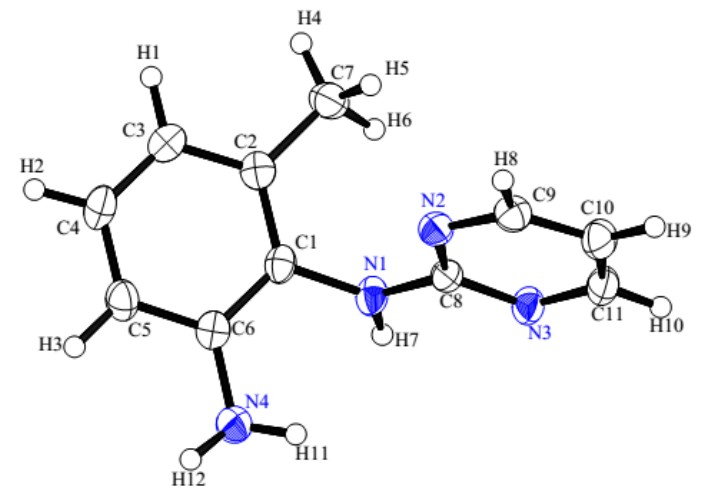

Plots are drawn at 50\% probability level.

CCDC-1983476 contains the supplementary crystallographic data of compound $\mathbf{8}$. The crystals of $\mathbf{8}$ were obtained by dissolving $\mathbf{8}$ in dichloromethane followed by slow evaporation of dichloromethane. X-Ray crystallographic structure analysis of 6-methyl- $N^{1}$-(pyrimidin-2yl)benzene-1,2-diamine 8 was performed on Rigaku XtaLAB P200 diffractometer using multilayer mirror monochromated $\mathrm{Cu}-\mathrm{K} \alpha$ radiation. The data were collected at a temperature of $150 \pm 1^{\circ} \mathrm{C}$ to a maximum $2 \theta$ value of $135.4^{\circ}$. A total of 2974 oscillation images were collected. A sweep of data was done using $\omega$ scans from -49.0 to $-14.0^{\circ}$ in $0.50^{\circ}$ step, at $\chi=-57.0^{\circ}$ and $\phi$ $=-60.0^{\circ}$. The structure was solved by direct method (SIR92) ${ }^{8}$ and expanded using Fourier techniques. The non-hydrogen atoms were refined anisotropically. Hydrogen atoms were refined isotropically. The crystal data are mentioned below: 


\section{Crystal Data}

Empirical Formula

$\mathrm{C}_{11} \mathrm{H}_{12} \mathrm{~N}_{4}$

Formula Weight

200.24

Crystal Color, Habit

lightyellow, prism

Crystal Dimensions

$0.600 \times 0.500 \times 0.500 \mathrm{~mm}$

Crystal System

monoclinic

Lattice Type

Lattice Parameters

Primitive

$\mathrm{a}=11.0378(6) \AA$

$\mathrm{b}=8.1166(6) \AA$

$c=11.5037(8) \AA$

$\mathrm{b}=96.389(5)^{\mathrm{o}}$

$\mathrm{V}=1024.21(12) \AA^{3}$

Space Group

P21/n (\#14)

$\mathrm{Z}$ value

4

$\mathrm{D}_{\text {calc }}$

$1.299 \mathrm{~g} / \mathrm{cm}^{3}$

$\mathrm{F}_{000}$

424.00

$\mu\left(\mathrm{CuK}_{\alpha}\right)$

$6.598 \mathrm{~cm}^{-1}$ 


\section{Data of compound $9 b^{9}$}

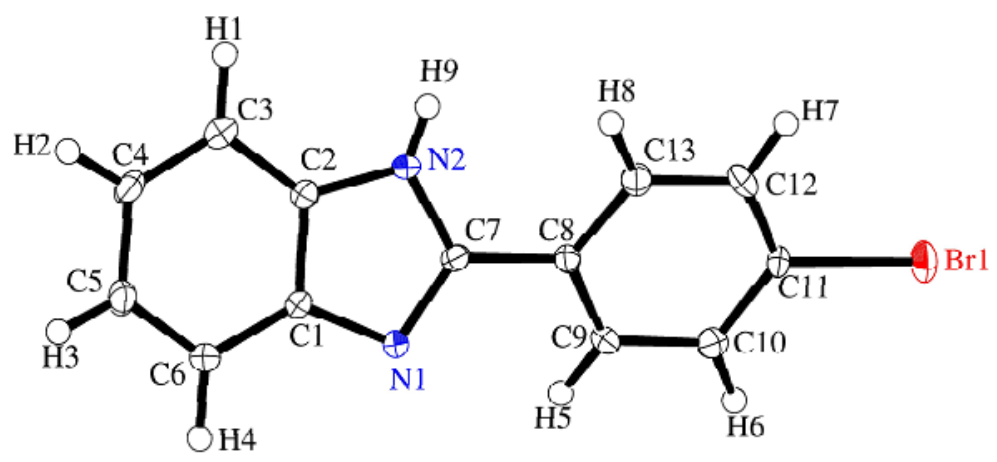

Plots are drawn at 50\% probability level.

CCDC-1984360 contains the supplementary crystallographic data of compound 9b. The crystals of $9 \mathbf{b}$ were obtained by dissolving $9 \mathbf{b}$ in dichloromethane followed by slow evaporation of dichloromethane. X-Ray crystallographic structure analysis of 2-(4-bromophenyl)-1Hbenzo $[d]$ imidazole 9b was performed on Rigaku XtaLAB P200 diffractometer using multilayer mirror monochromated Mo-K $\alpha$ radiation. The data were collected at a temperature of 20 $\pm 1^{\circ} \mathrm{C}$ to a maximum $2 \theta$ value of $61.2^{\circ}$. A total of 374 oscillation images were collected. A sweep of data was done using $\omega$ scans from -25.0 to $0.0^{\circ}$ in $0.50^{\circ}$ step, at $\chi=-35.0^{\circ}$ and $\phi=$ 56.0 $0^{\circ}$.The structure was solved by direct method (SIR92) ${ }^{8}$ and expanded using Fourier techniques. The non-hydrogen atoms were refined anisotropically. Hydrogen atoms were refined isotropically. The crystal data are mentioned below:

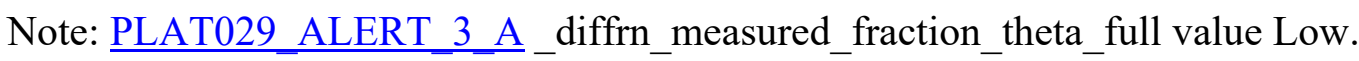


A. Crystal Data

Empirical Formula

Formula Weight

Crystal Color, Habit

Crystal Dimensions

Crystal System

Lattice Type

Lattice Parameters

Space Group

$Z$ value

Dcalc

$\mathrm{F}_{000}$

$\mu(\operatorname{MoK} \alpha)$
$\mathrm{C}_{13} \mathrm{H}_{9} \mathrm{BrN}_{2}$

273.13

colorless, prism

$1.000 \times 0.500 \times 0.200 \mathrm{~mm}$

orthorhombic

Primitive

$\mathrm{a}=9.4129(4) \AA$

$\mathrm{b}=9.7215(5) \AA$

$\mathrm{c}=23.4214(13) \AA$

$\mathrm{V}=2143.23(19) \AA^{3}$

Pbca (\#61)

8

$1.693 \mathrm{~g} / \mathrm{cm}^{3}$

1088.00

$38.164 \mathrm{~cm}^{-1}$ 


\section{References:}

(1) Ackermann, L.; Lygin, A. V. Org. Lett. 2012, 14, 764-767.

(2) Bizet, V.; Buglioni, L.; Bolm, C. Angew. Chem. Int. Ed. 2014, 53, 5639-5642.

(3) Mi, X.; Feng, W.; Pi, C.; Cui, X. J. Org. Chem.2019, 84, 5305-5312.

(4) Shi, H.; Dixon, D. J. Chem. Sci. 2019, 10, 3733-3737.

(5) van Vliet, K. M.; Polak, L. H.; Siegler, M. A.; van der Vlugt, J. I.; Guerra, C. F.; de Bruin, B. J. Am. Chem. Soc. 2019, 141, 15240-15249.

(6) Park, Y.; Park, K. T.; Kim, J. G.; Chang, S. J. Am. Chem. Soc.2015, 137, 4534-4542.

(7) Wang, L.; Yang, Z.; Yang, M.; Zhang, R.; Kuai, C.; Cui, X. Org. Biomol. Chem. 2017, 15, 8302-8307.

(8) Altomare, A.; Cascarano, G.; Giacovazzo, C.; Guagliardi, A. J. Appl. Cryst. 1993, 26, 343-350.

(9) Rashid, N.; Tahir, M. K.; Yusof, N. M.; Yamin, B. M. Acta Crystallogr., Sect. E: Struct. Rep. Online, 2007, 63, o1260-o1261. 
13) ${ }^{1} \mathrm{H}$ and ${ }^{13} \mathrm{C}$ NMR of Compounds

13.1) Spectra of Compound 1
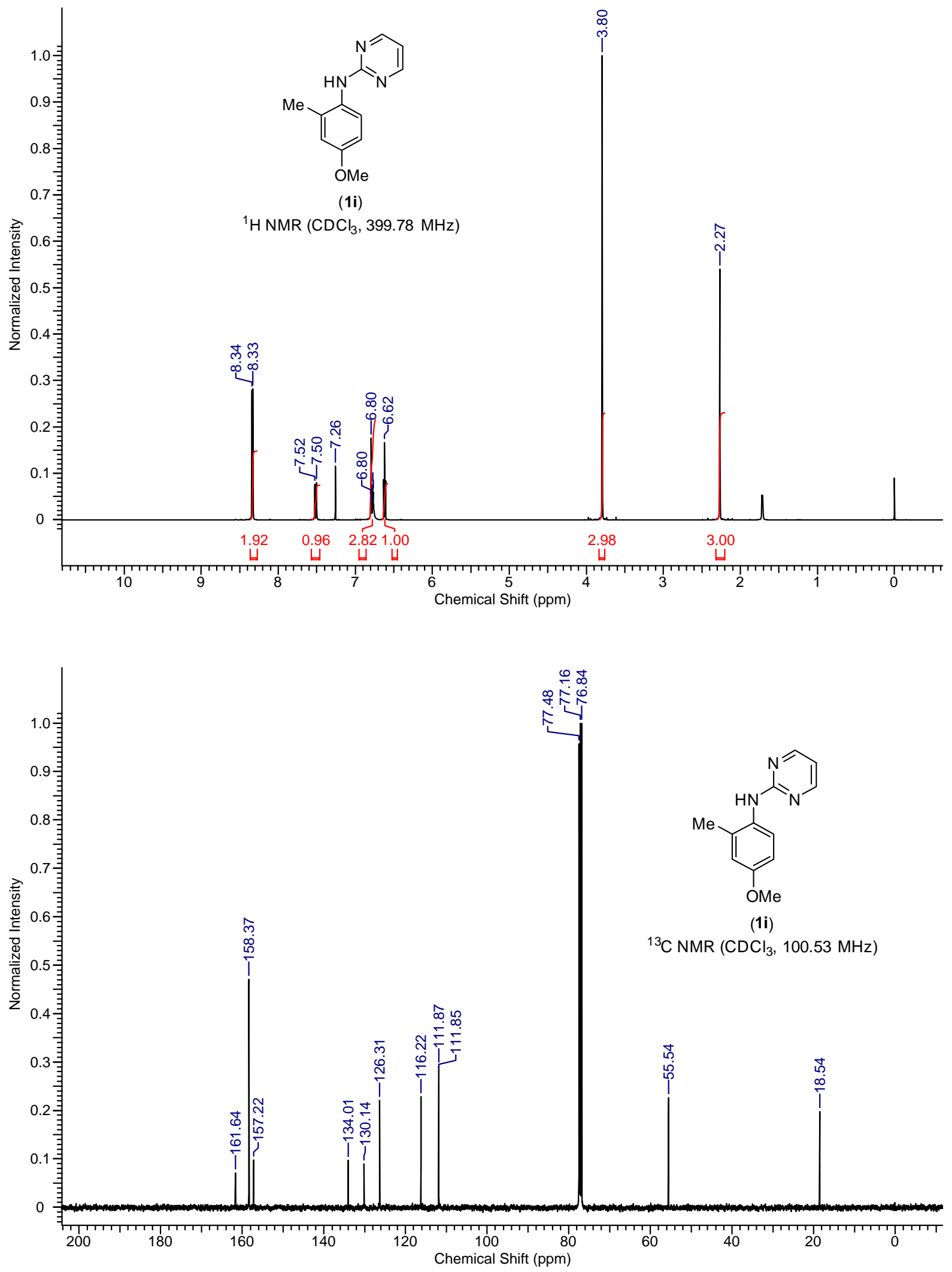

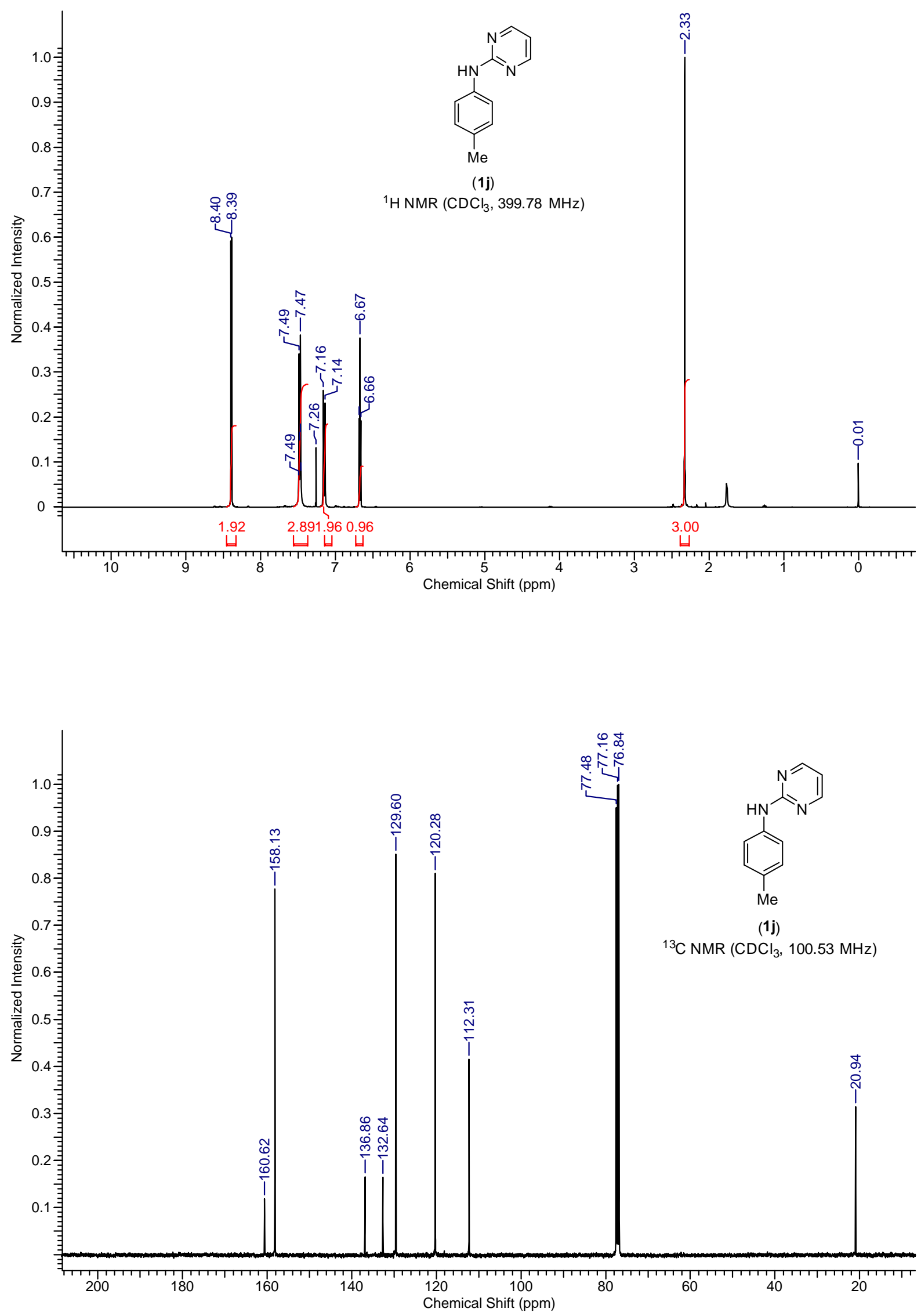

S63 

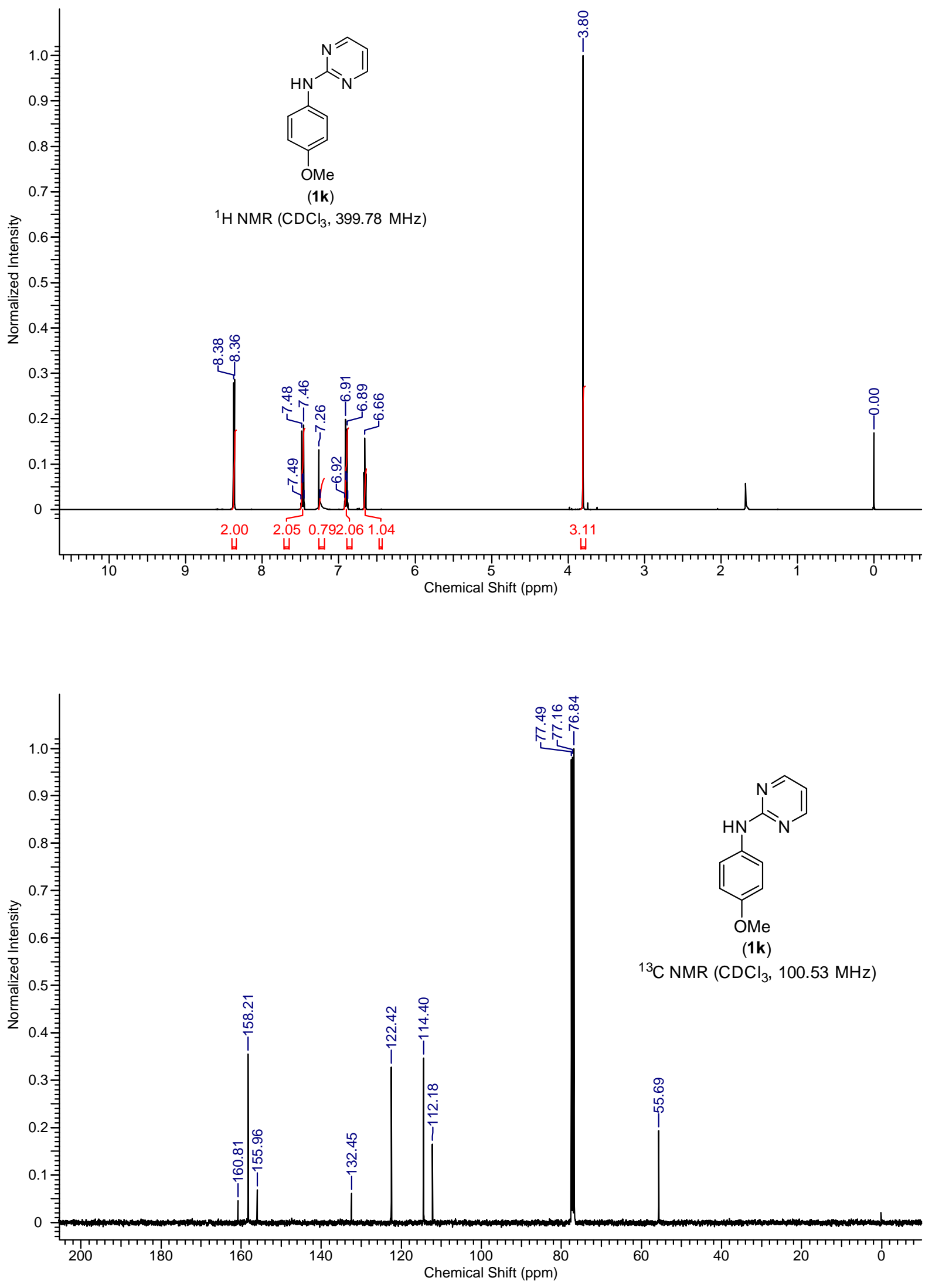

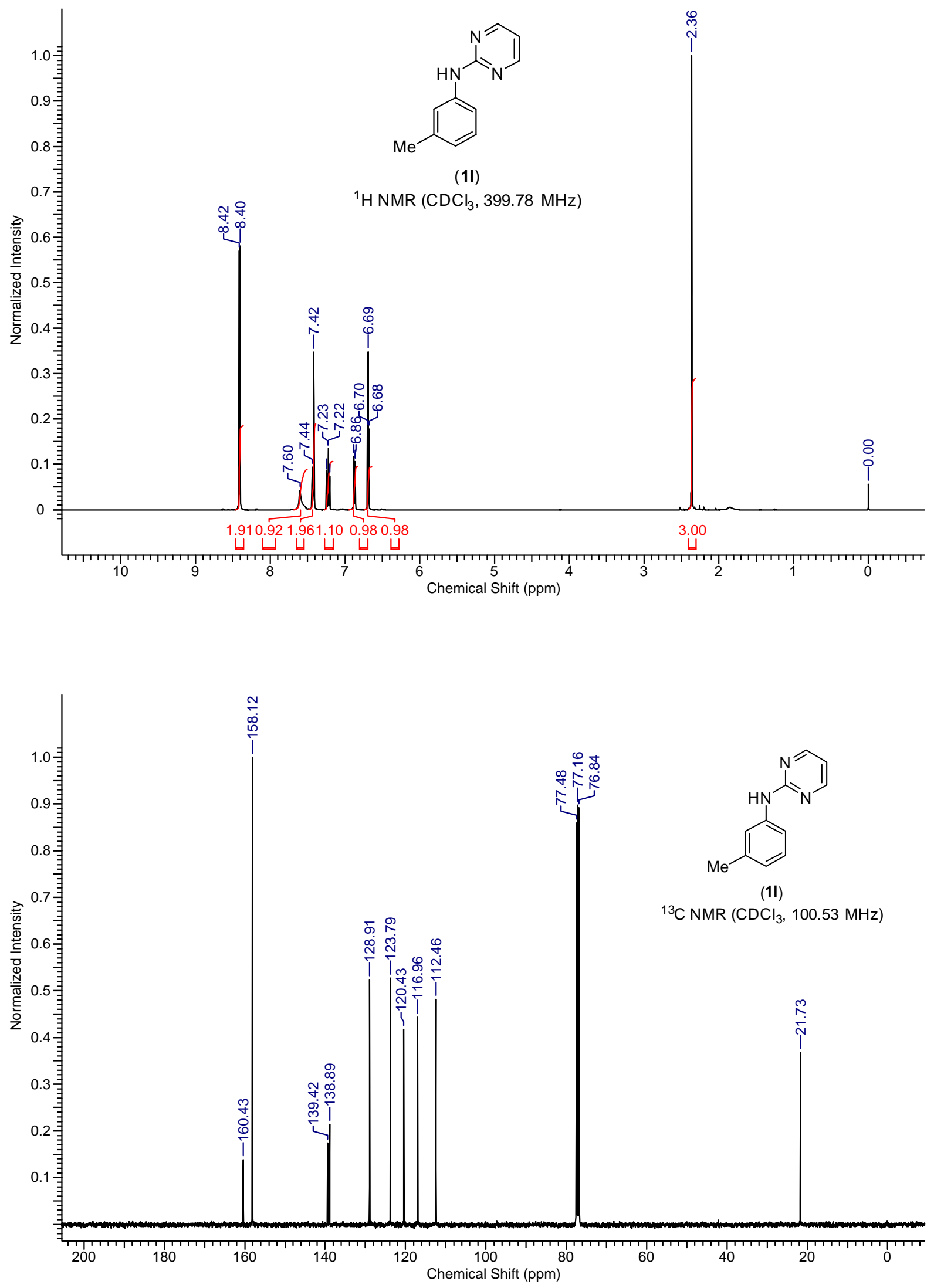

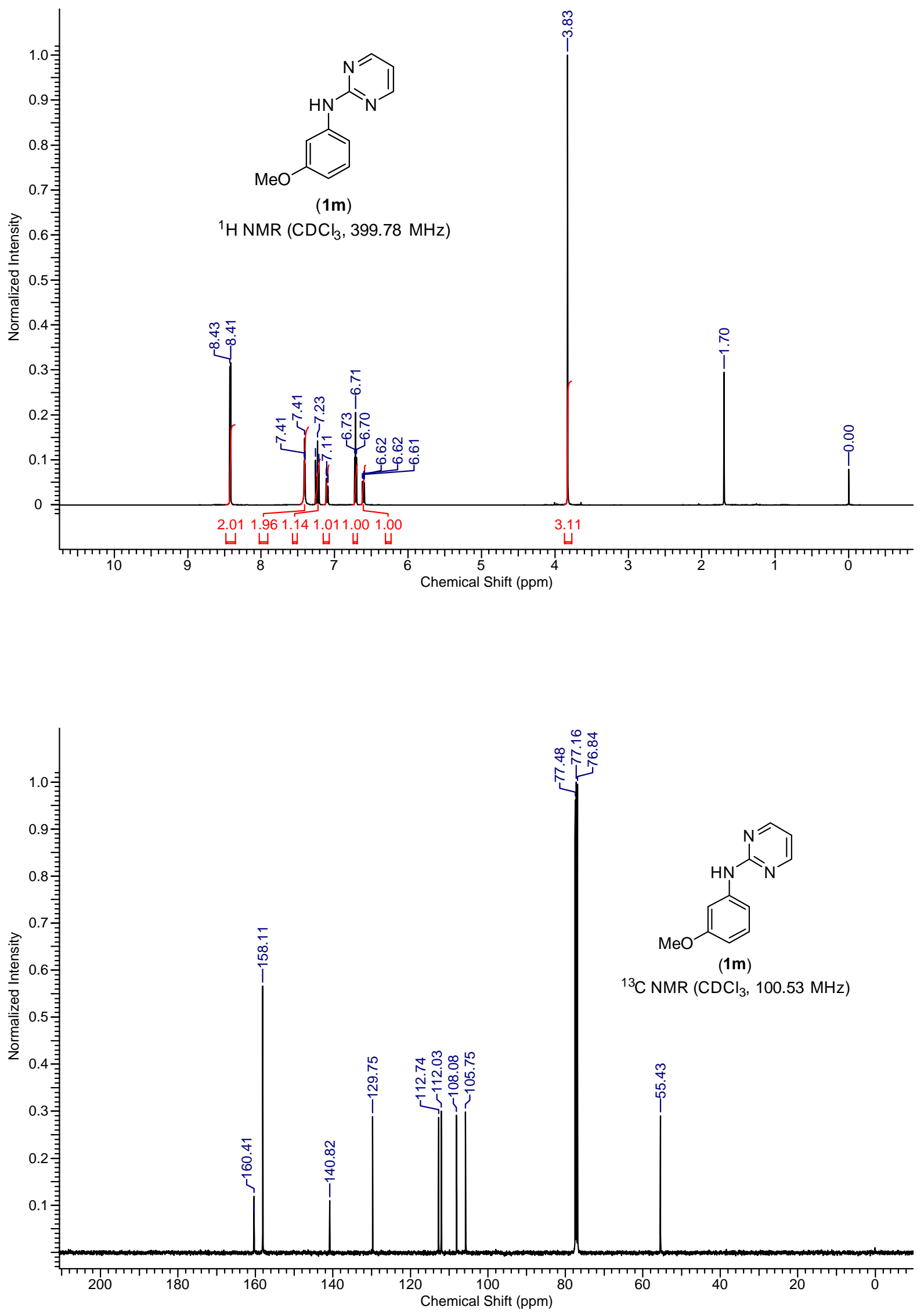

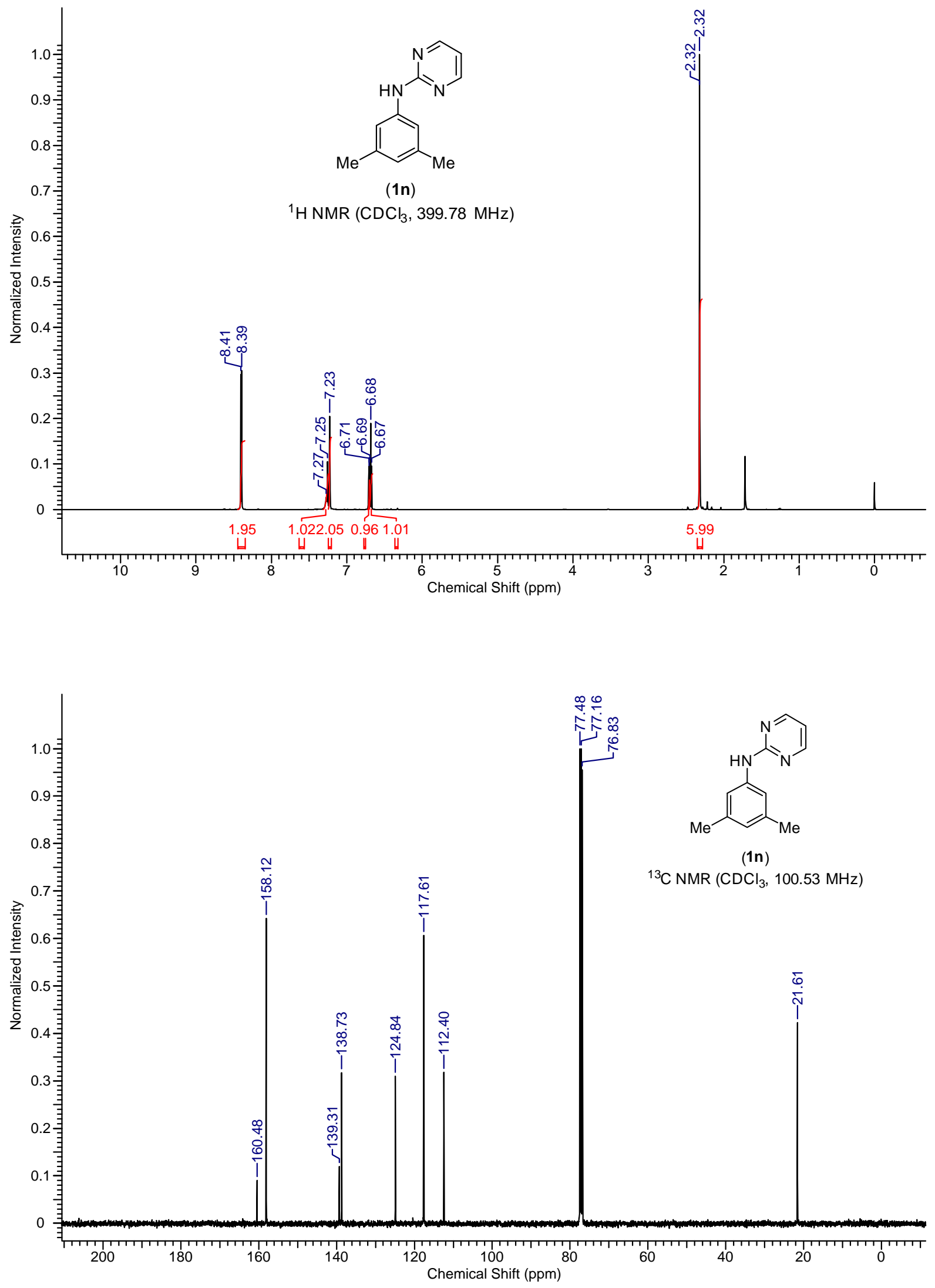


\section{2) Spectra of Compound 3}
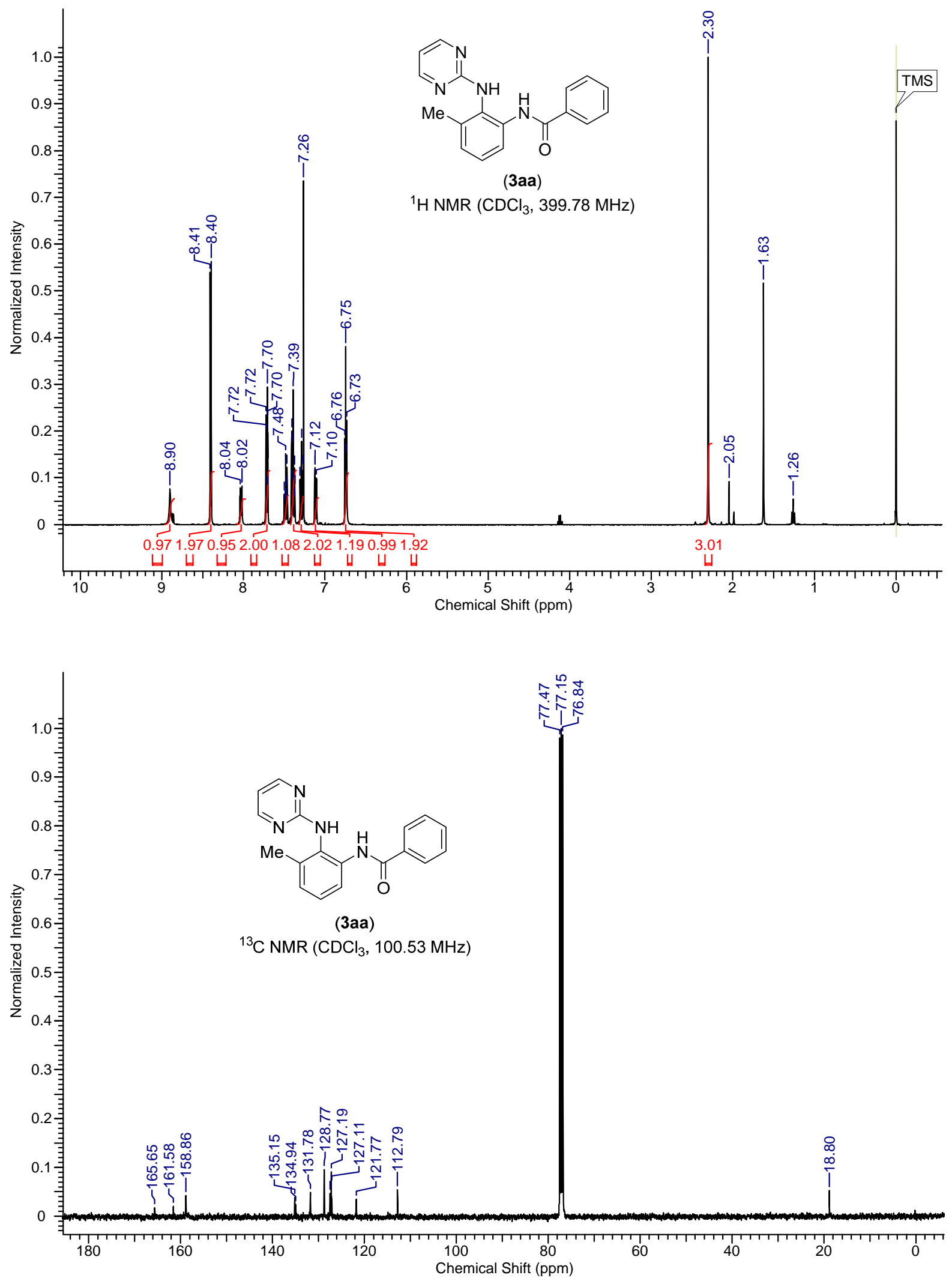

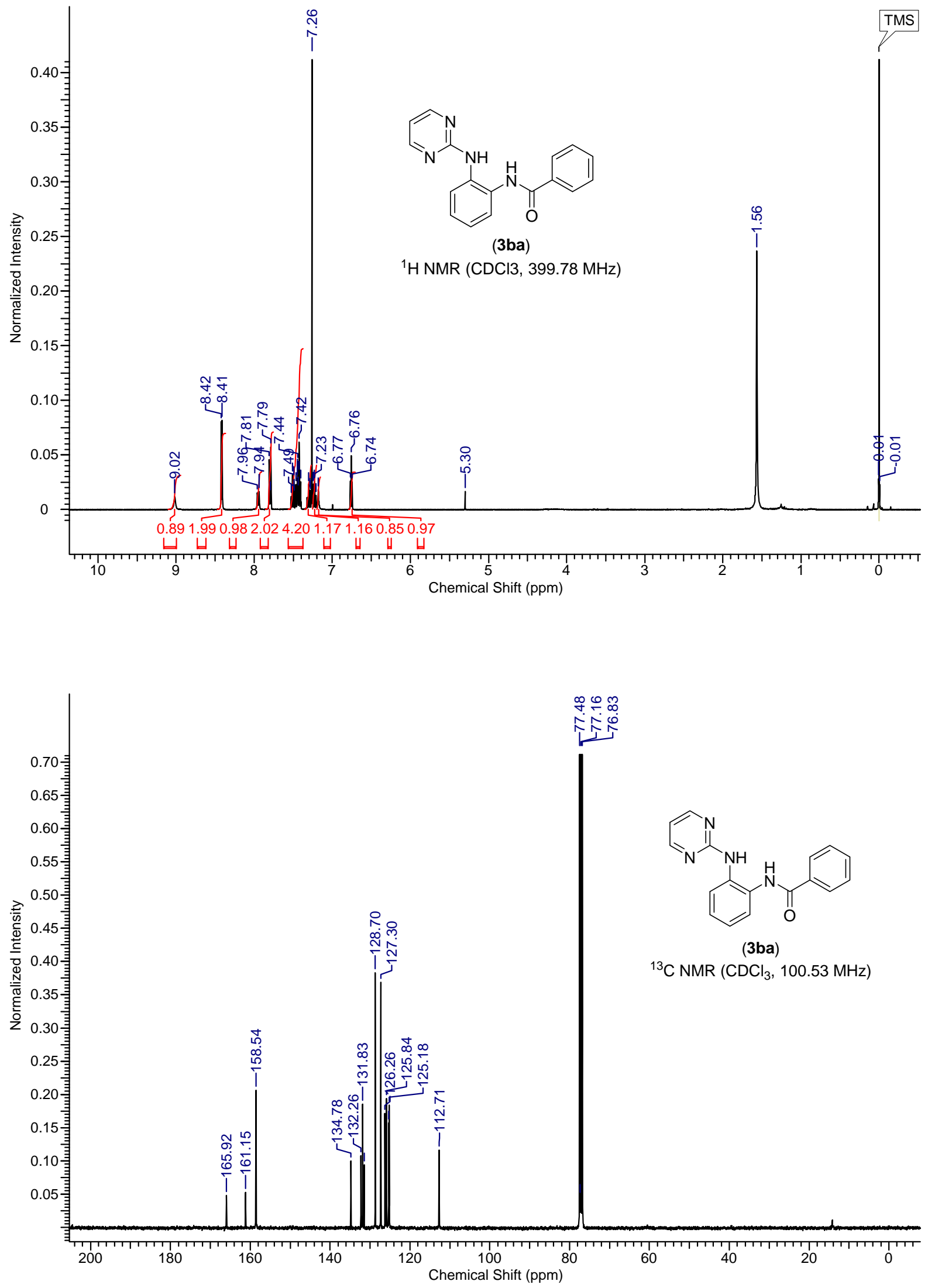

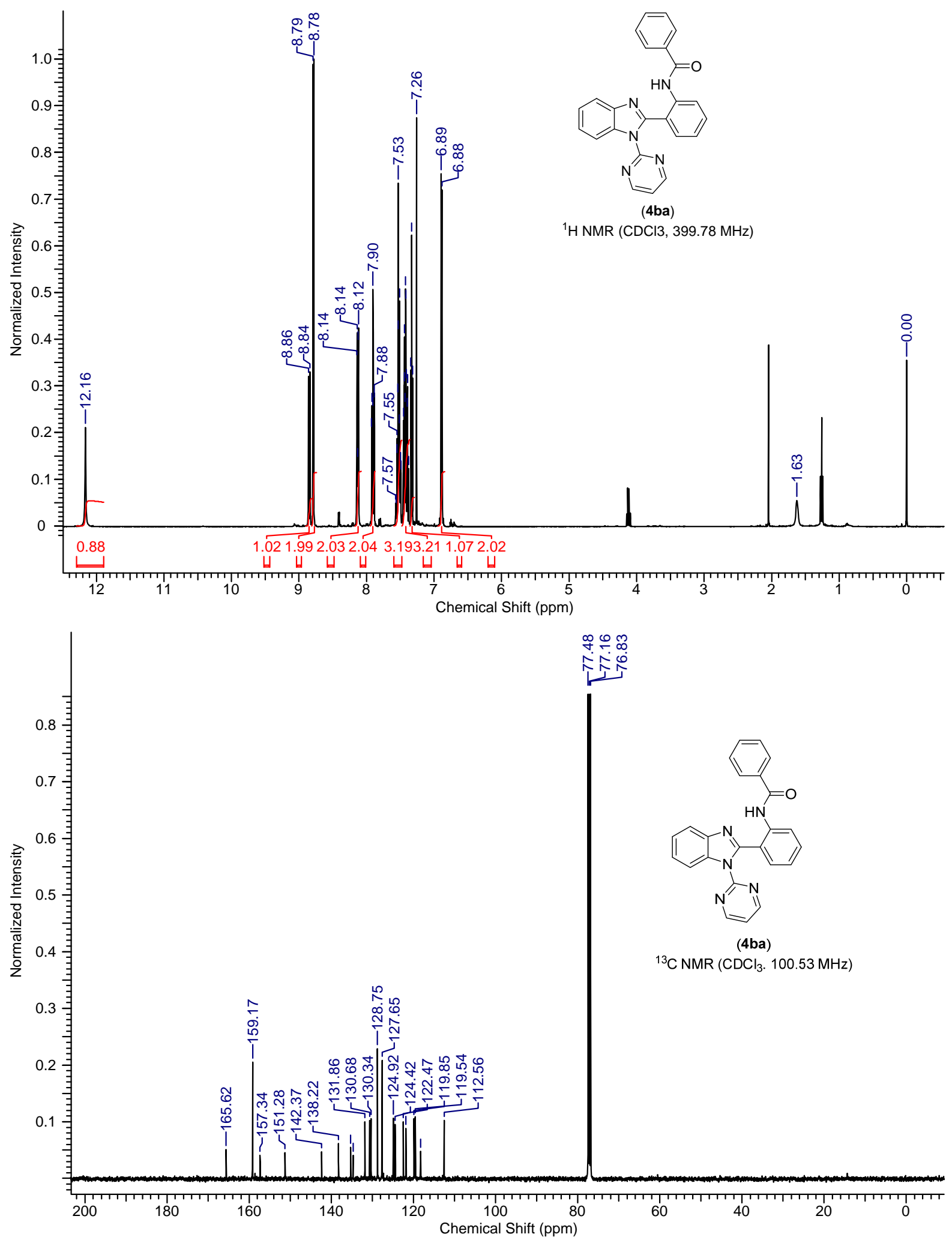

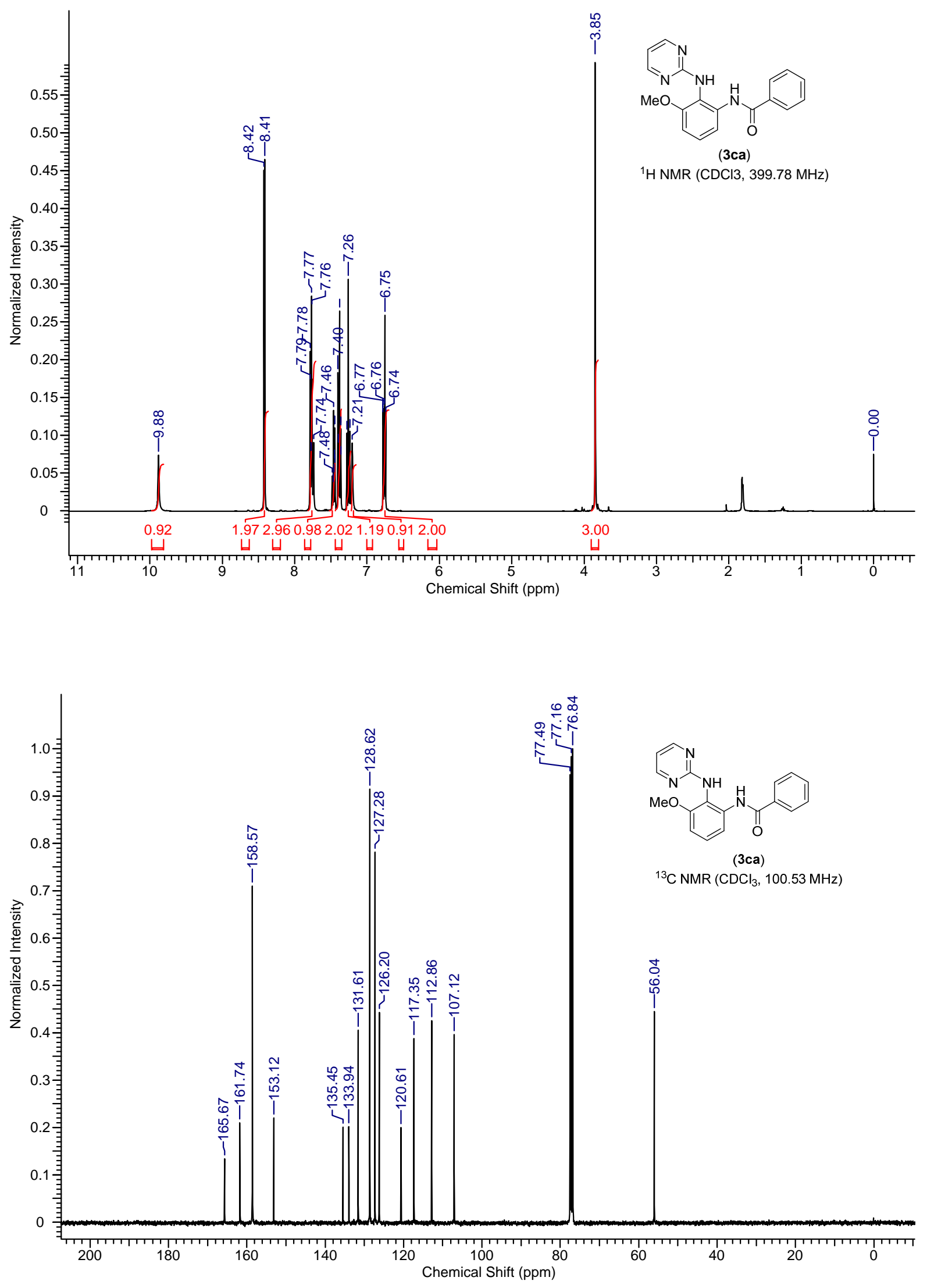

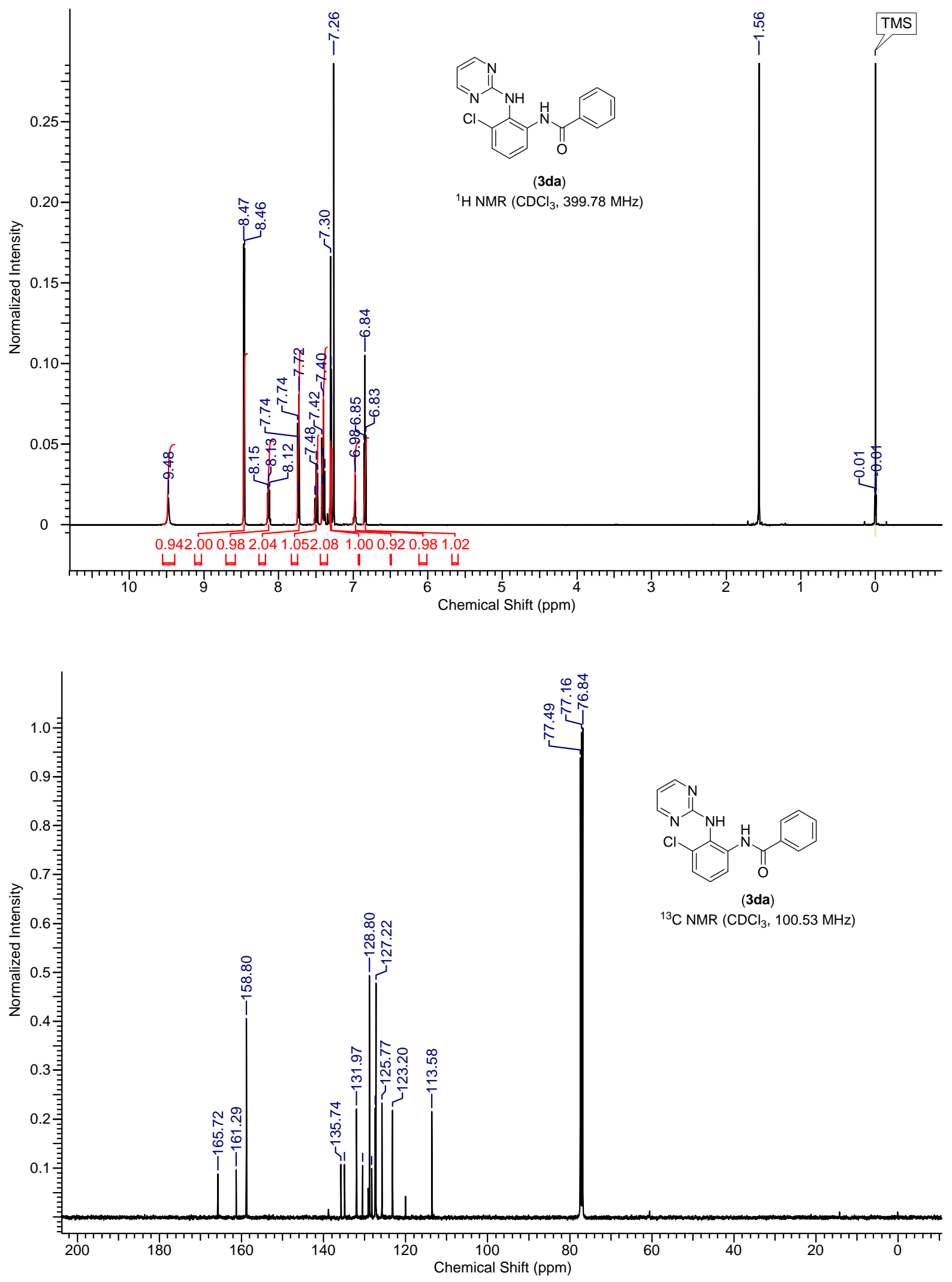

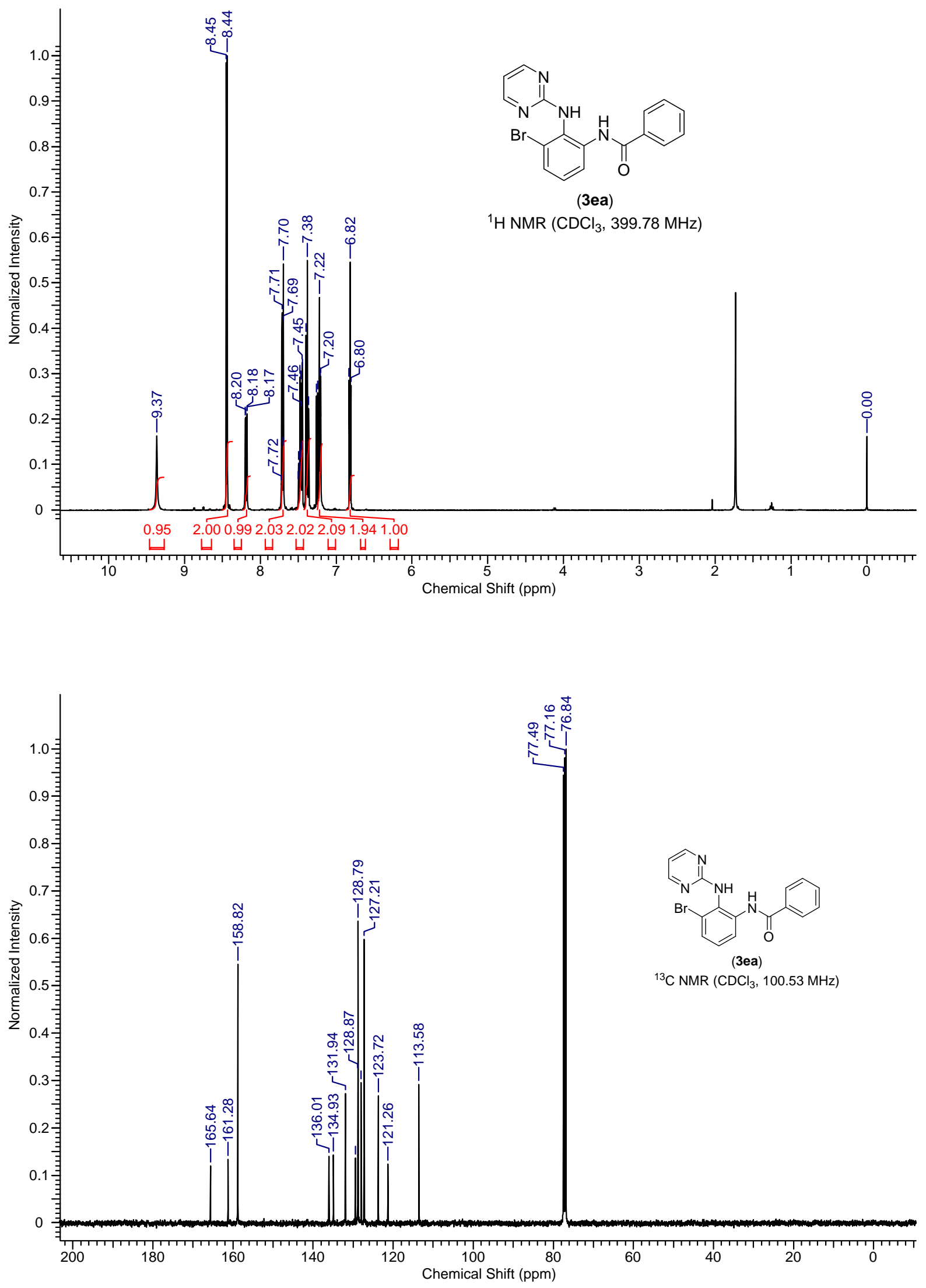

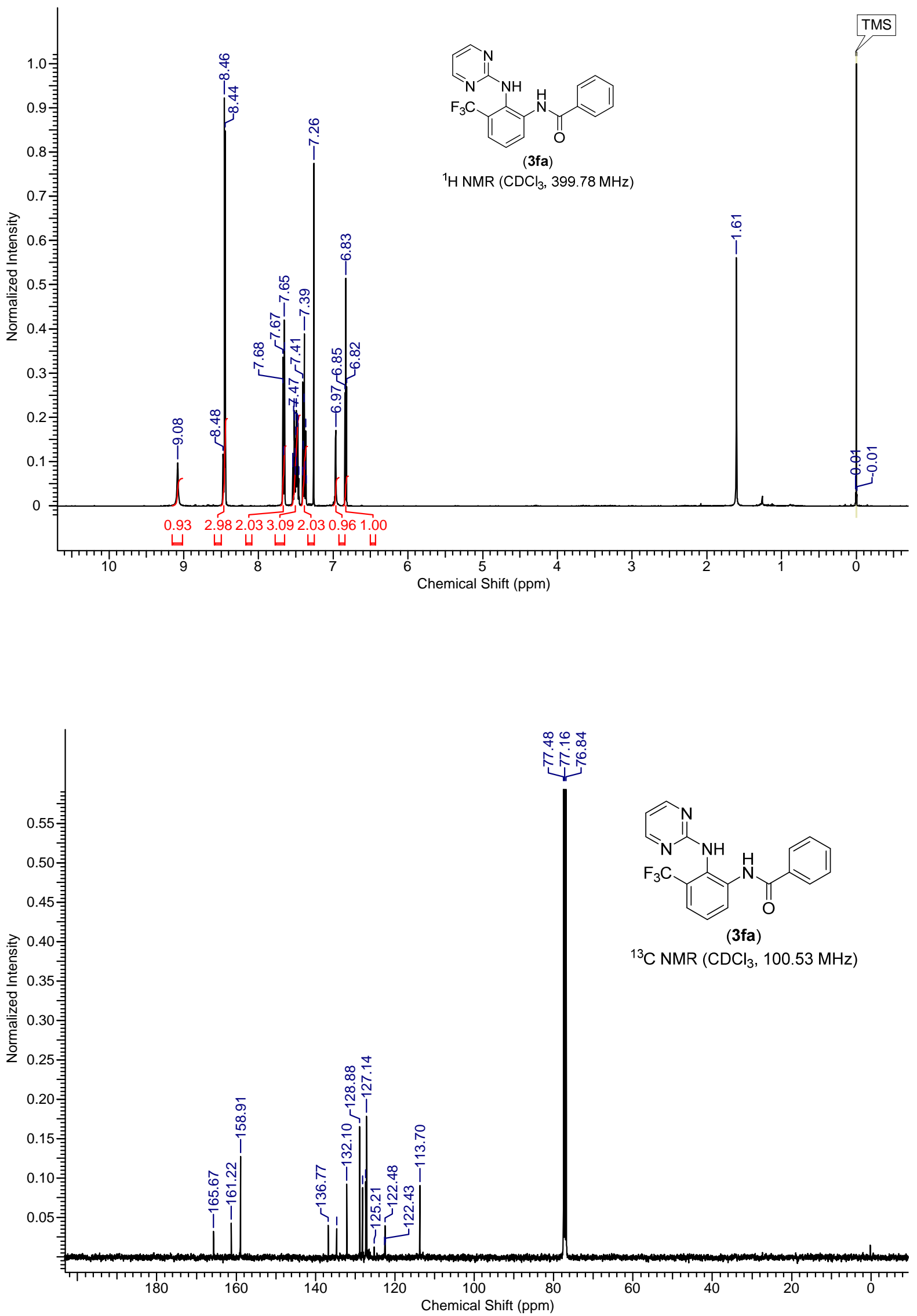

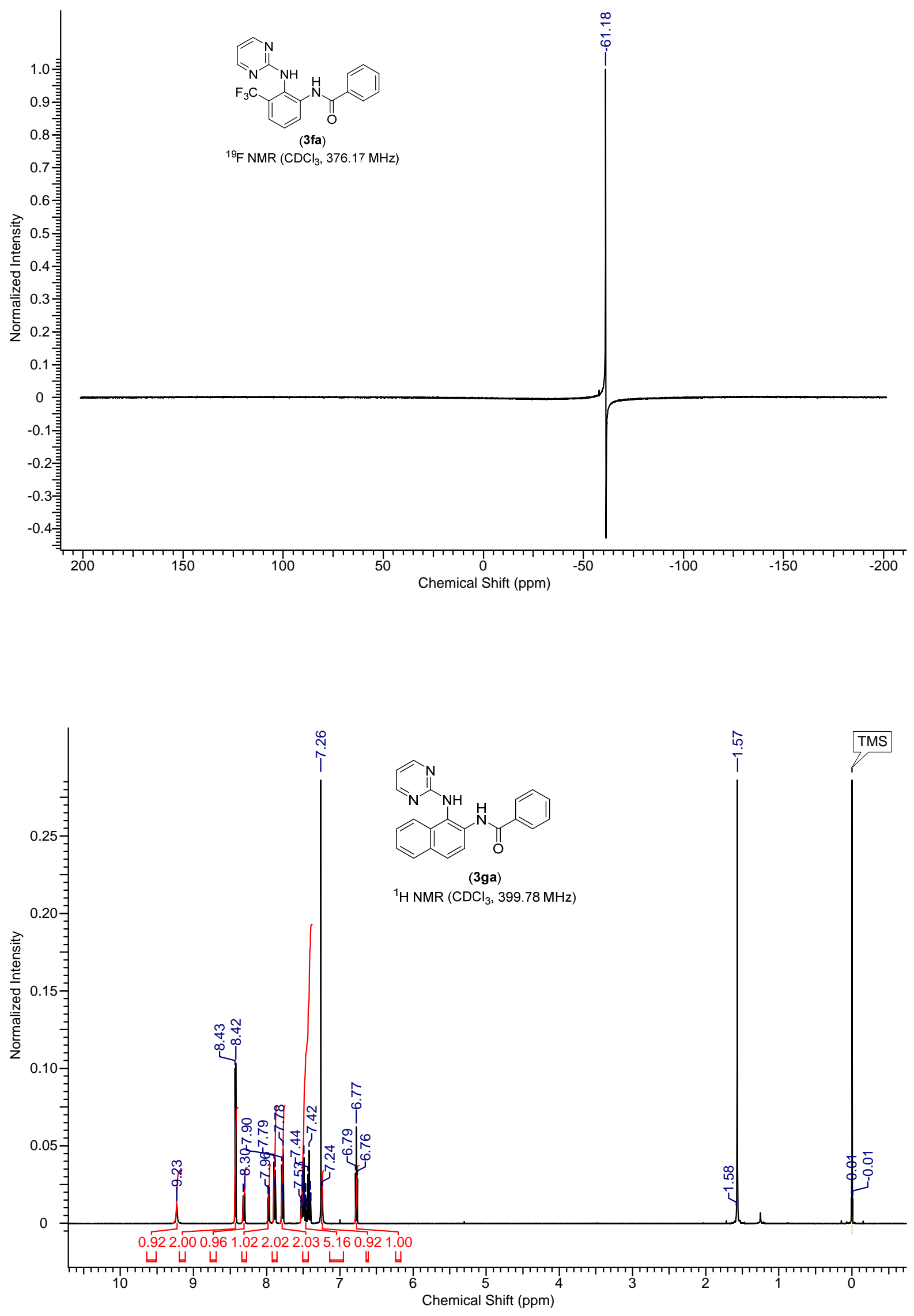

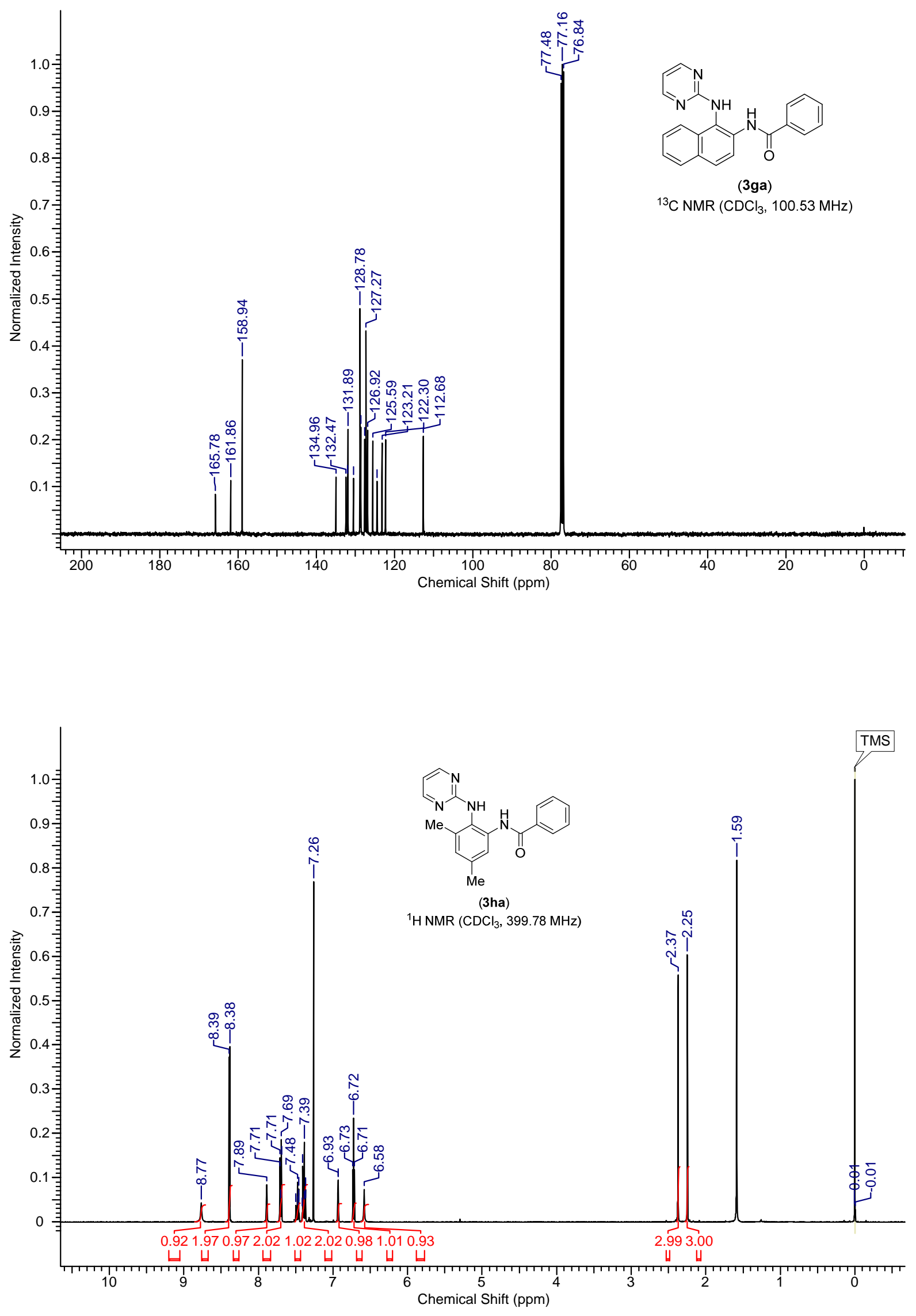

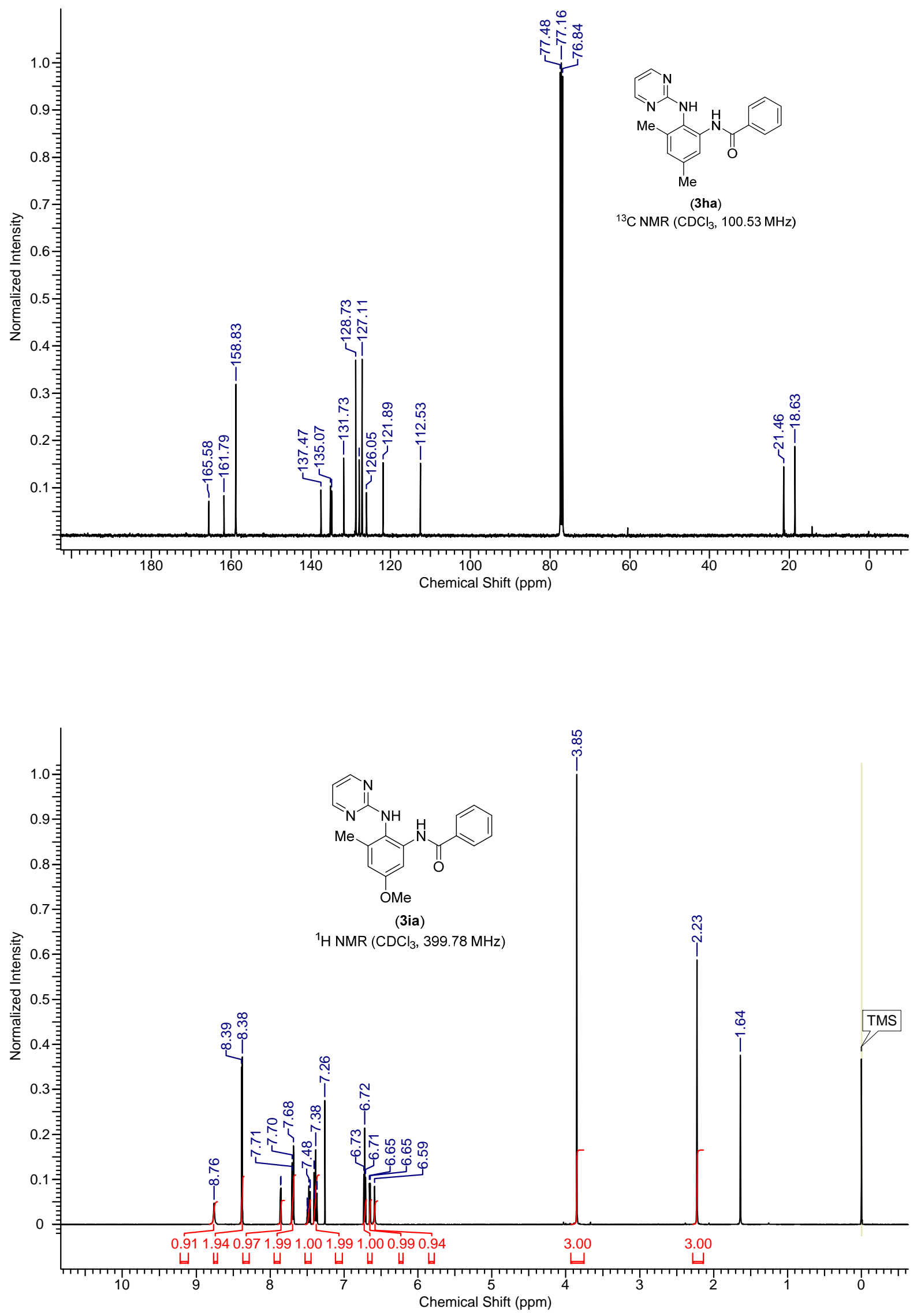

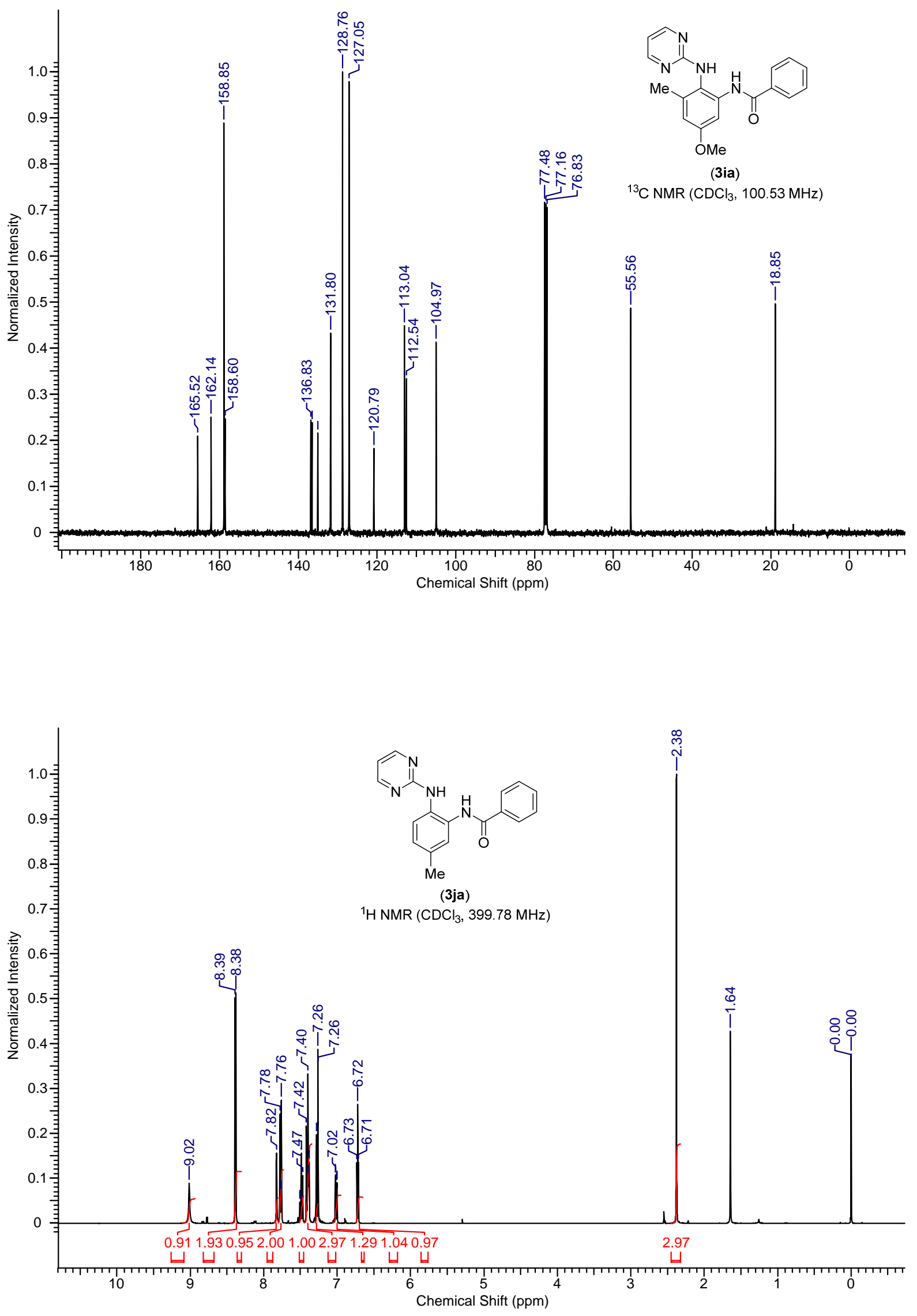

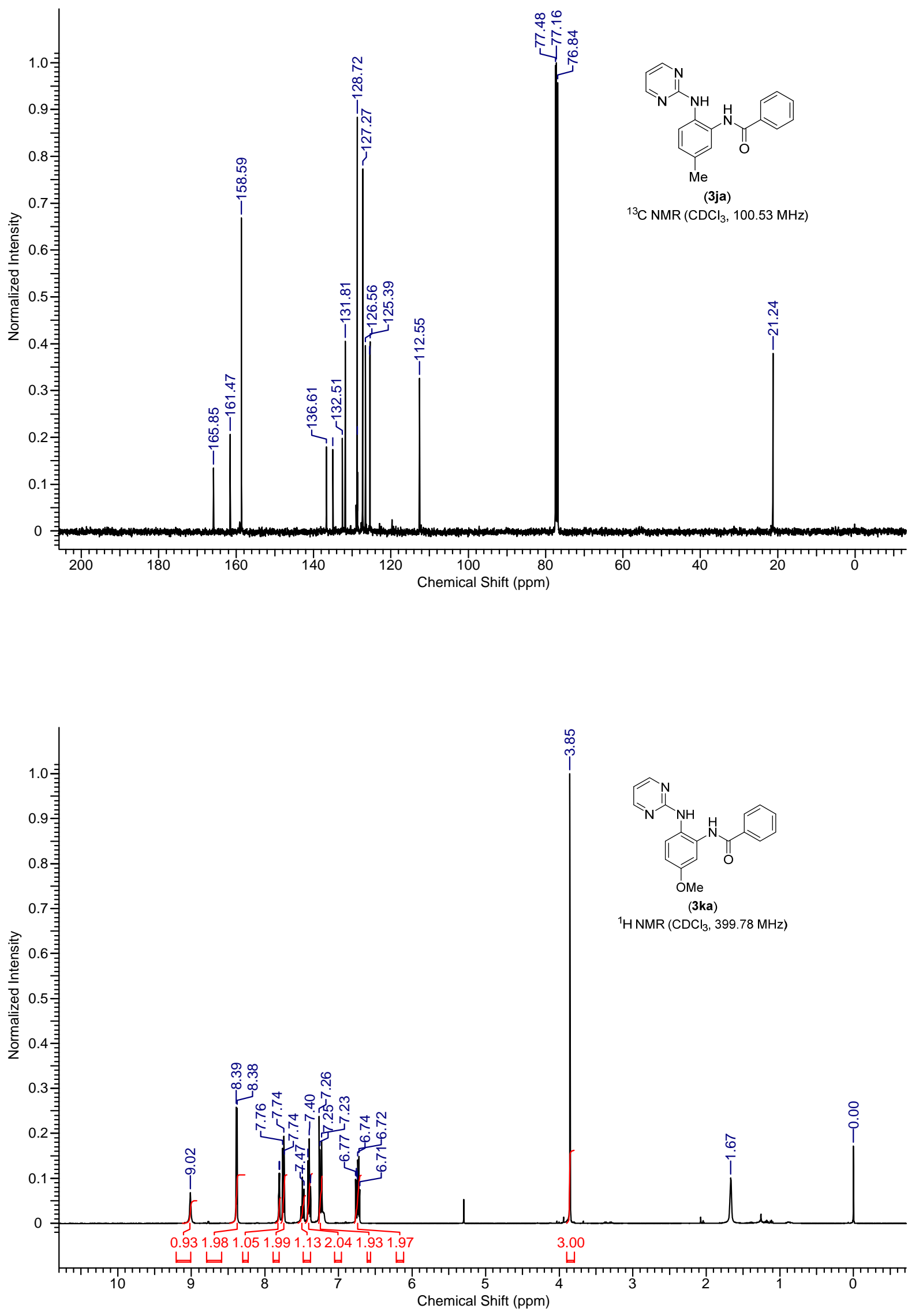

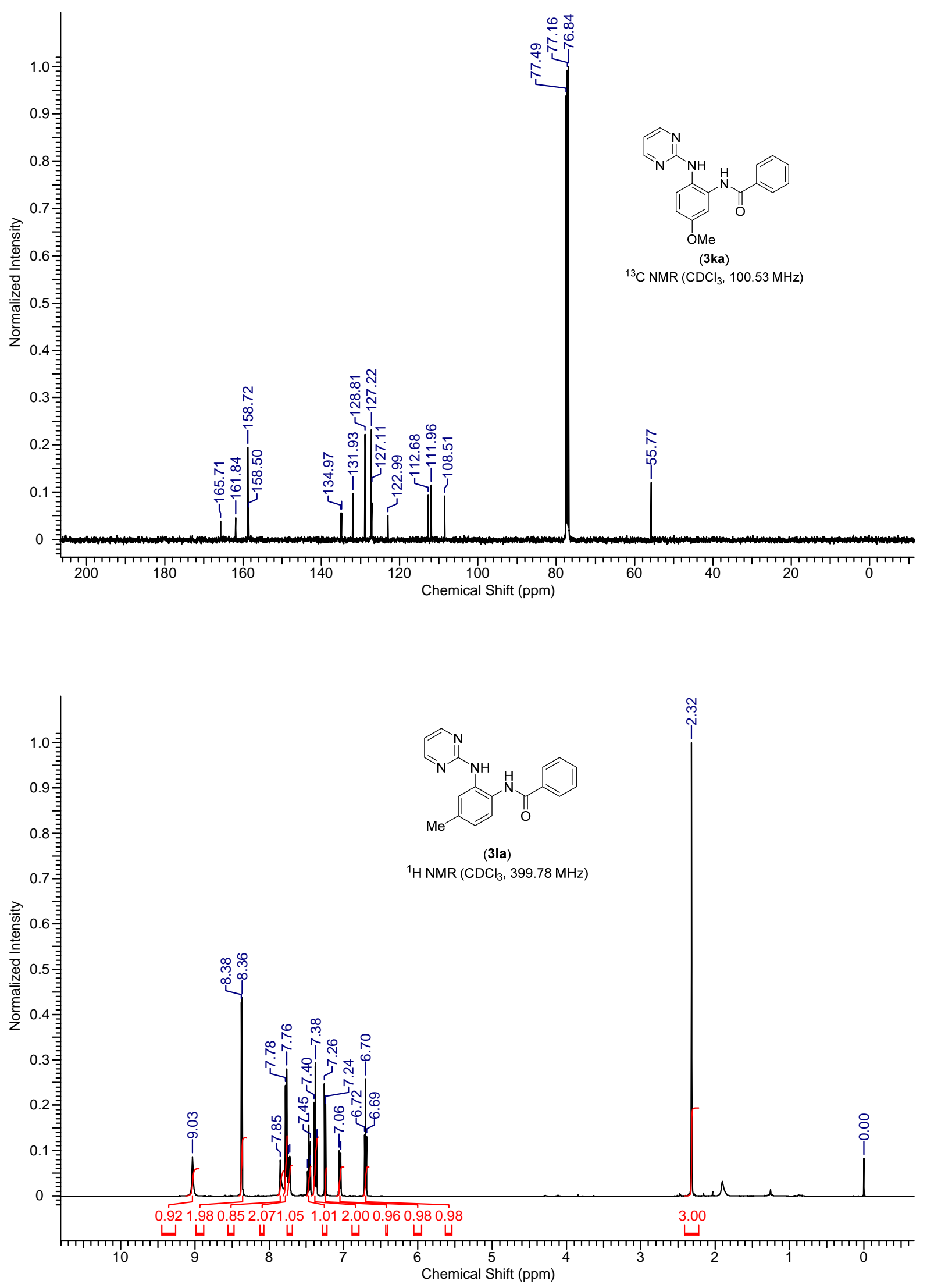

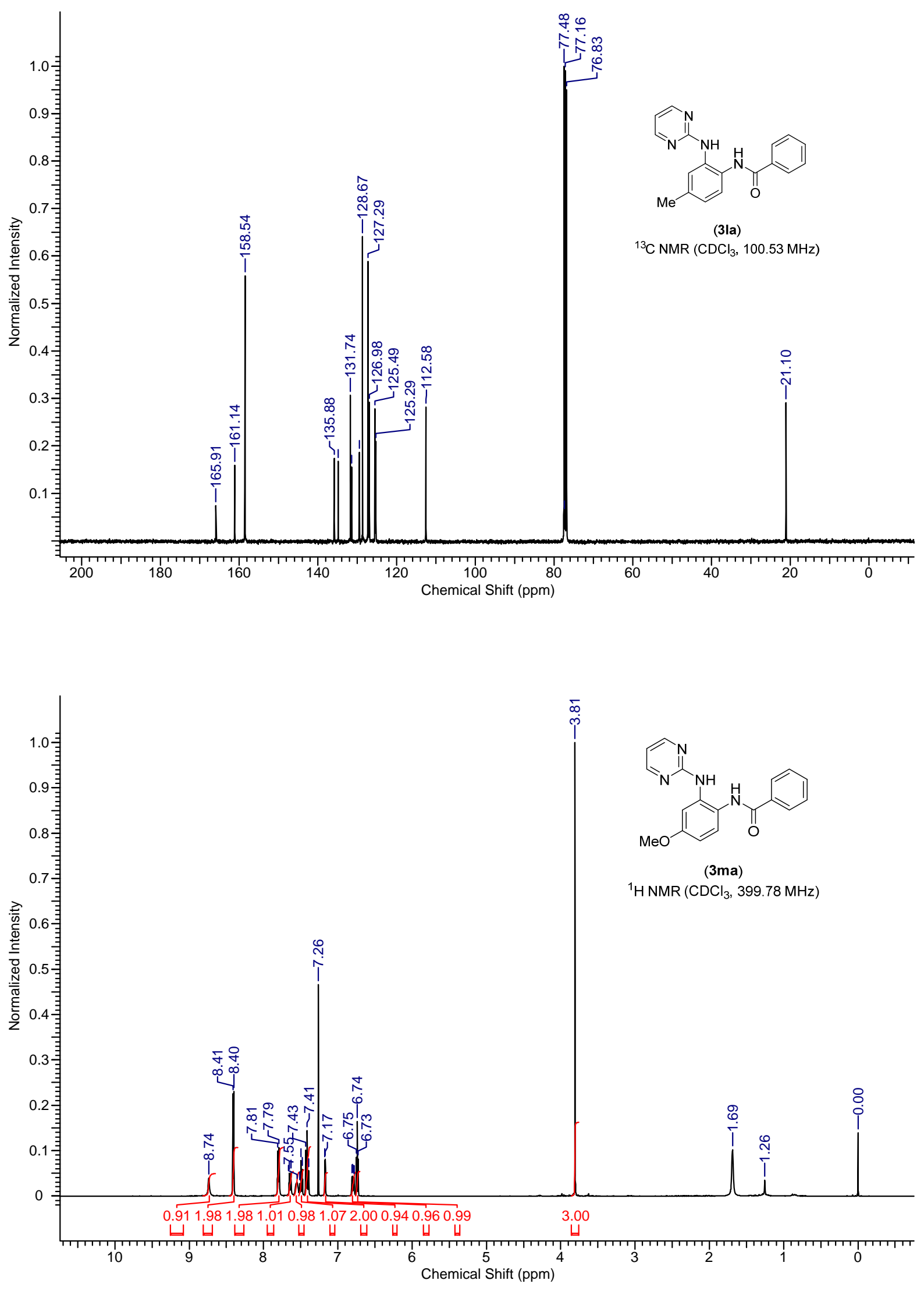

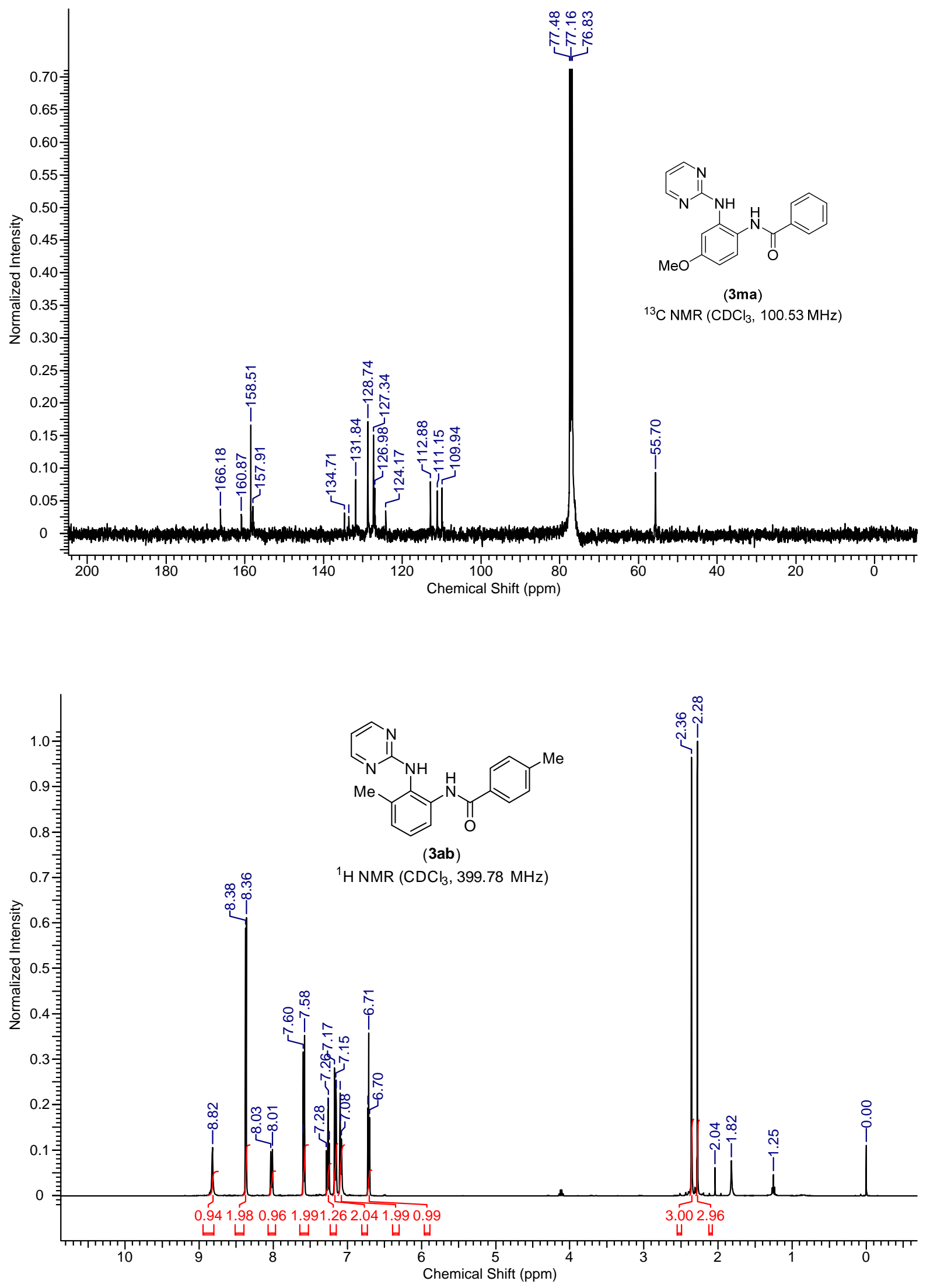

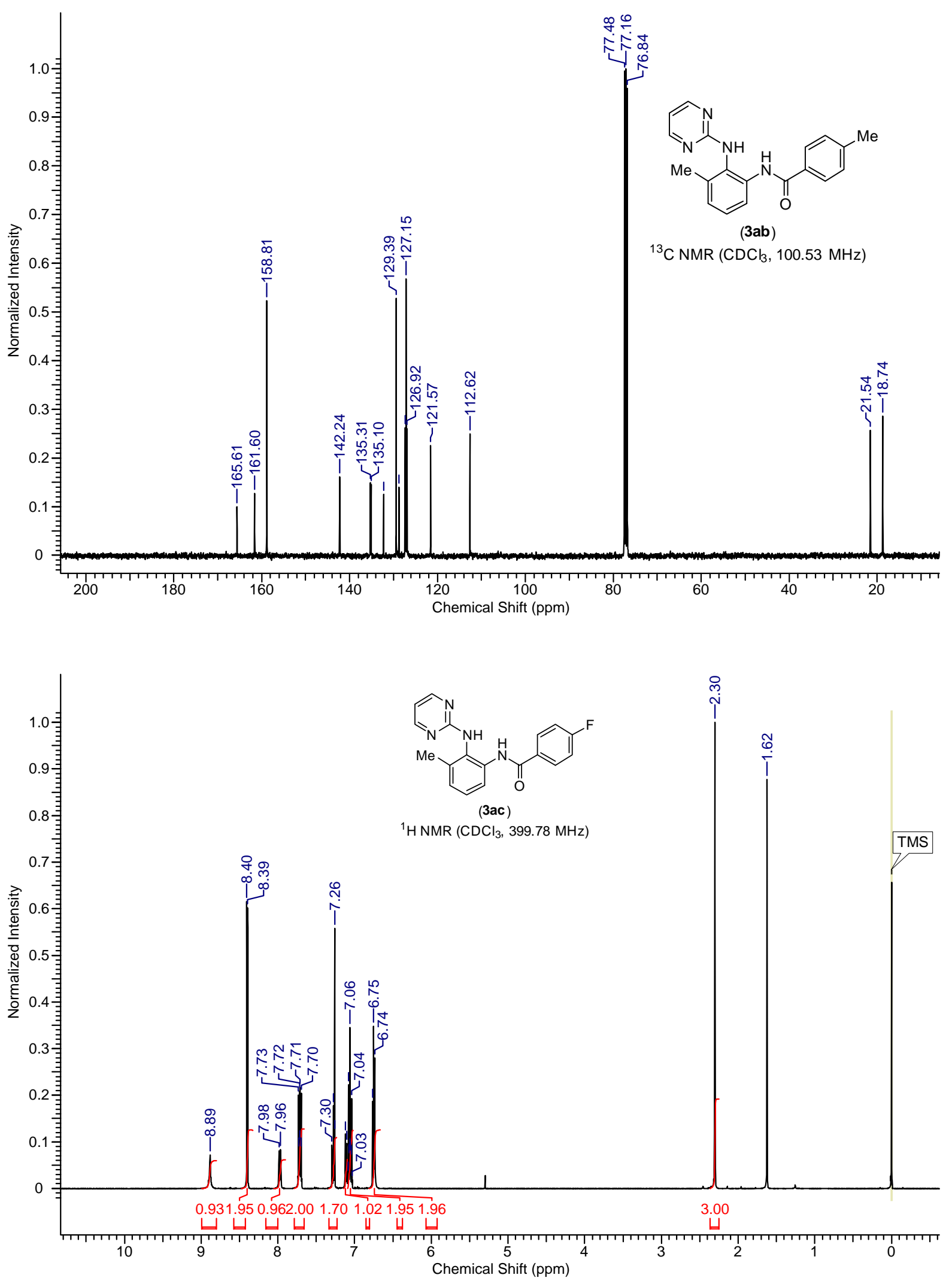

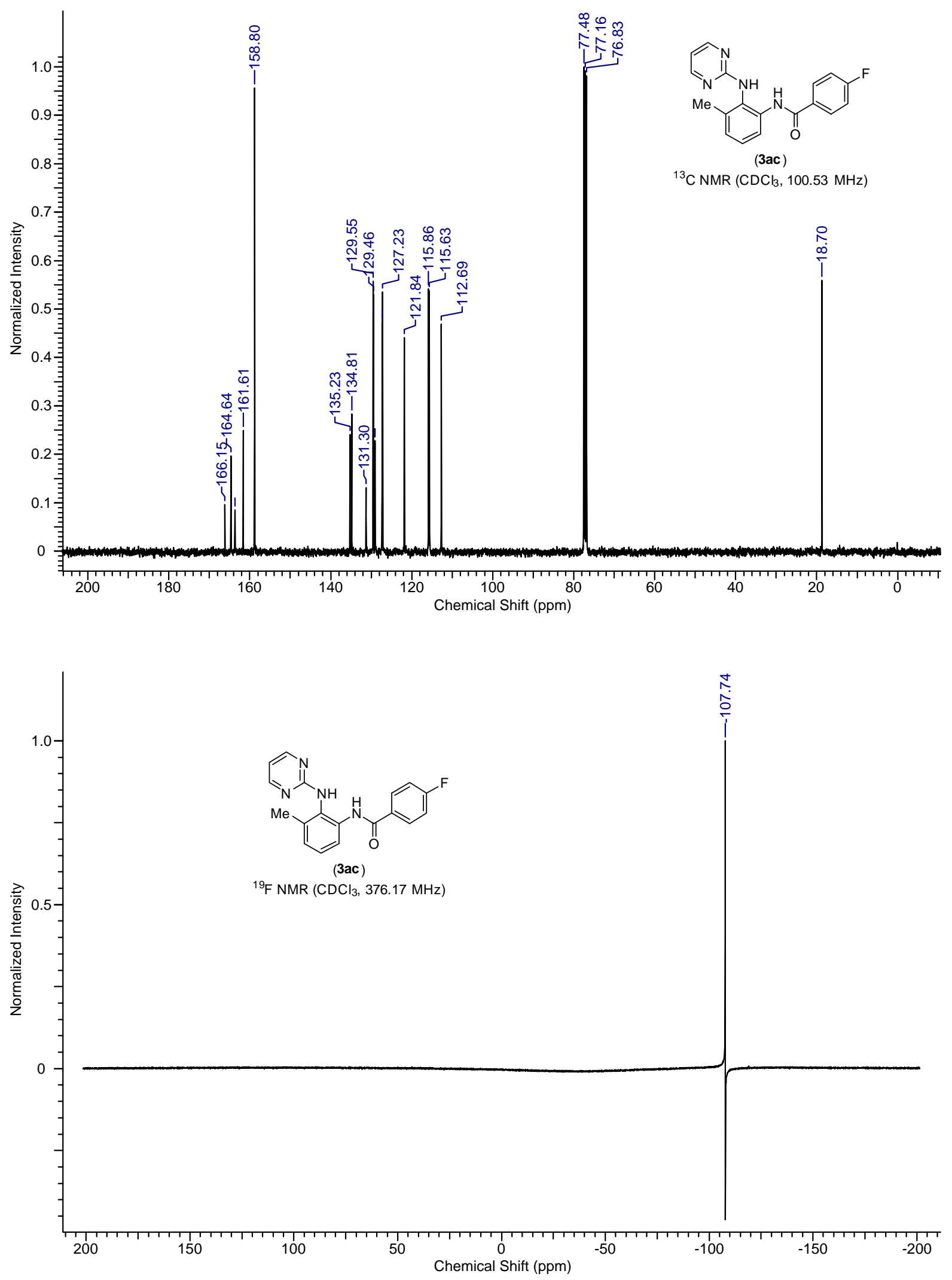

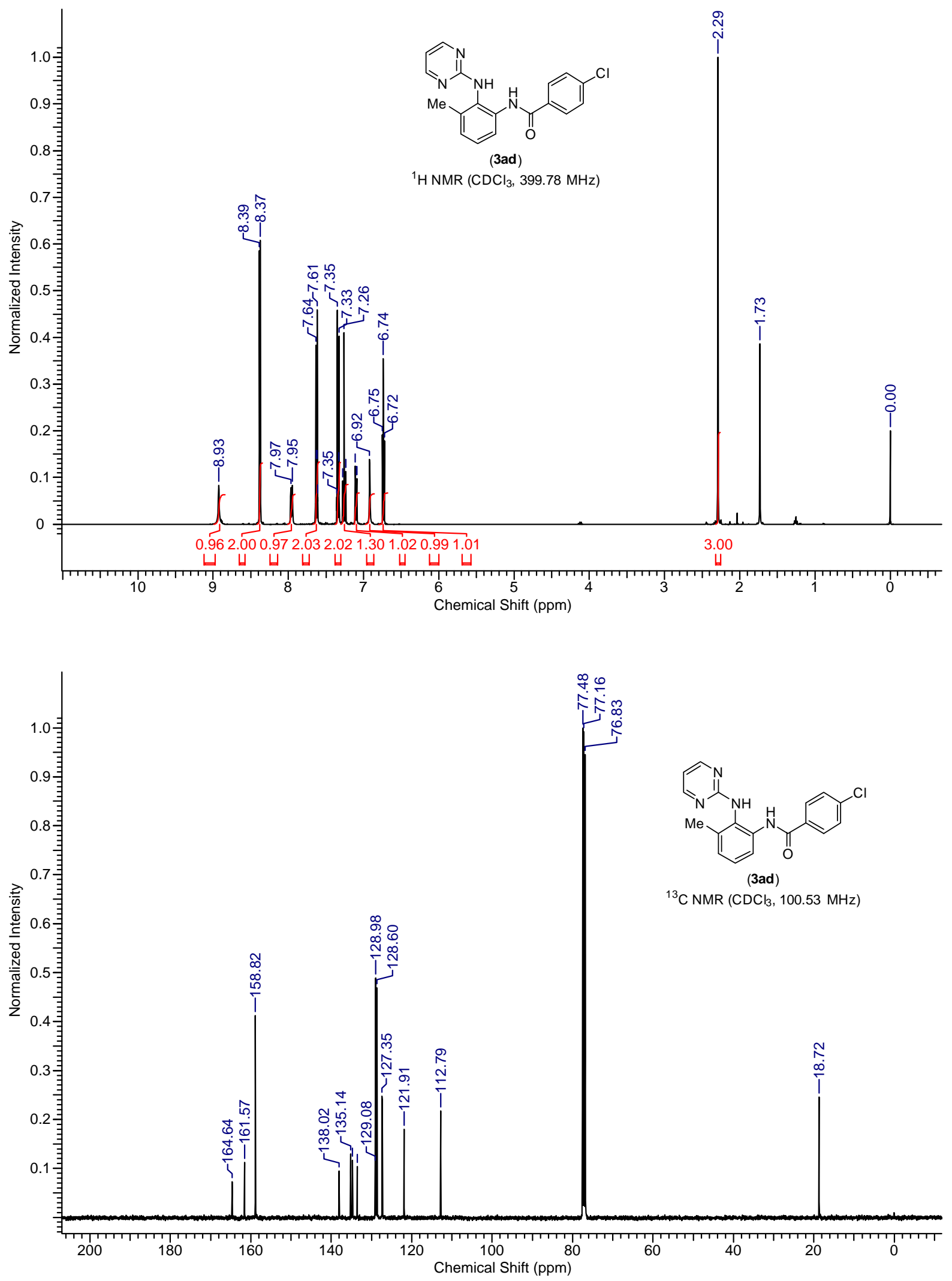

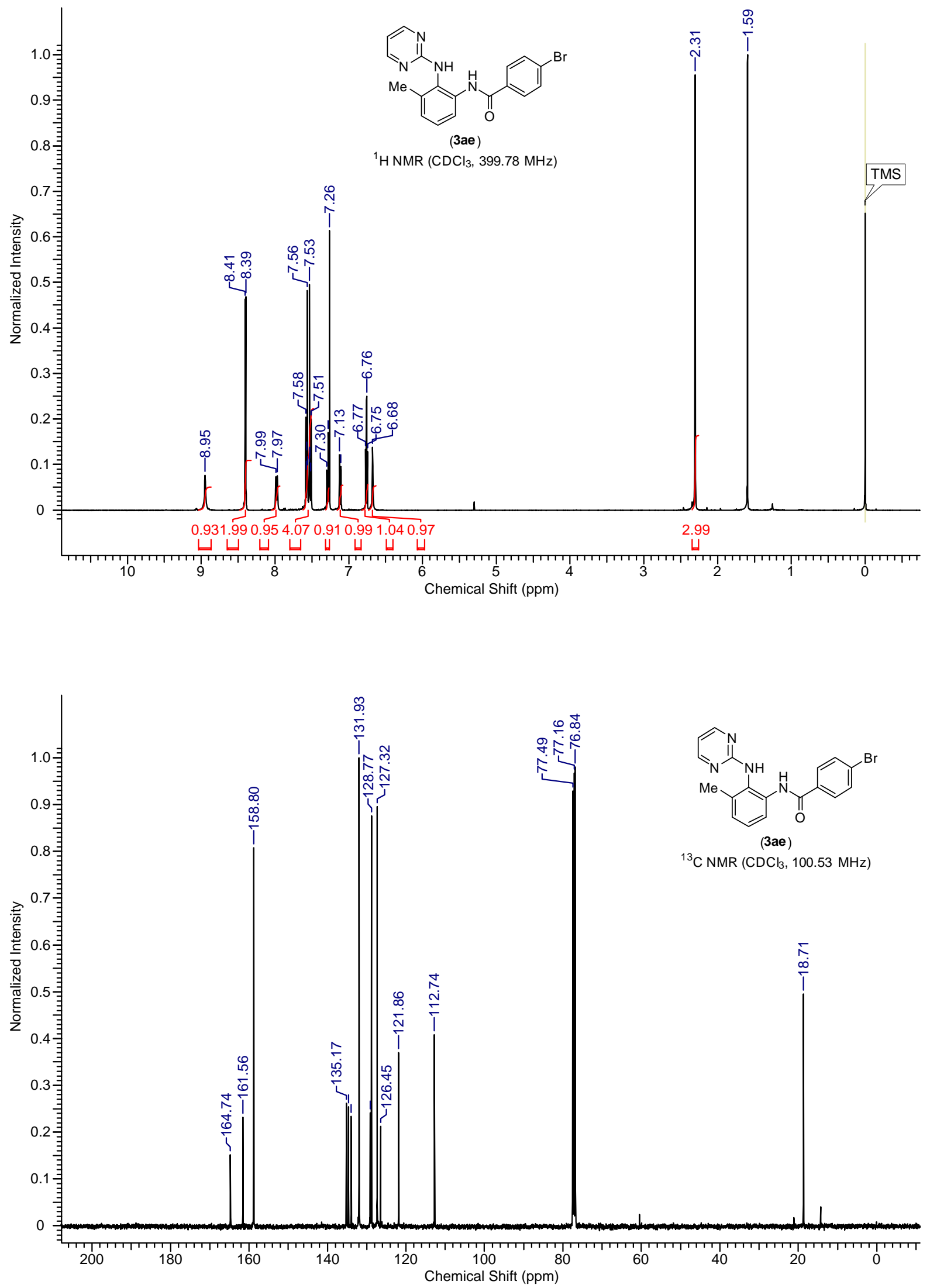

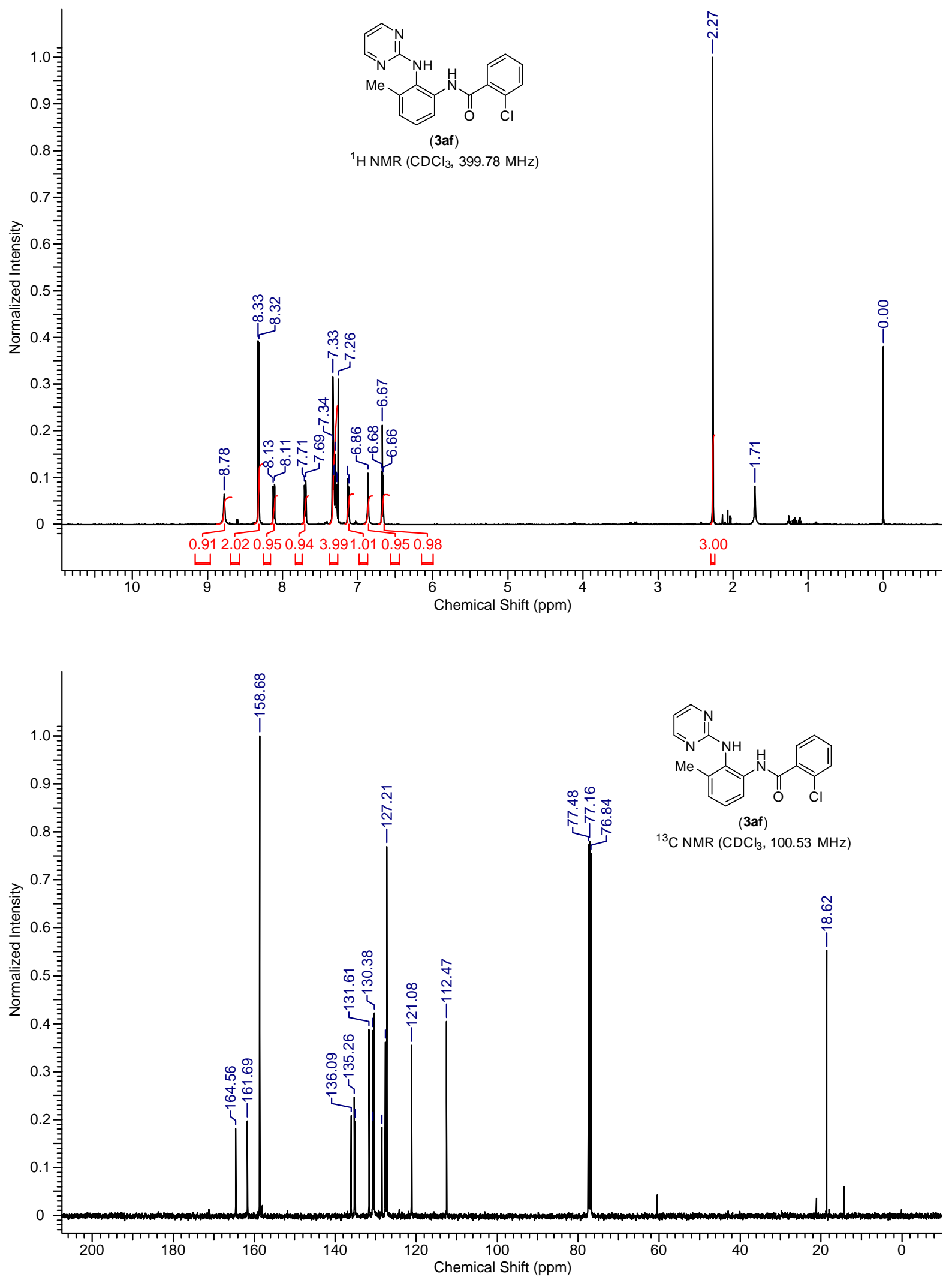

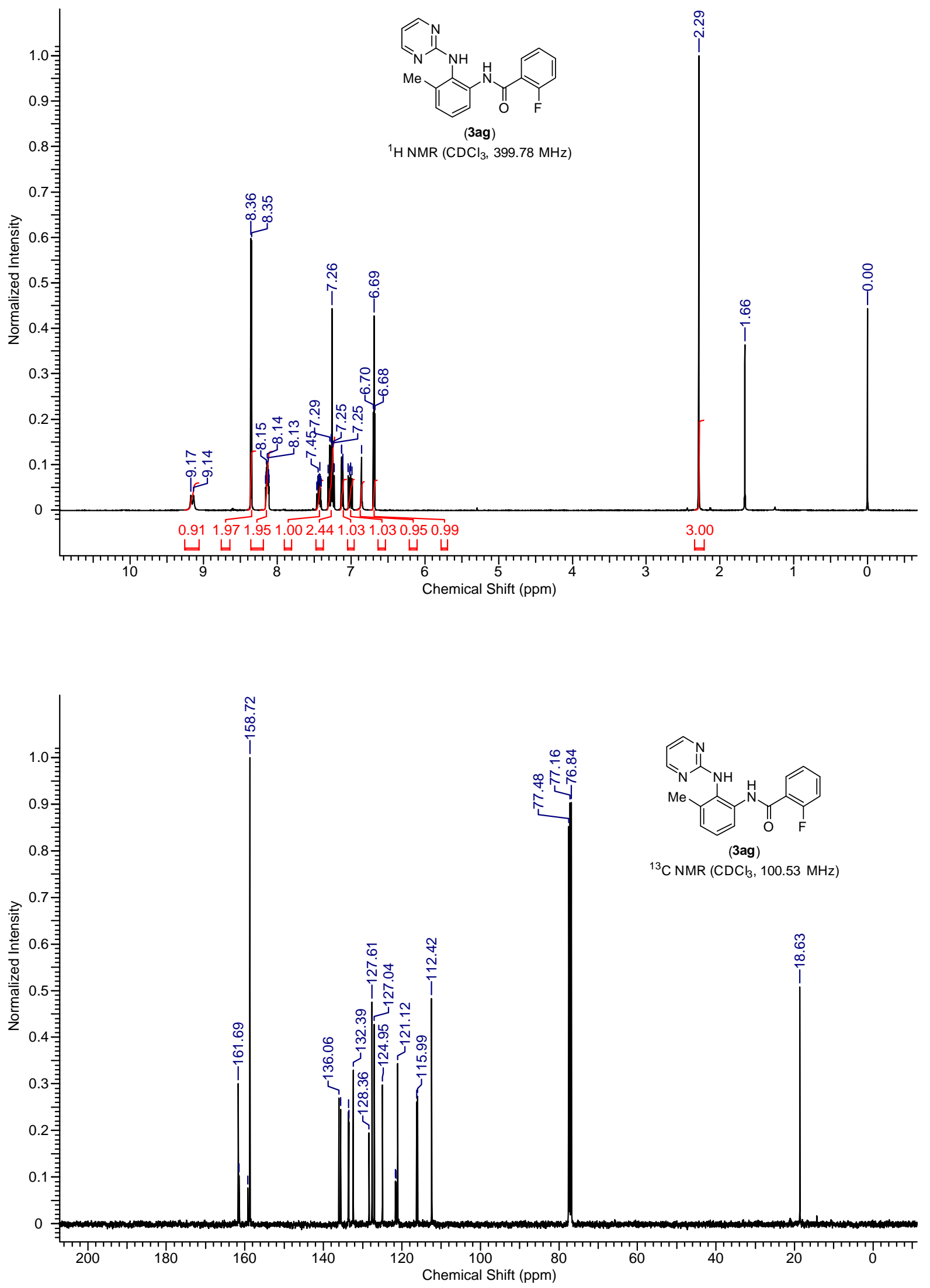

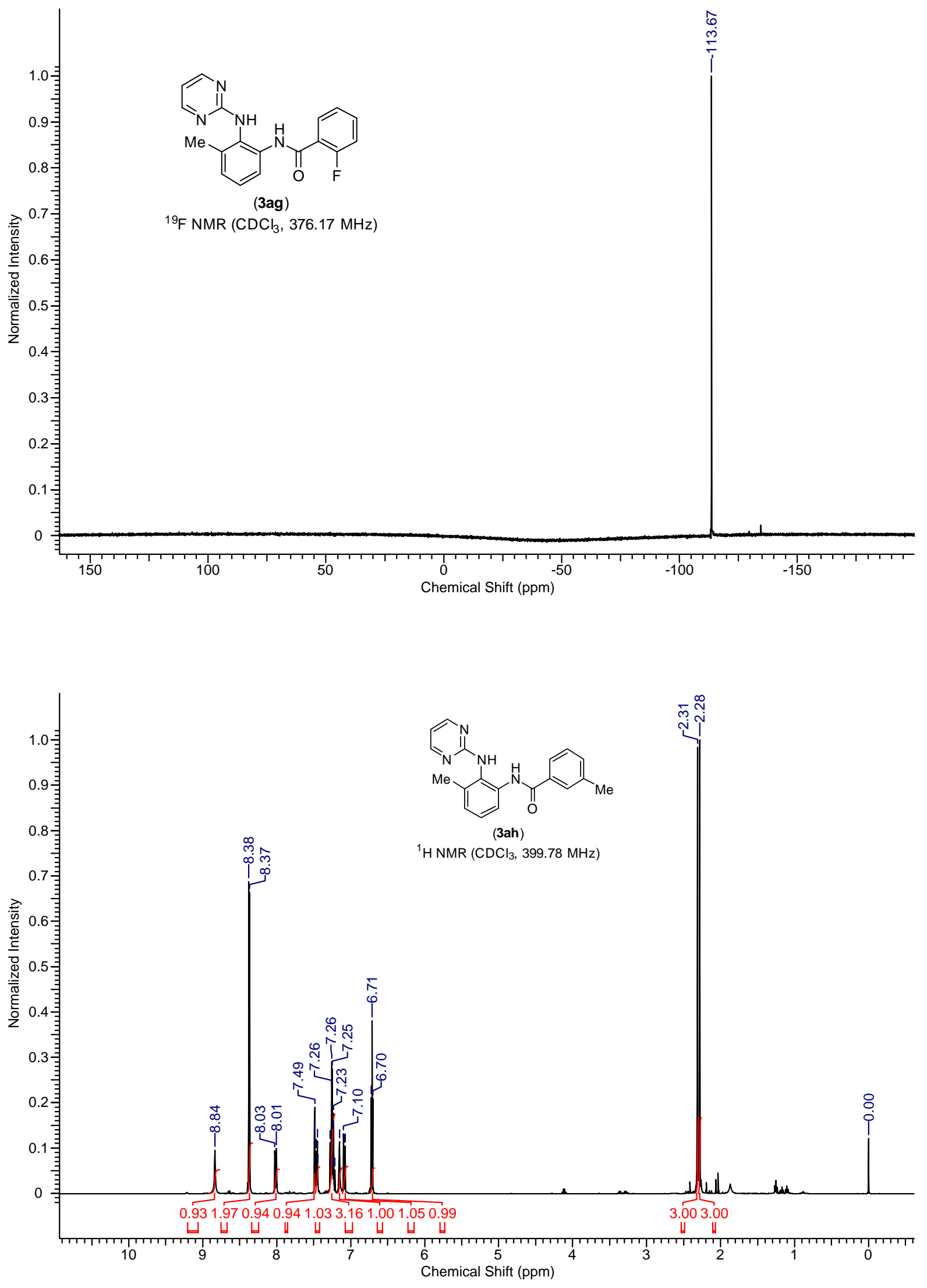

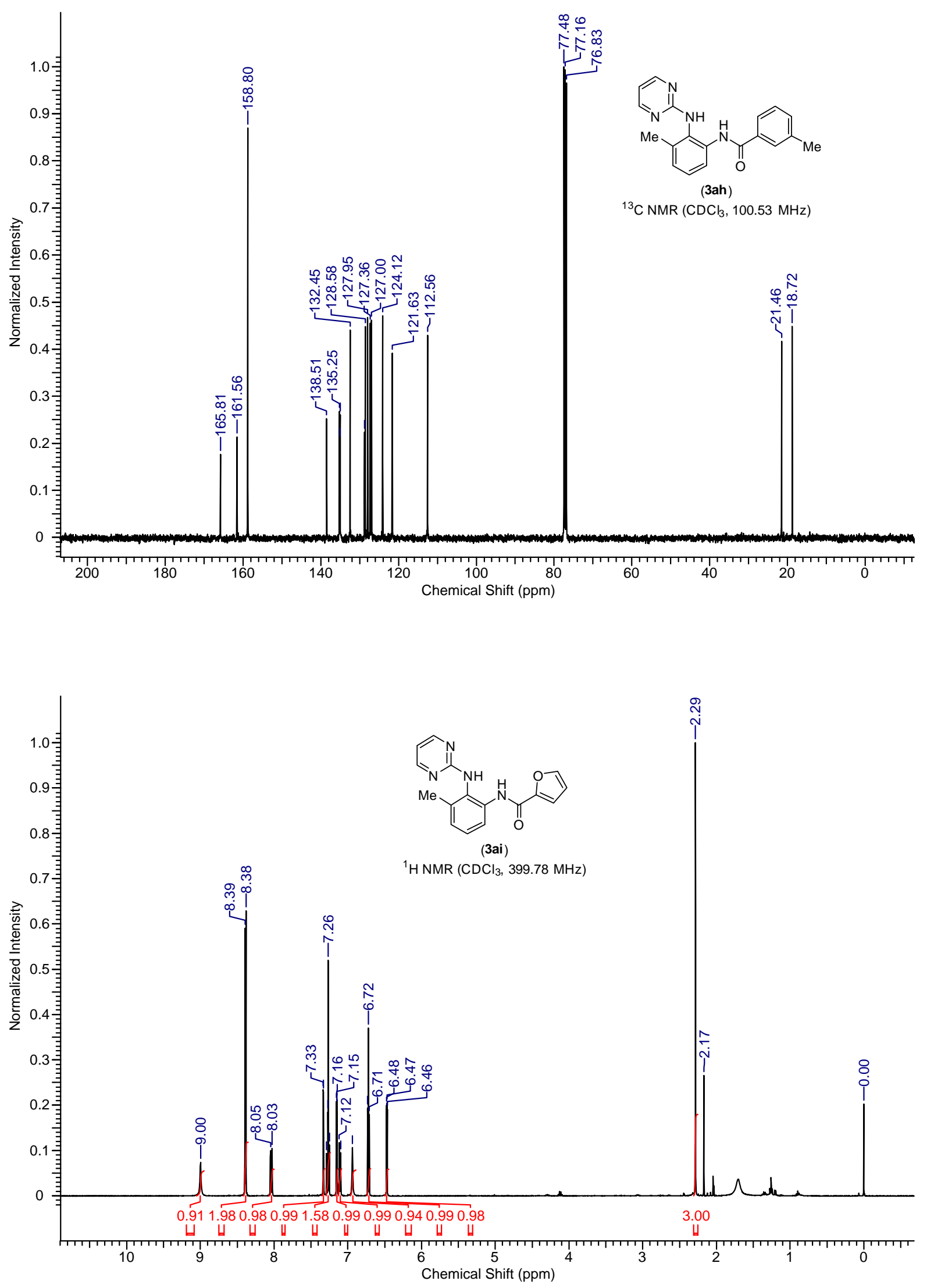

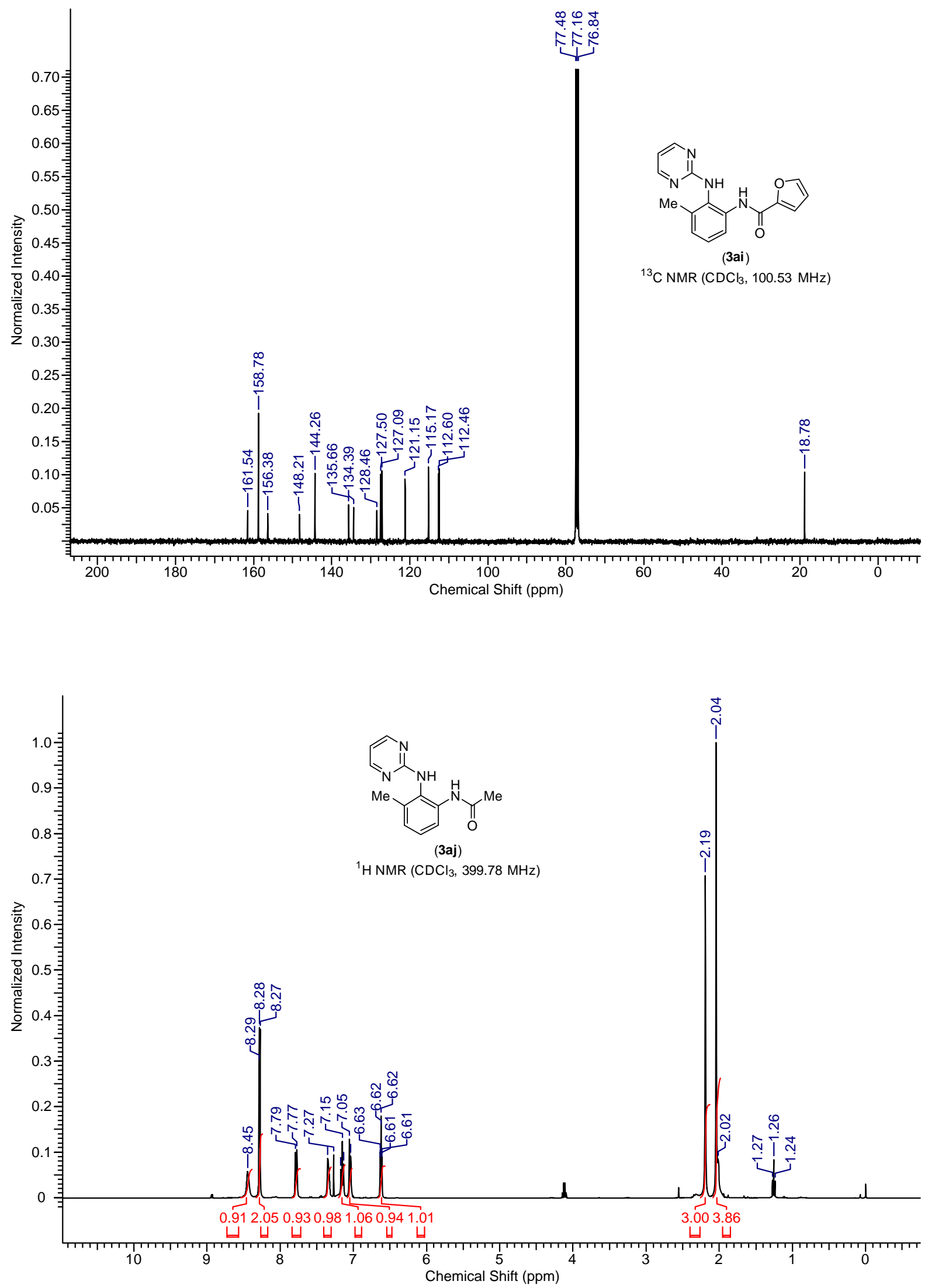

S91 

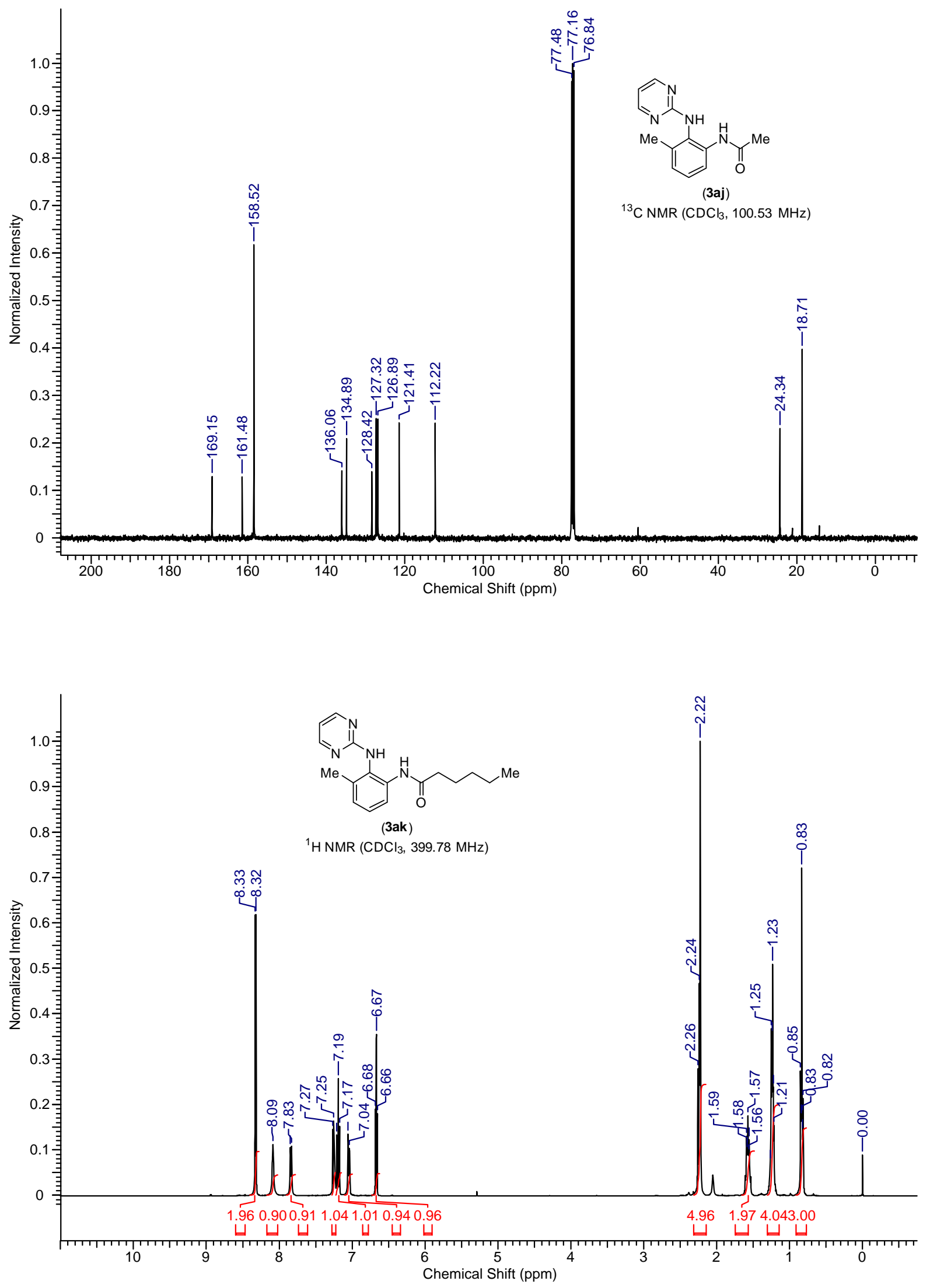

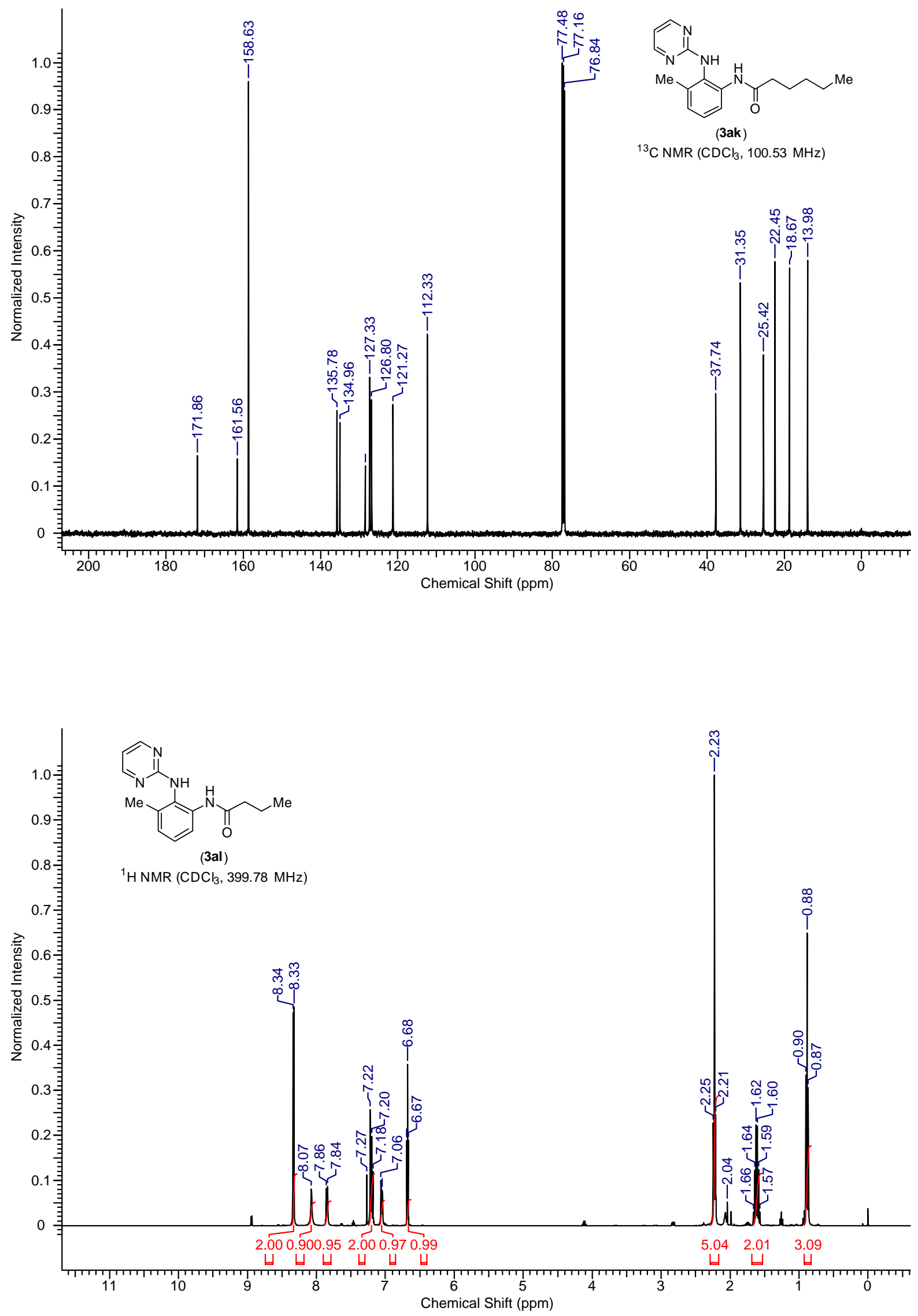

S93 

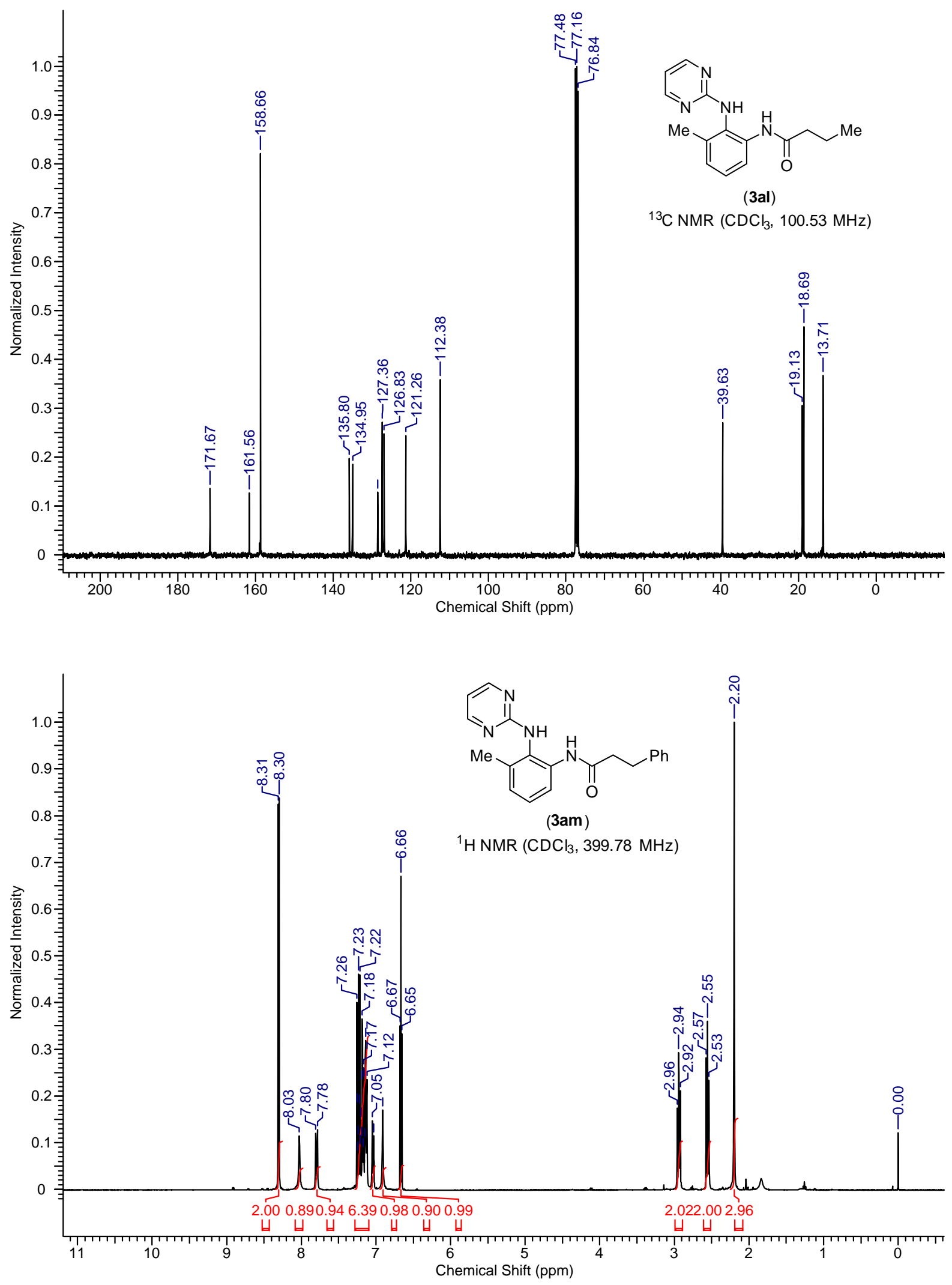

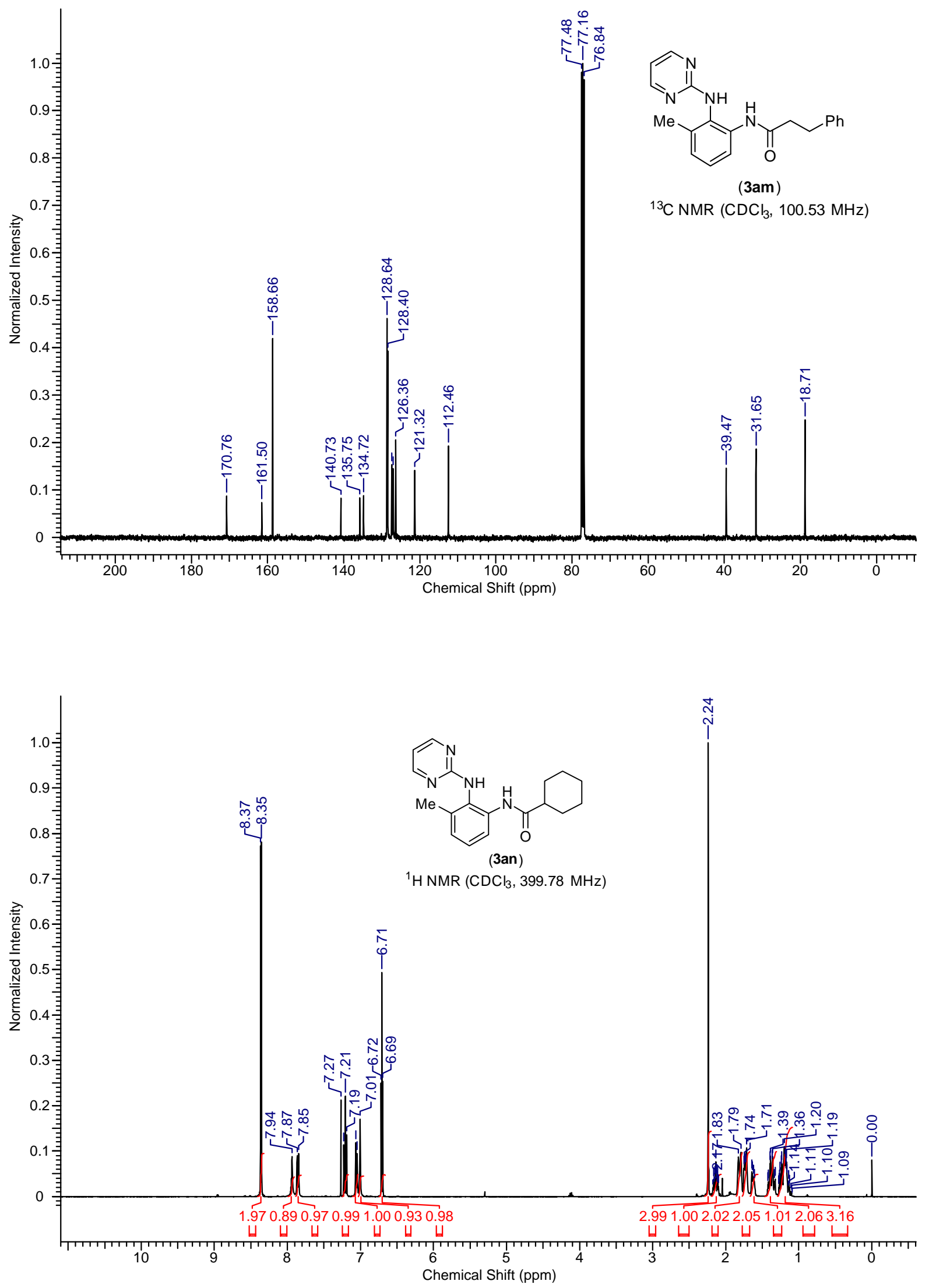


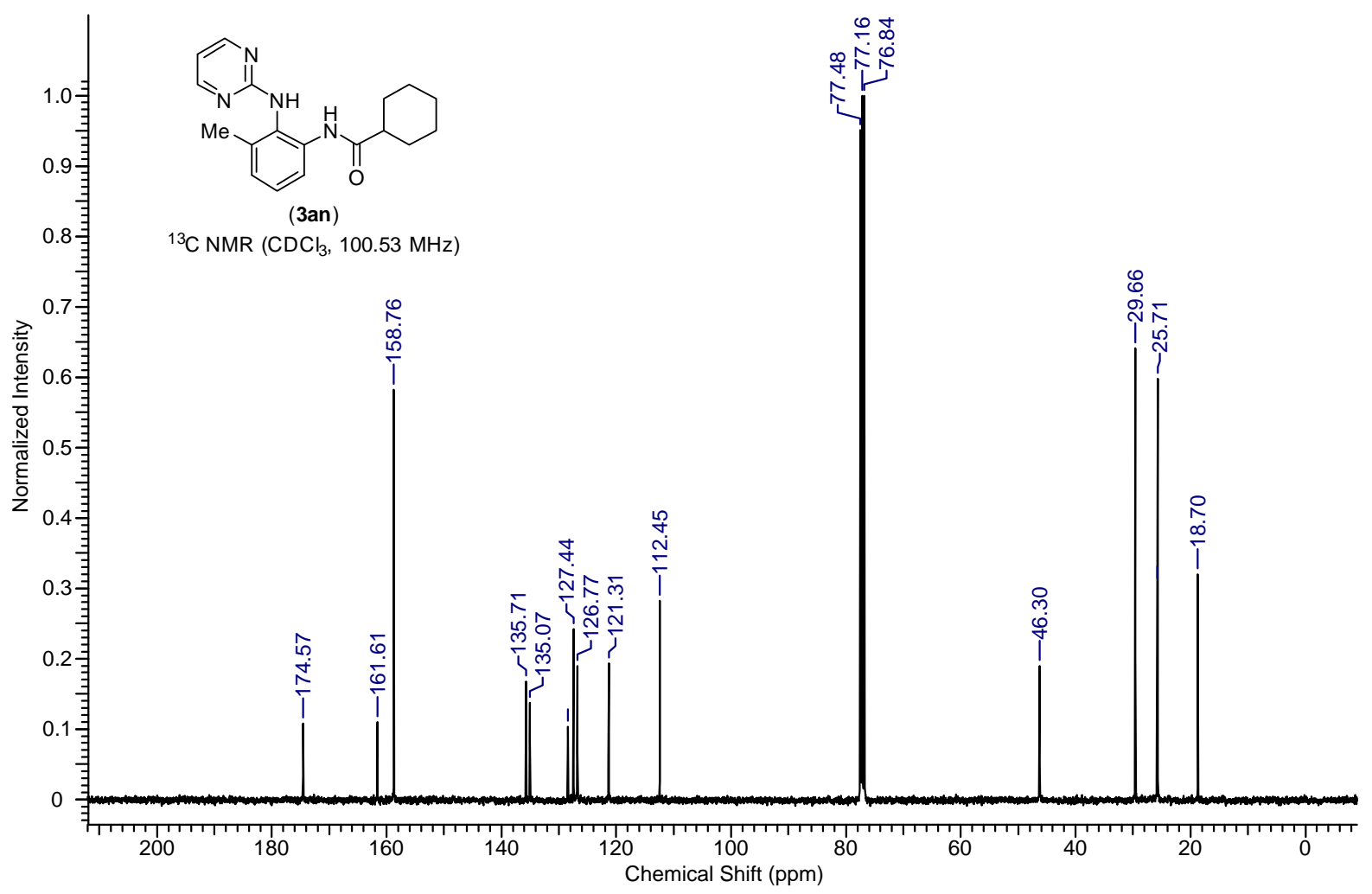

13.3) Spectra of Compound 5

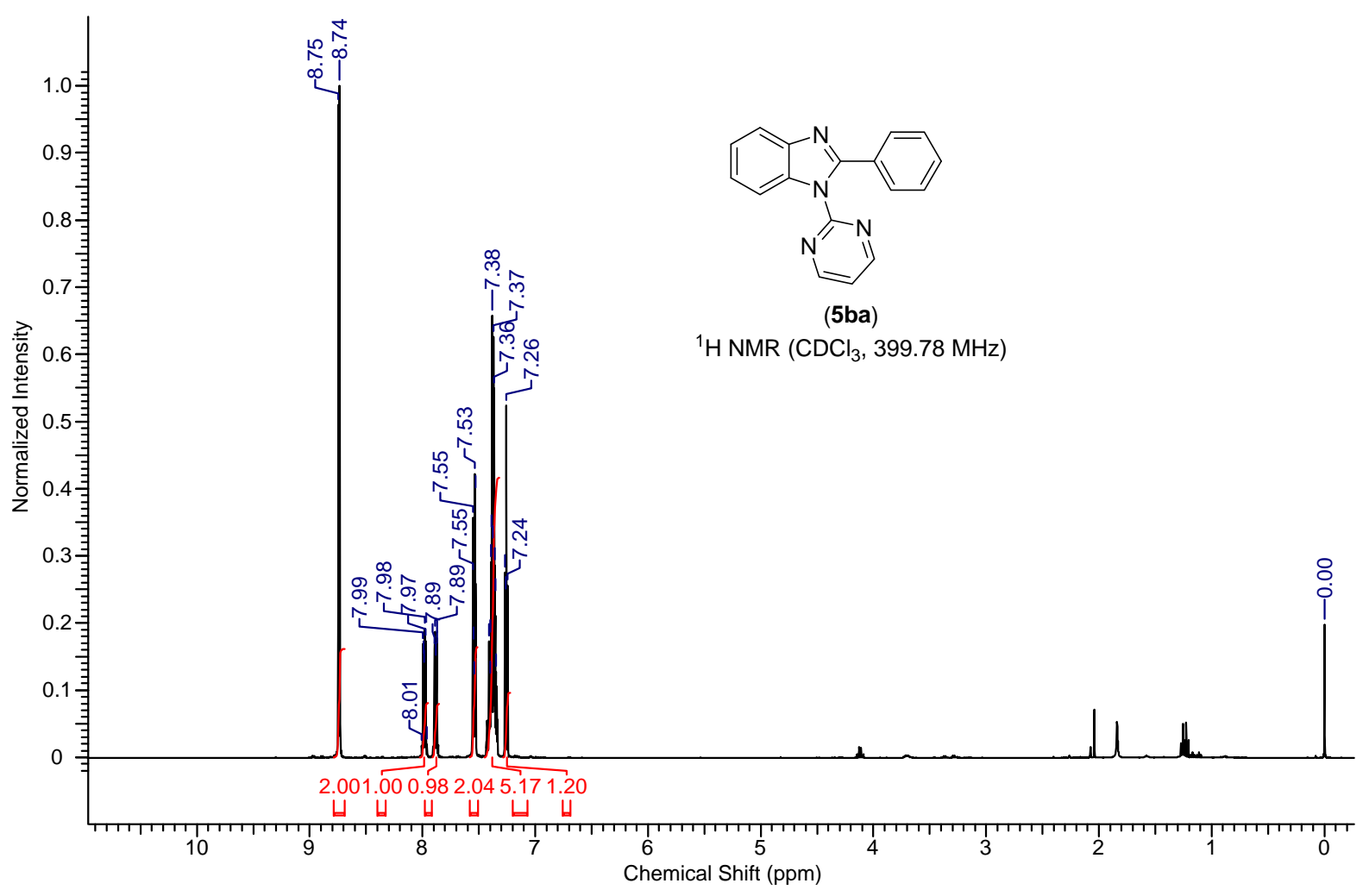



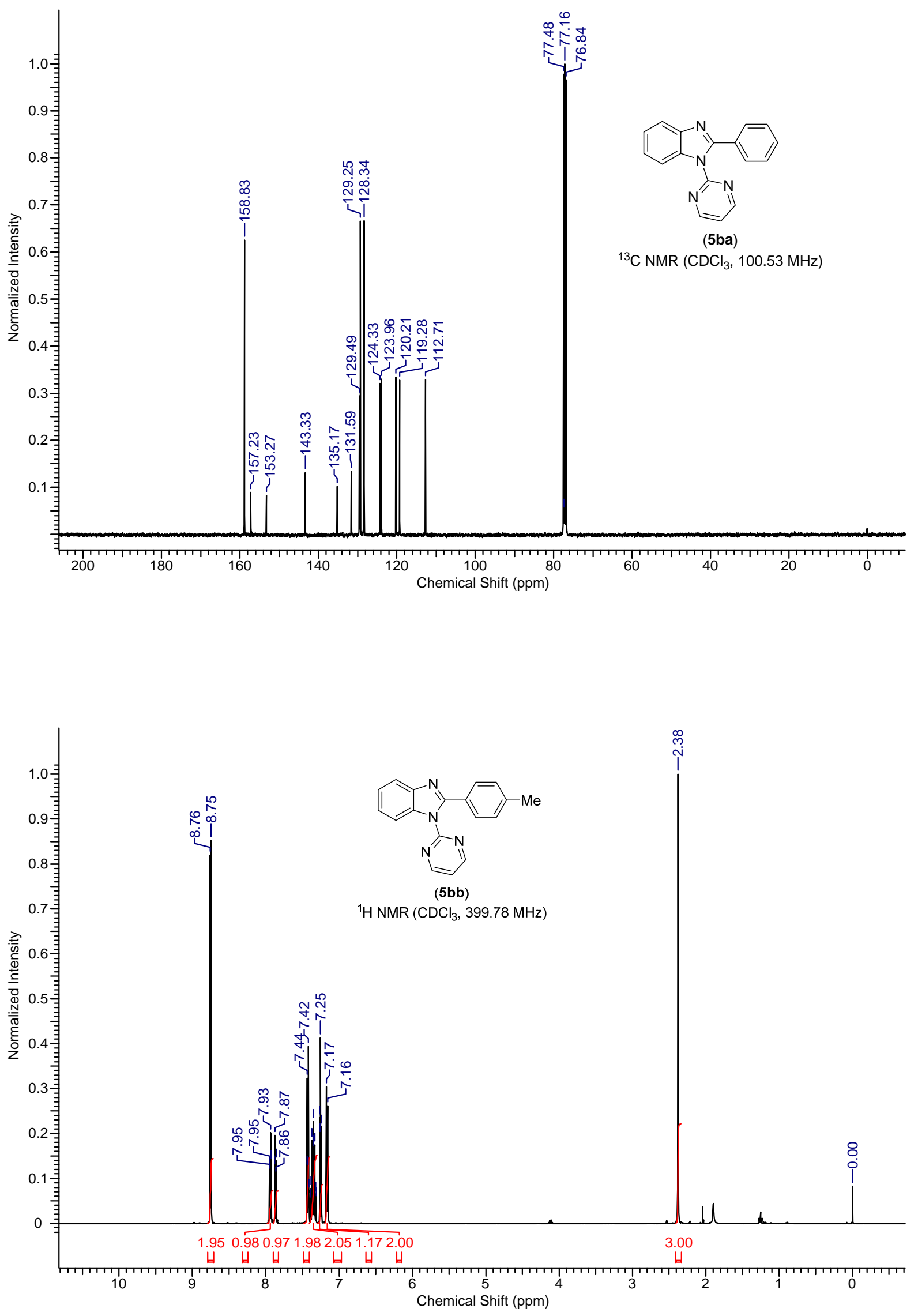

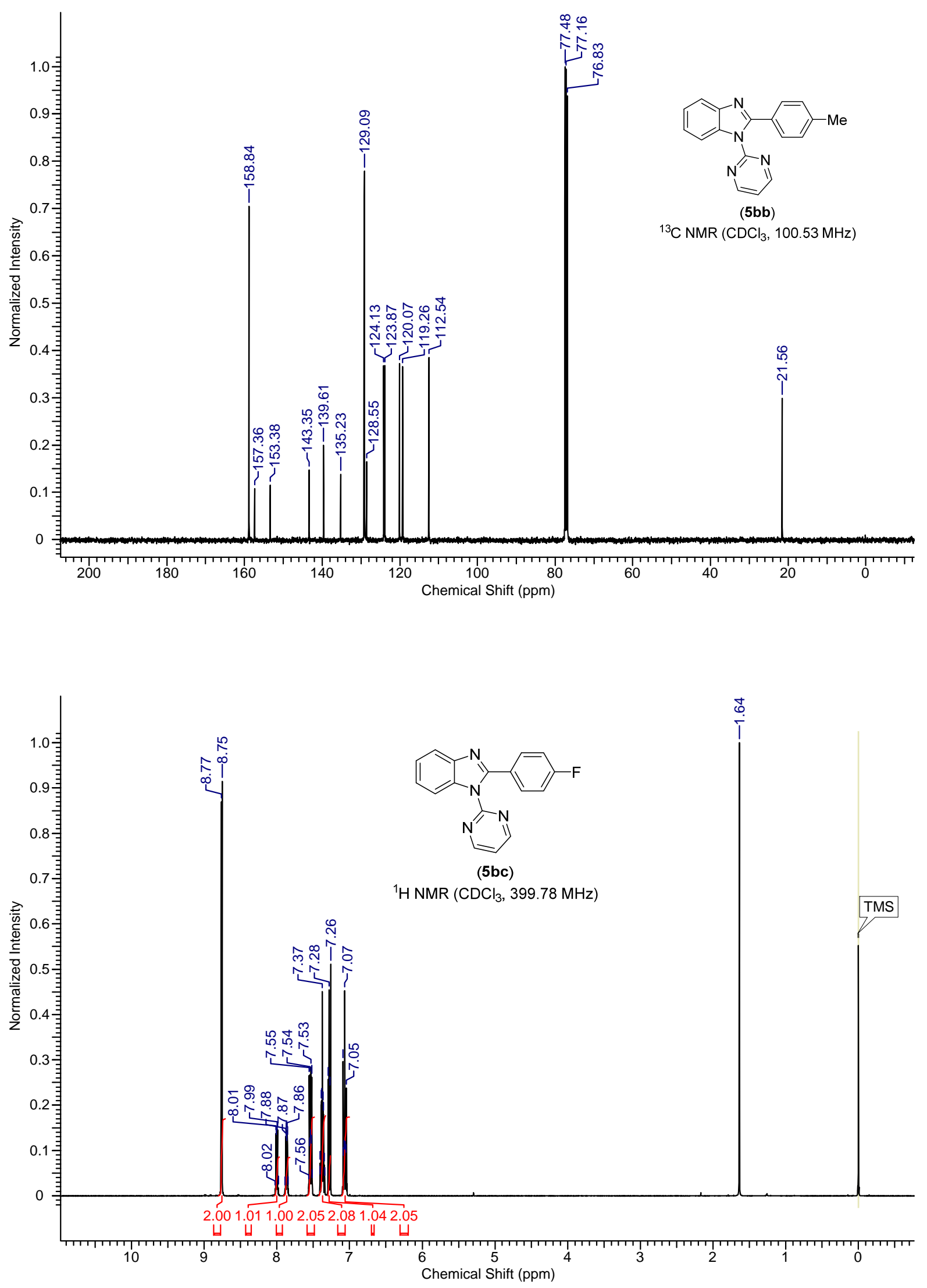

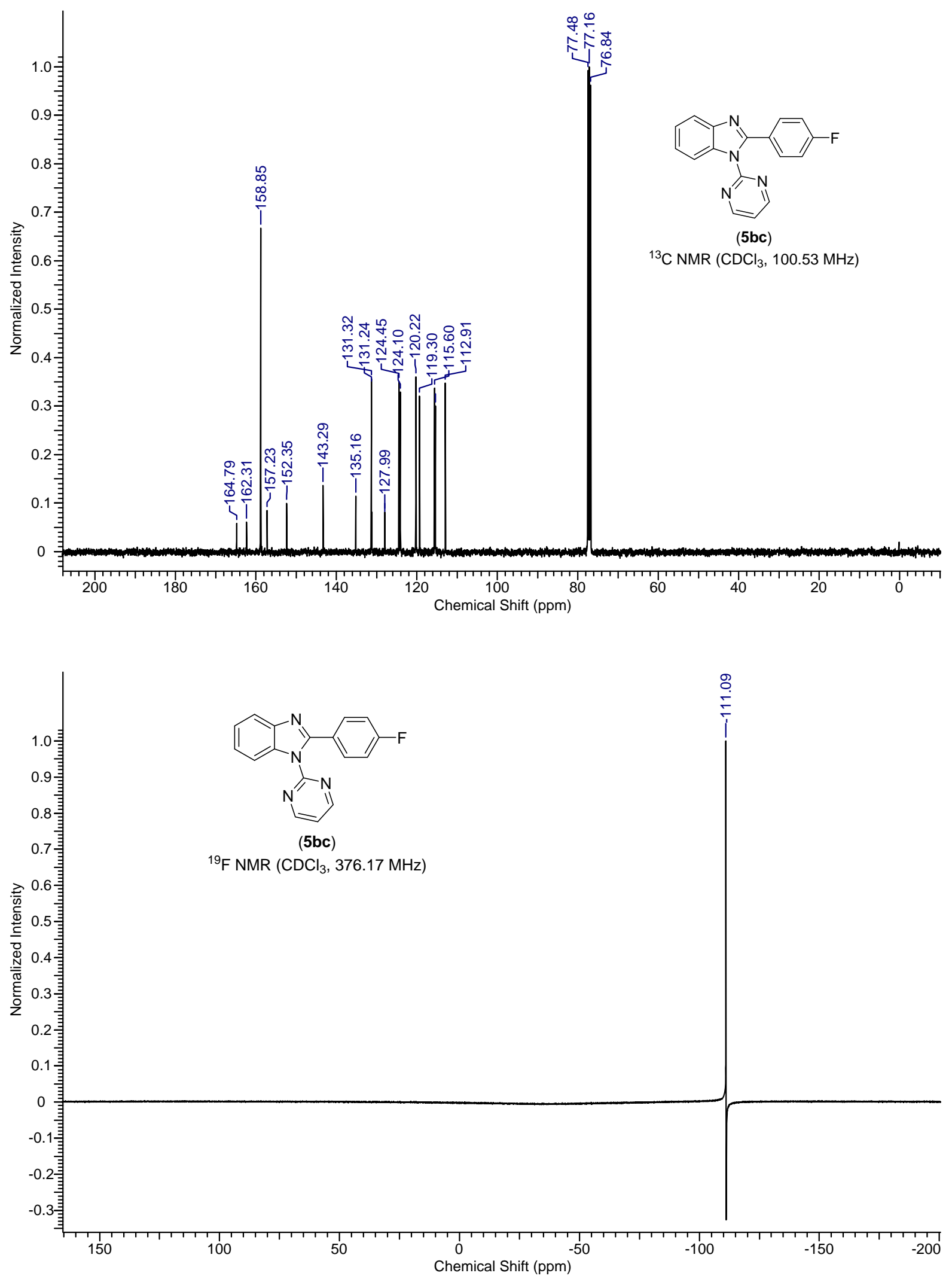

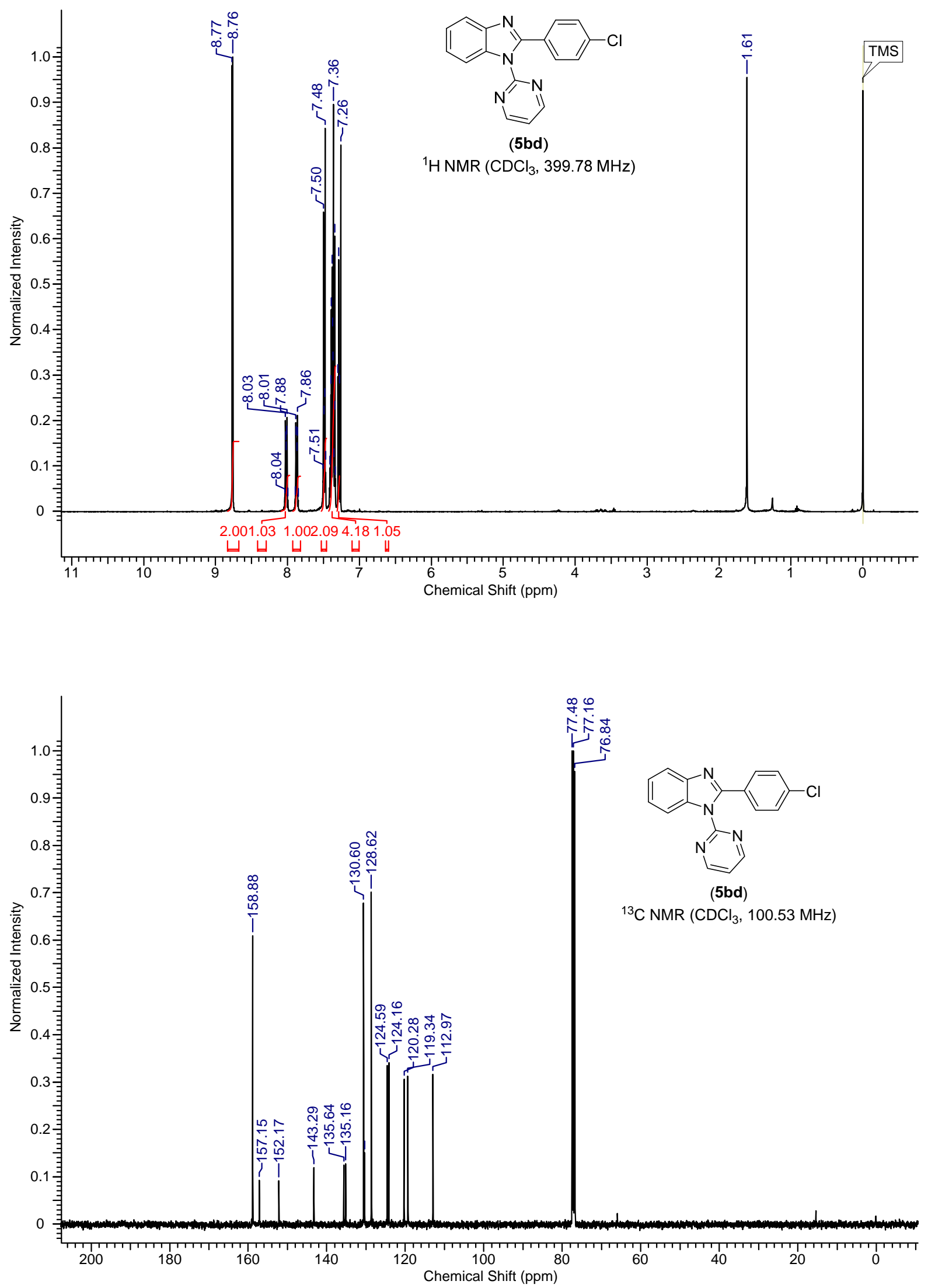

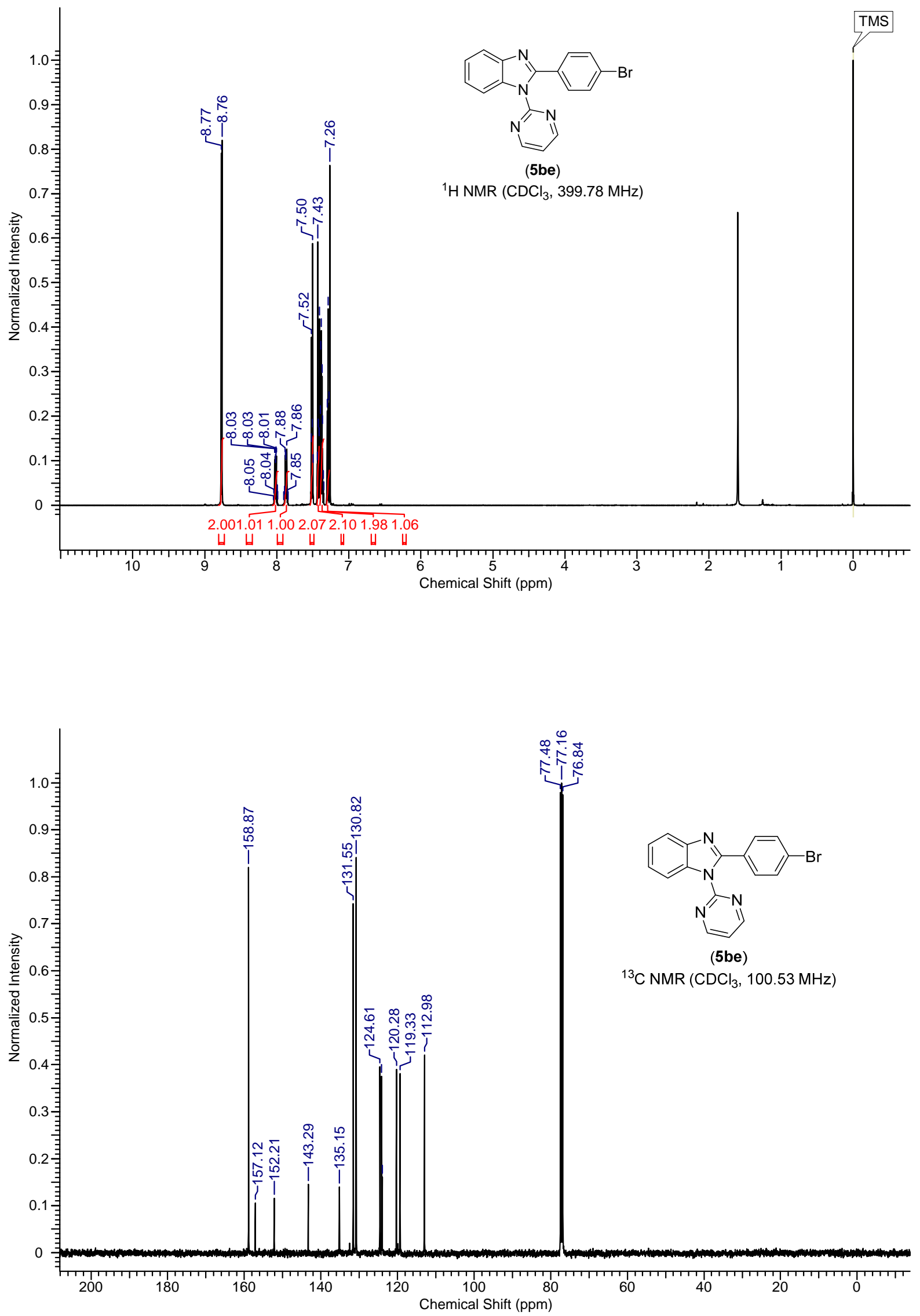

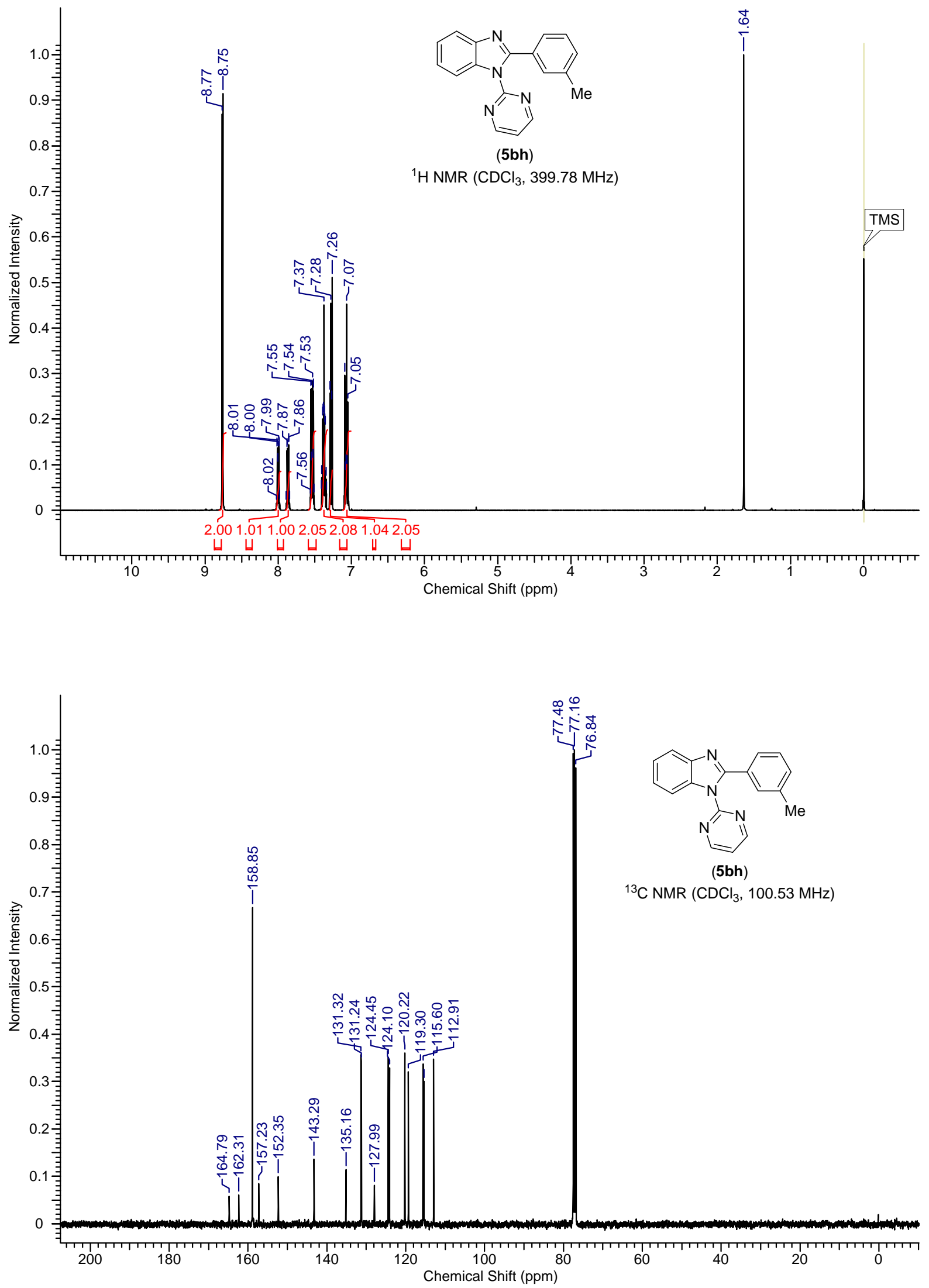

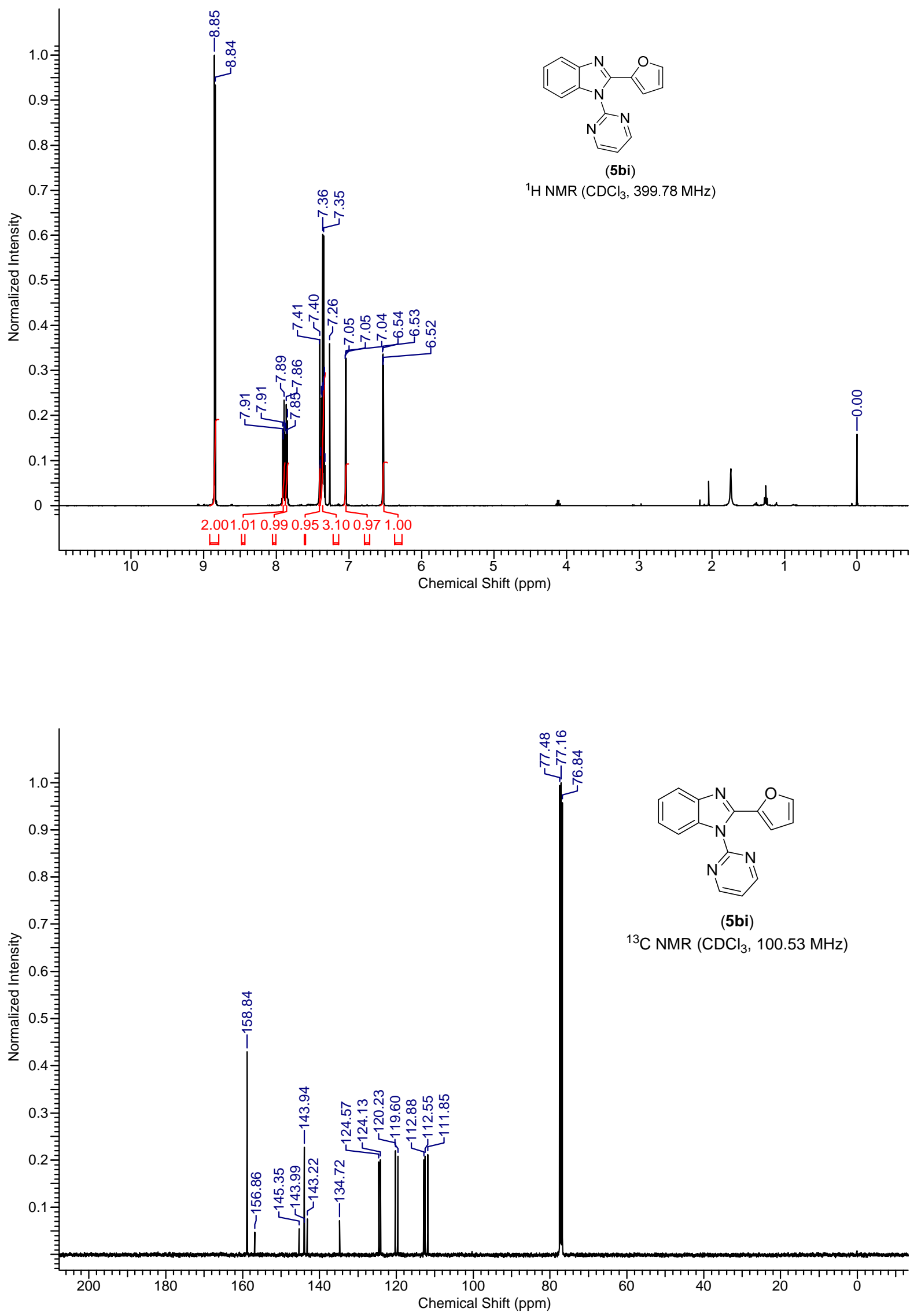

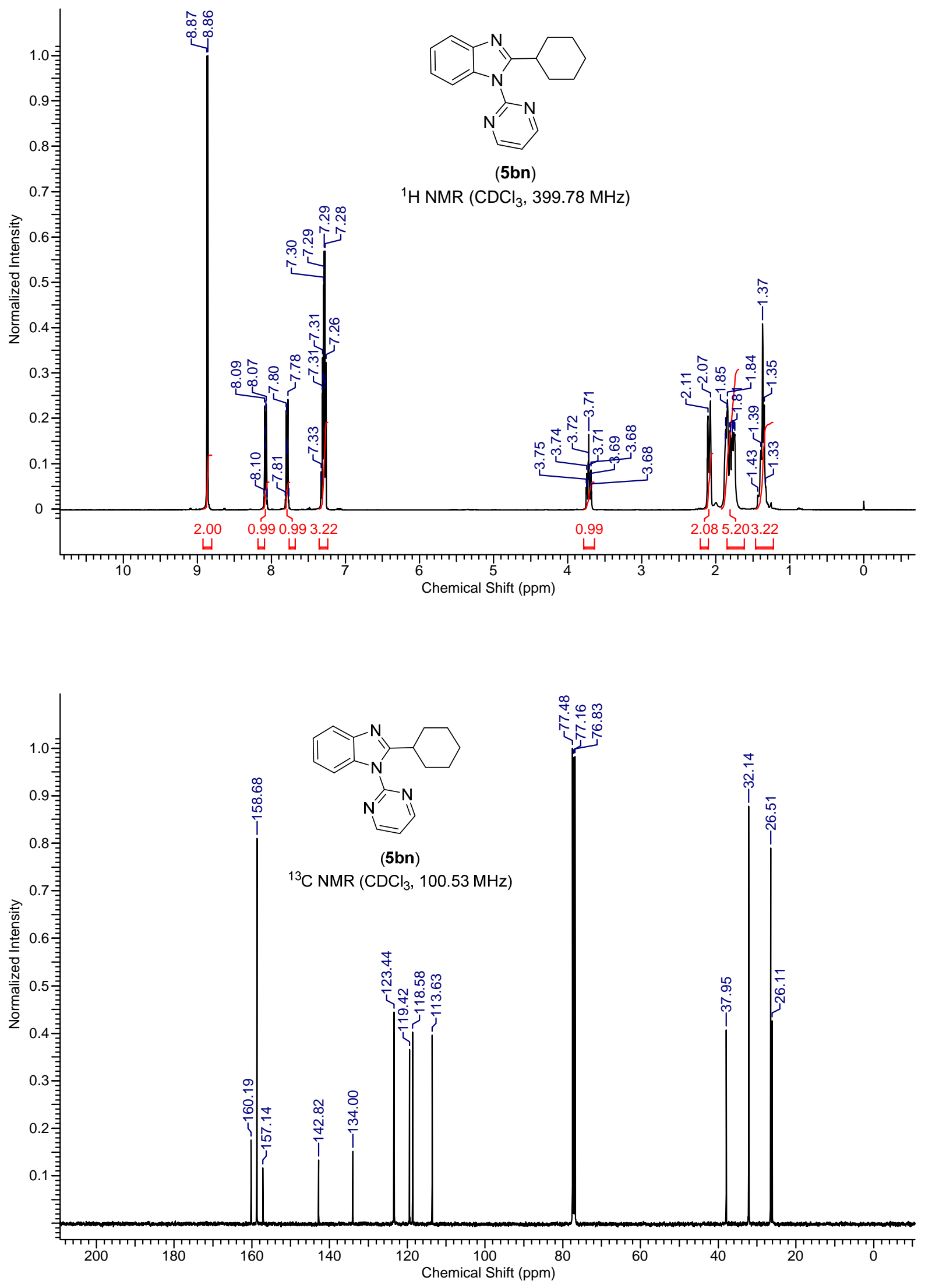


\section{4) Spectra of compound 6}
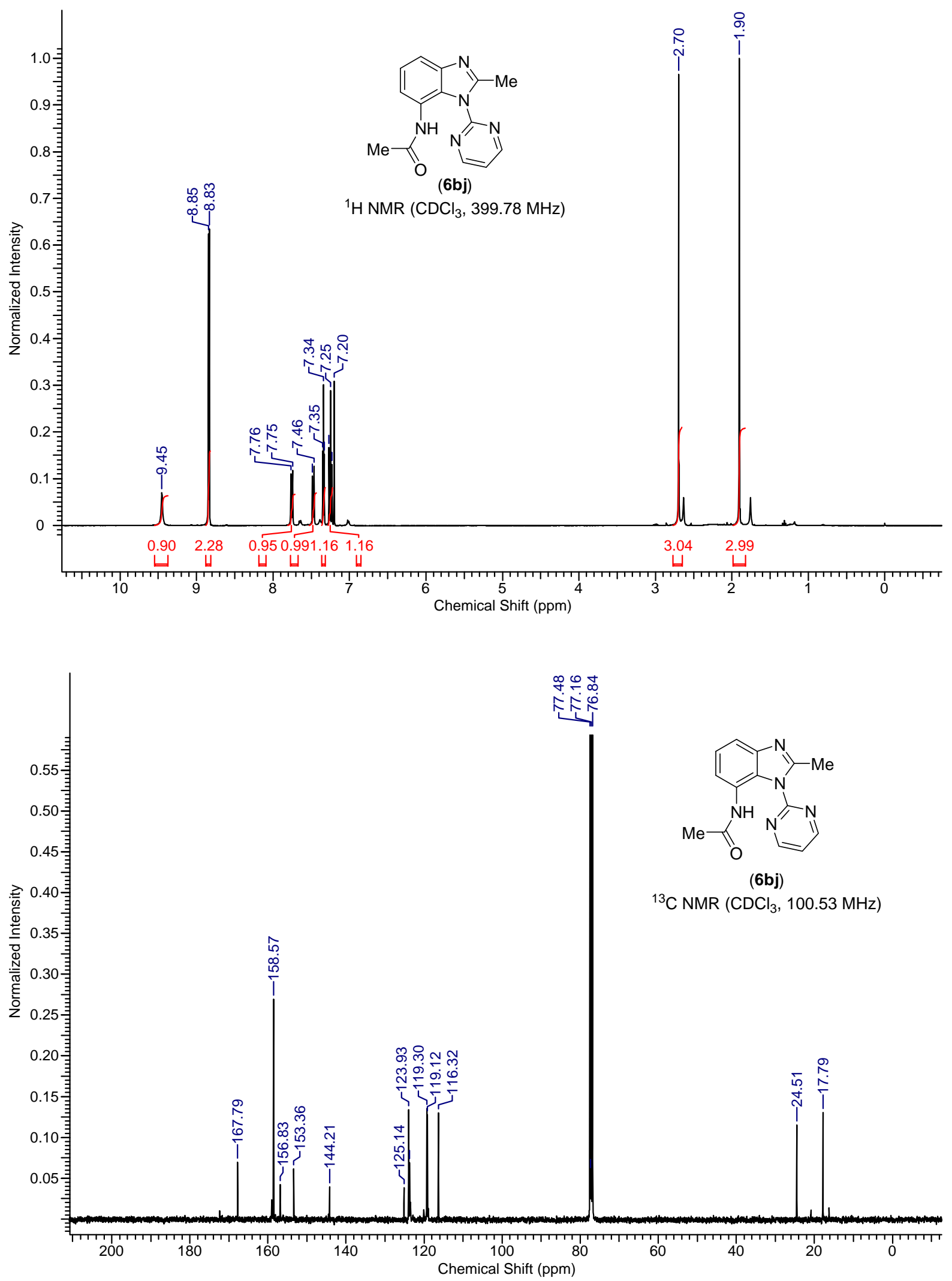

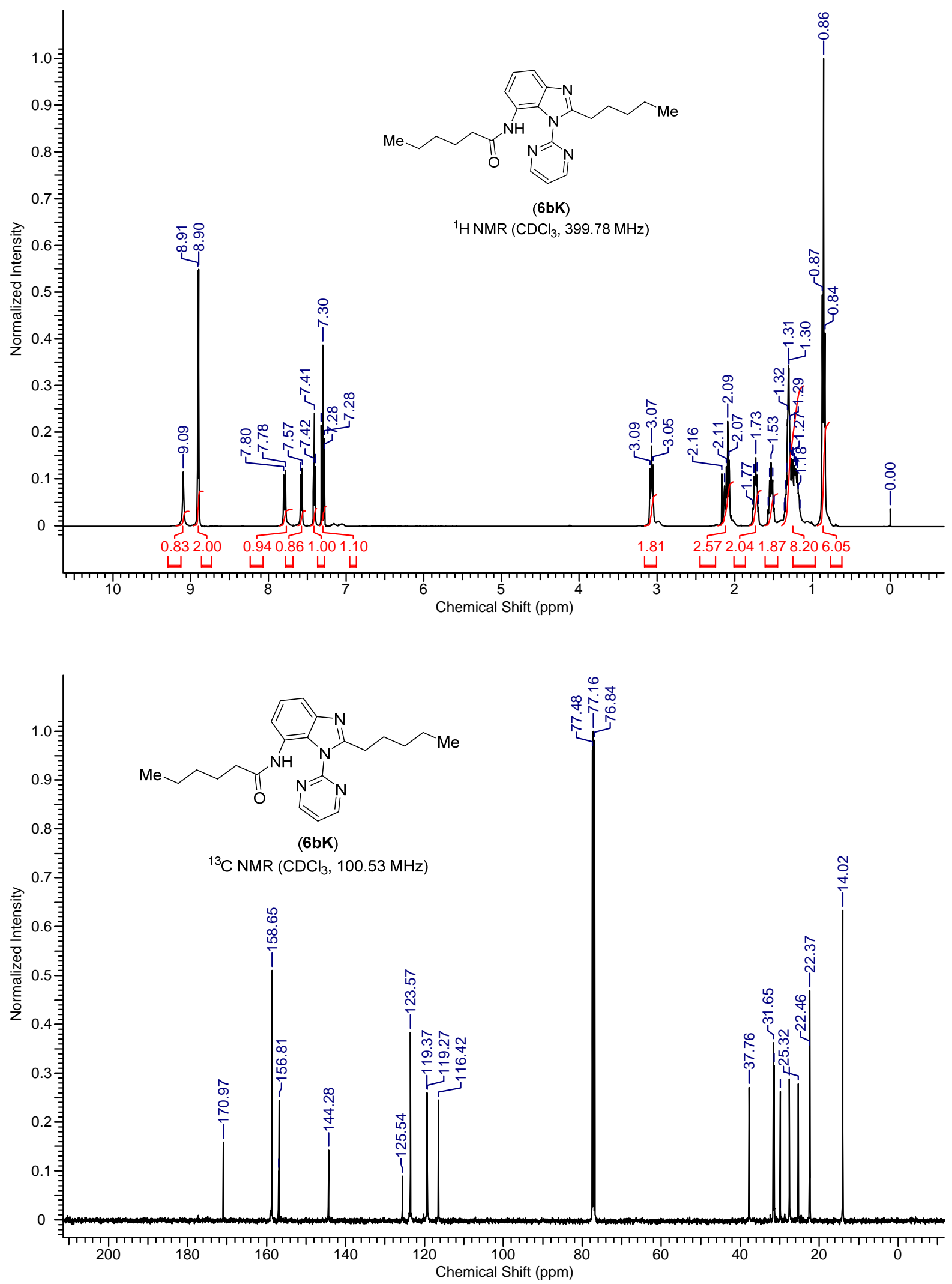

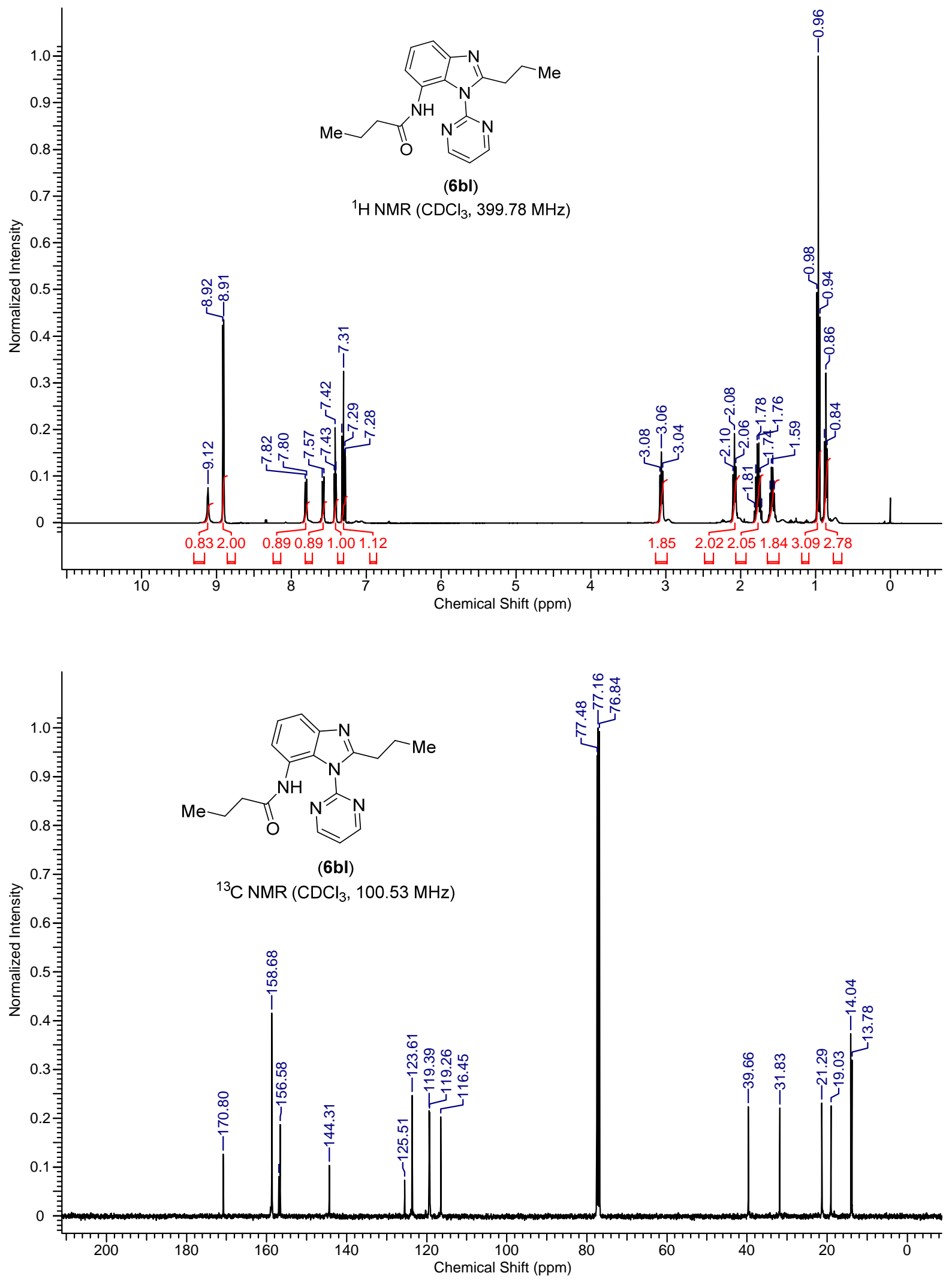

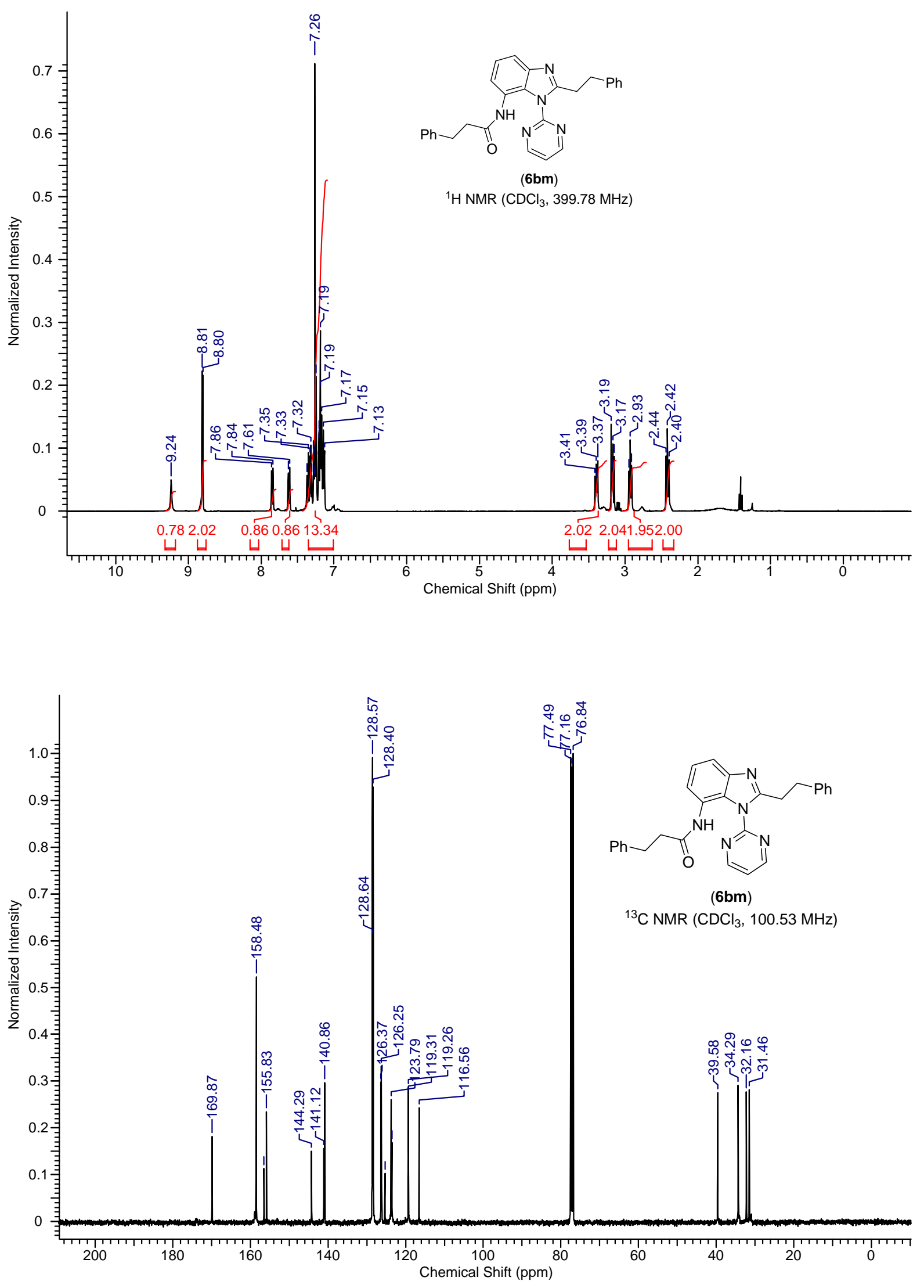
13.5) Spectra of compound 7
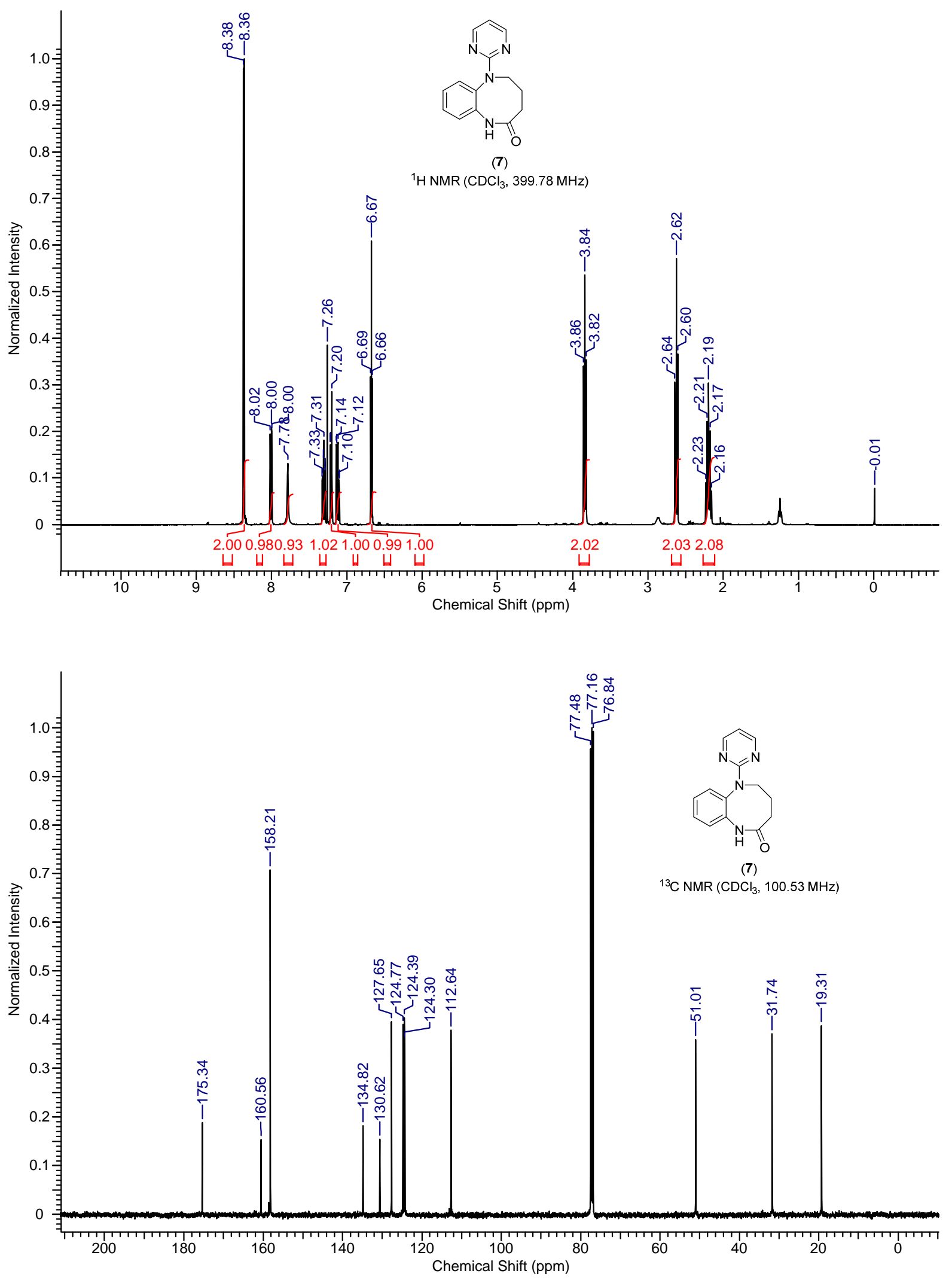


\section{6) Spectra of compound 8}
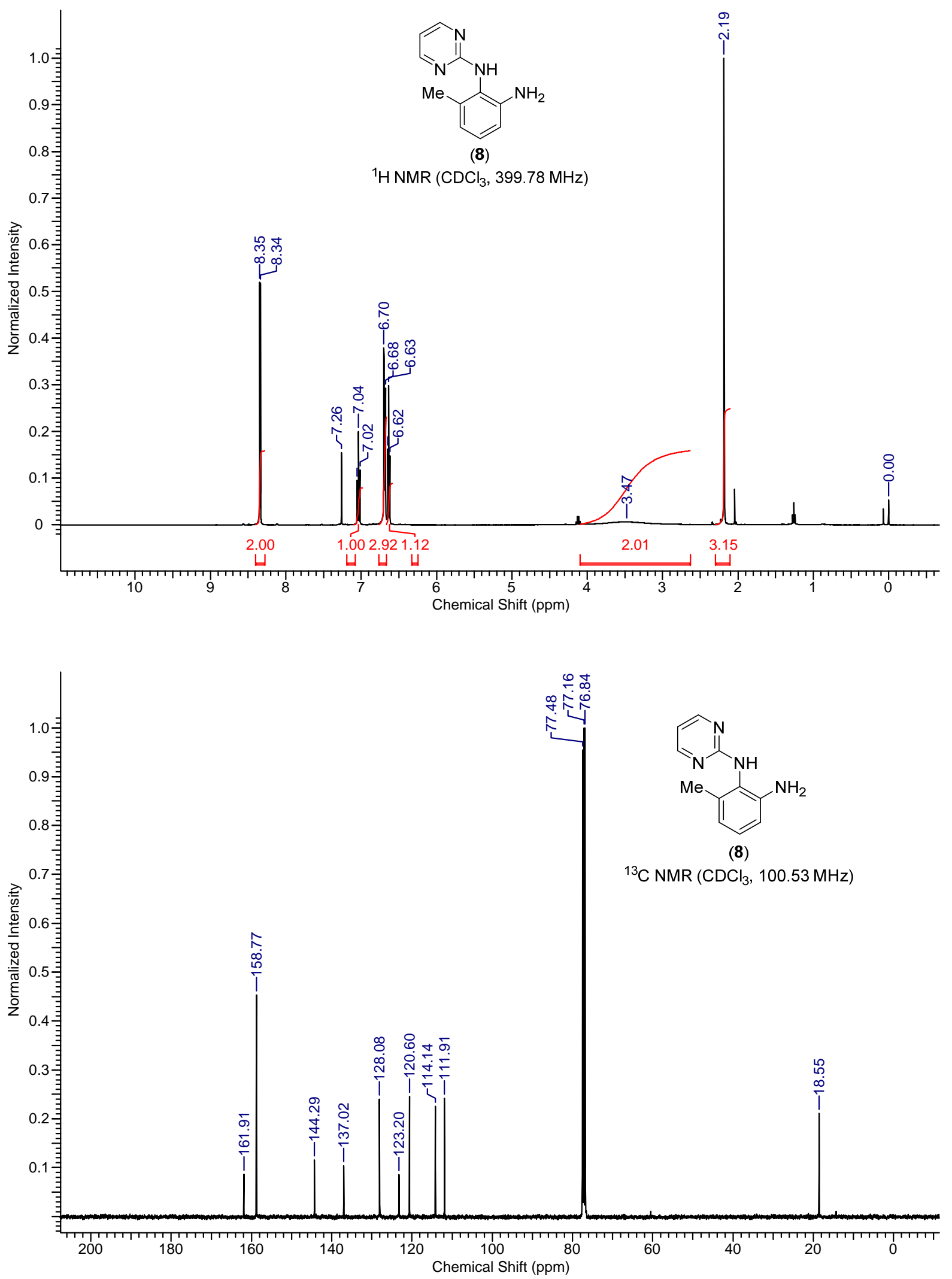
13.7) Spectra of compound 9
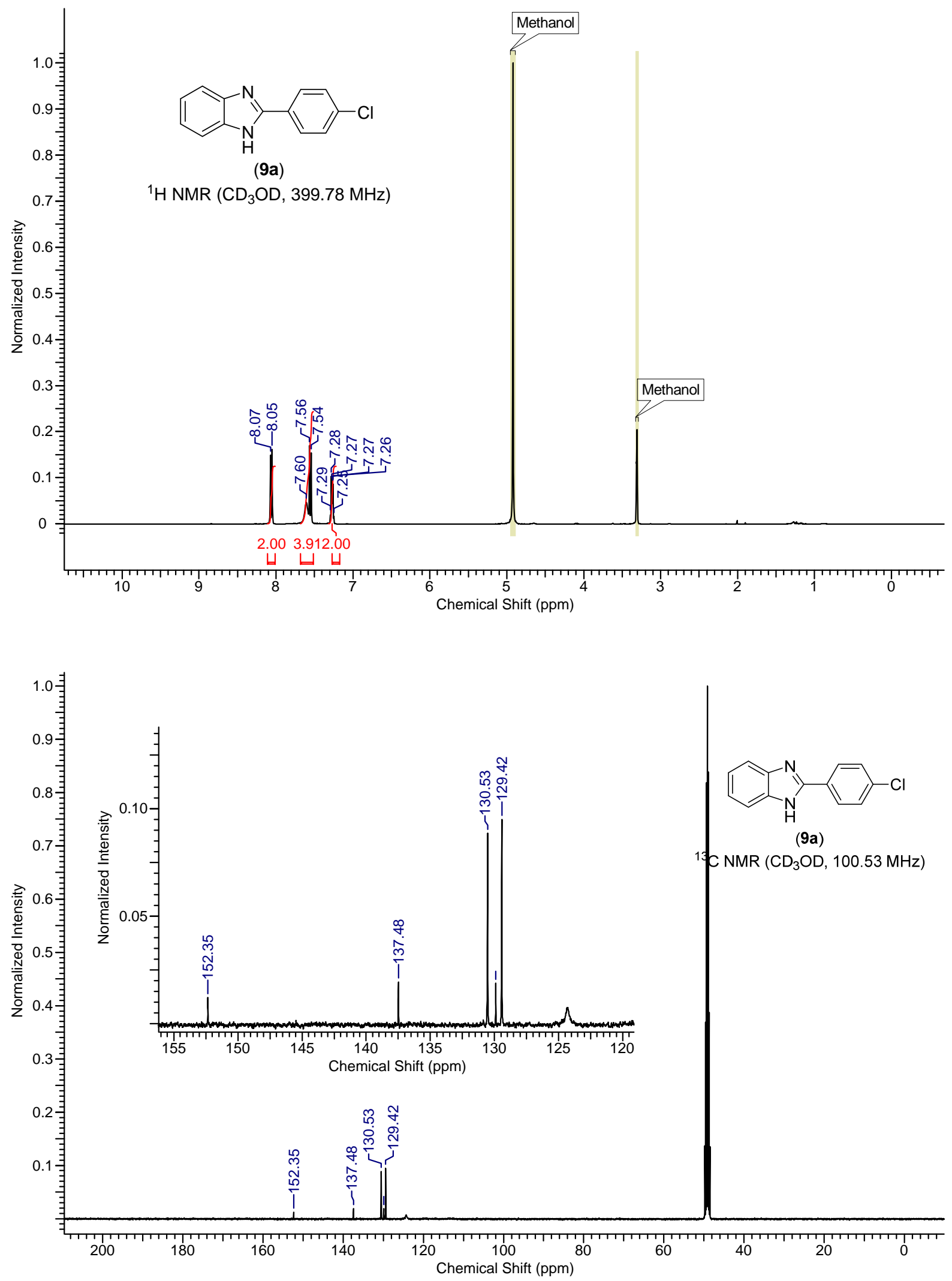

S111 

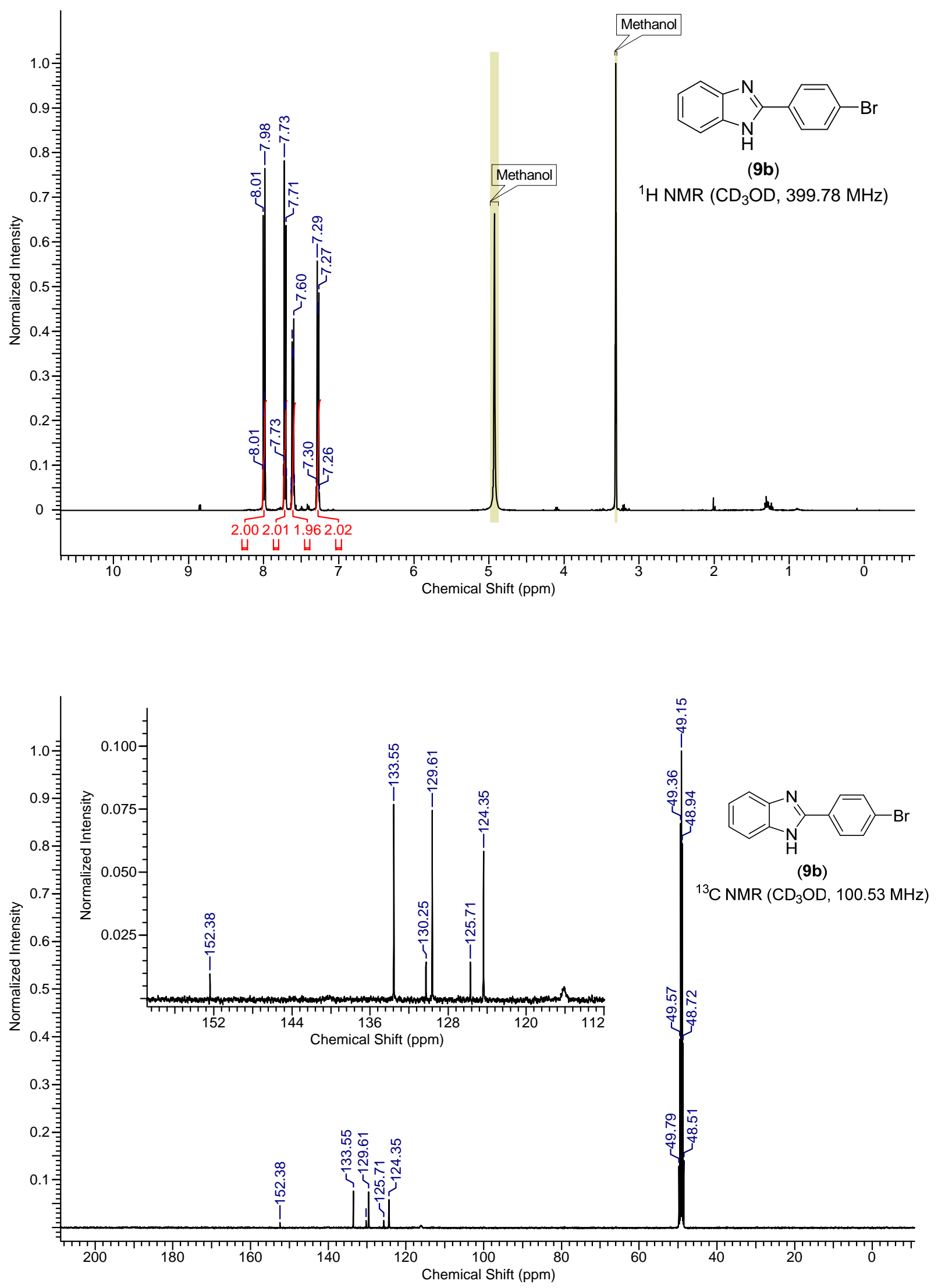

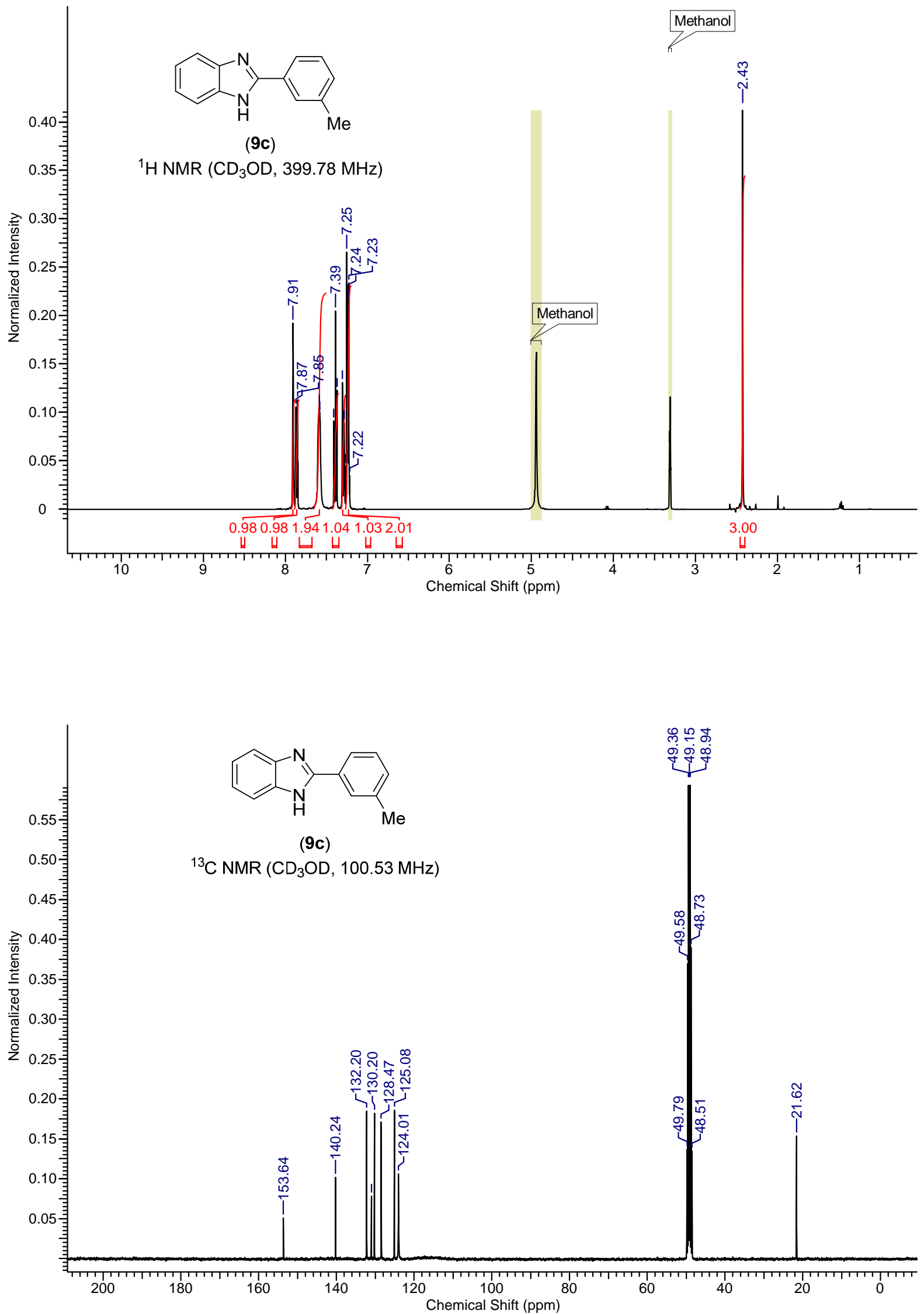


\section{8) Spectra of compound 10}
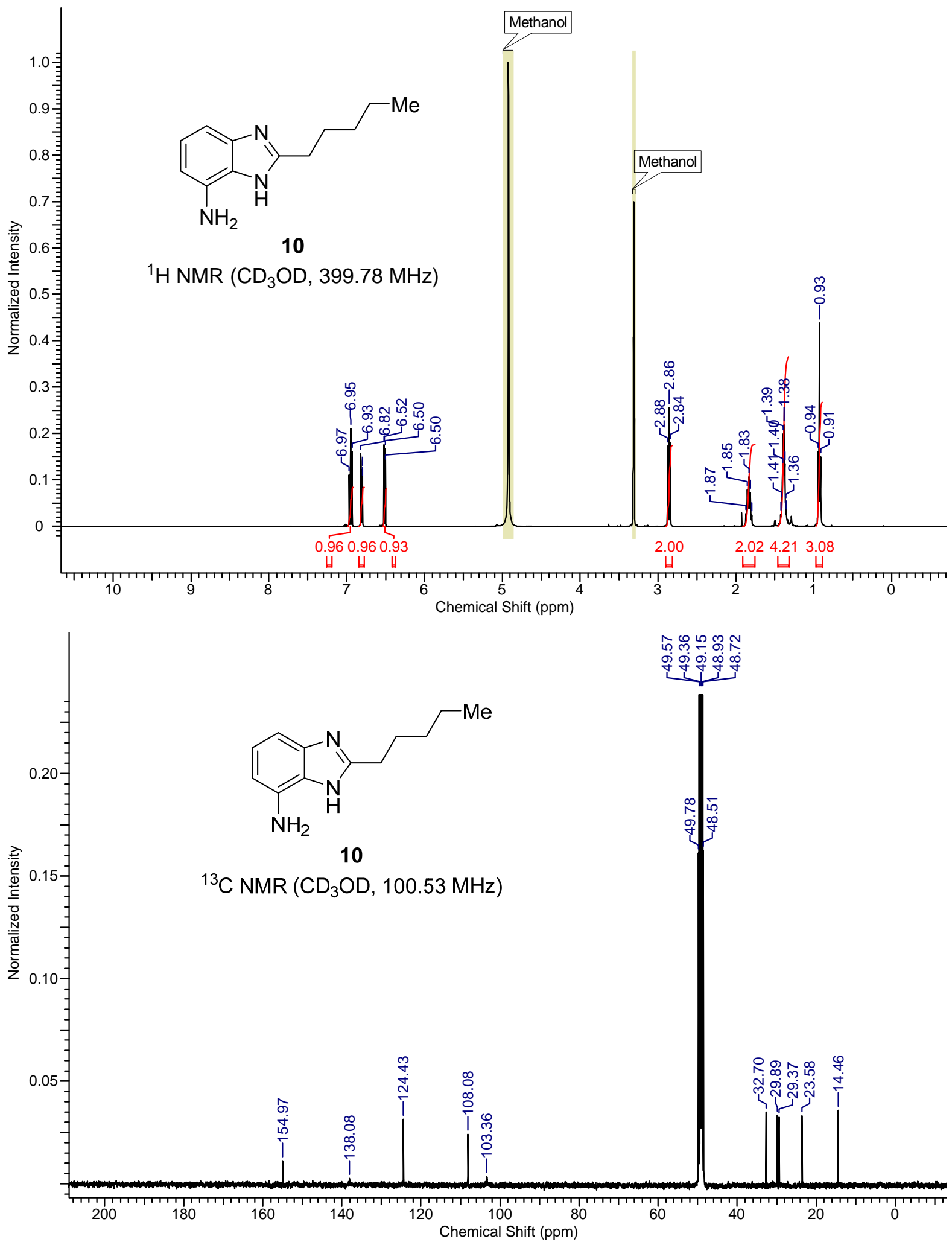